The hydrodynamics of swimming microorganisms

This article has been downloaded from IOPscience. Please scroll down to see the full text article.

2009 Rep. Prog. Phys. 72096601

(http://iopscience.iop.org/0034-4885/72/9/096601)

View the table of contents for this issue, or go to the journal homepage for more

Download details:

IP Address: 128.97.138.146

The article was downloaded on 17/04/2012 at 20:10

Please note that terms and conditions apply. 


\title{
The hydrodynamics of swimming microorganisms
}

\author{
Eric Lauga ${ }^{1}$ and Thomas R Powers ${ }^{2}$ \\ ${ }^{1}$ Department of Mechanical and Aerospace Engineering, University of California, San Diego, La Jolla, \\ CA 92093-0411, USA \\ 2 Division of Engineering, Brown University, Providence, RI 02912-9104, USA \\ E-mail: elauga@ucsd.edu and Thomas_Powers@brown.edu
}

Received 17 December 2008, in final form 5 May 2009

Published 25 August 2009

Online at stacks.iop.org/RoPP/72/096601

\begin{abstract}
Cell motility in viscous fluids is ubiquitous and affects many biological processes, including reproduction, infection and the marine life ecosystem. Here we review the biophysical and mechanical principles of locomotion at the small scales relevant to cell swimming, tens of micrometers and below. At this scale, inertia is unimportant and the Reynolds number is small. Our emphasis is on the simple physical picture and fundamental flow physics phenomena in this regime. We first give a brief overview of the mechanisms for swimming motility, and of the basic properties of flows at low Reynolds number, paying special attention to aspects most relevant for swimming such as resistance matrices for solid bodies, flow singularities and kinematic requirements for net translation. Then we review classical theoretical work on cell motility, in particular early calculations of swimming kinematics with prescribed stroke and the application of resistive force theory and slender-body theory to flagellar locomotion. After examining the physical means by which flagella are actuated, we outline areas of active research, including hydrodynamic interactions, biological locomotion in complex fluids, the design of small-scale artificial swimmers and the optimization of locomotion strategies.

(Some figures in this article are in colour only in the electronic version)

This article was invited by Christoph Schmidt.
\end{abstract}

\section{Contents}

1. Introduction

2. Overview of mechanisms of swimming motility

3. Flows at low Reynolds number

3.1. General properties

3.2. Motion of solid bodies

3.3. Flow singularities

4. Life at low Reynolds number

4.1. Reinterpreting the Reynolds number

4.2. The swimming problem

4.3. Drag-based thrust

4.4. The scallop theorem

5. Historical studies, and further developments

5.1. Taylor's swimming sheet

5.2. Local drag theory for slender rods

5.3. Slender-body theory
6. Physical actuation $\quad 15$

6.1. Boundary actuation $\quad 15$

6.2. Distributed actuation 16

7. Hydrodynamic interactions 18

7.1. Interactions between cells 18

7.2. Interactions between cells and boundaries 20

7.3. Interactions between flagella 22

8. Swimming in complex fluids 24

9. Artificial swimmers and optimization 26

9.1. Designing artificial swimmers 26

9.2. Exploiting low-Re locomotion 27

9.3. Optimization 27

10. Conclusion and outlook 29

Acknowledgments $\quad 29$

References $\quad 30$ 


\section{Introduction}

Our world is filled with swimming microorganisms: the spermatozoa that fuse with the ovum during fertilization, the bacteria that inhabit our guts, the protozoa in our ponds, the algae in the ocean; these are but a few examples of a wide biological spectrum. The reasons microorganisms move are familiar. Bacteria such as Escherichia coli detect gradients in nutrients and move to regions of higher concentration [1]. The spermatozoa of many organisms swim to the ovum, sometimes in challenging environments such as tidal pools in the case of sea urchins or cervical mucus in the case of humans [2]. Paramecium cells swim to evade predator rotifers.

What is perhaps less familiar is the fact that the physics governing swimming at the micrometer scale is different from the physics of swimming at the macroscopic scale. The world of microorganisms is the world of low 'Reynolds number', a world where inertia plays little role and viscous damping is paramount. As we describe below, the Reynolds number $R e$ is defined as $\operatorname{Re}=\rho U L / \eta$, where $\rho$ is the fluid density, $\eta$ is the viscosity and $U$ and $L$ are characteristic velocity and length scales of the flow, respectively. Swimming strategies employed by larger organisms that operate at high Reynolds number, such as fish, birds or insects [3-8], are ineffective at the small scale. For example, any attempt to move by imparting momentum to the fluid, as is done in paddling, will be foiled by the large viscous damping. Therefore microorganisms have evolved propulsion strategies that successfully overcome and exploit drag. The aim of this review is to explain the fundamental physics upon which these strategies rest.

The study of the physics of locomotion at low Reynolds number has a long history. In 1930, Ludwig [9] pointed out that a microorganism that waves rigid arms like oars is incapable of net motion. Over the years there have been many classic reviews, from the general perspective of animal locomotion [10], from the perspective of fluid dynamics at low Reynolds number [3,11-16], and from the perspective of the biophysics and biology of cell motility [1,17-21]. Nevertheless, the number of publications in the field has grown substantially in the past few years. This growth has been spurred in part by new experimental techniques for studying cell motility. Traditionally, motile cells have been passively observed and tracked using light microscopy. This approach has led to crucial insights such as the nature of the chemotaxis strategy of $E$. coli [1]. These techniques continue to improve and find applicability to a wide range of microorganisms [22]. Other recent advances in visualization techniques, such as the fluorescent staining of flagella [23] in living, swimming bacteria, also elucidate the mechanics of motility. A powerful new contribution is the ability to measure forces at the scale of single organisms and single motors. For example, it is now possible to measure the force required to hold a swimming spermatozoon [24-26], alga [27] or bacterium [28] in an optical trap. Atomic force microscopy also allows direct measurement of the force exerted by cilia [29]. Thus the relation between force and the motion of the flagellum can be directly assessed. These measurements of force allow new approaches to biological questions, such the heterogeneity of motor behavior in genetically identical bacteria. Measurements of force together with quantitative observation of cell motion motivate the development of detailed hydrodynamic theories that can constrain or rule out models of cell motion.

The goal of this review is to describe the theoretical framework for locomotion at low Reynolds number. Our focus is on analytical results, but our aim is to emphasize physical intuition. In section 2 , we give some examples of how microorganisms swim. After a brief general review of low Reynolds number hydrodynamics (section 3), we outline the fundamental properties of locomotion without inertia (section 4). We then discuss the classic contributions of Taylor [30], Hancock [31] and Gray [32], who all but started the field more than 50 years ago (section 5); we also outline many of the subsequent works that followed. We proceed by introducing the different ways to physically actuate a flagella-based swimmer (section 6). We then move on to introduce topics of active research. These areas include the role of hydrodynamic interactions, such as the interactions between two swimmers, or between a wall and a swimmer (section 7); locomotion in non-Newtonian fluids such as the mucus of the female mammalian reproductive tract (section 8) and the design of artificial swimmers and the optimization of locomotion strategies in an environment at low Reynolds number (section 9). Our coverage of these topics is motivated by intellectual curiosity and the desire to understand the fundamental physics of swimming; the relevance of swimming in biological processes such as reproduction or bacterial infection; and the practical desire to build artificial swimmers, pumps and transporters in microfluidic systems.

Our review is necessarily limited to a small cross-section of current research. There are many closely related aspects of 'life at low Reynolds number' that we do not address, such as nutrient uptake or quorum sensing; instead we focus on flow physics. Our hope is to capture some of the current excitement in this research area, which lies at the intersection of physics, mechanics, biology and applied mathematics, and is driven by clever experiments that shed a new light on the hidden world of microorganisms. Given the interdisciplinary nature of the subject, we have tried to make the review a self-contained starting point for the interested student or scientist.

\section{Overview of mechanisms of swimming motility}

In this section we motivate our review with a short overview of mechanisms for swimming motility. We define a 'swimmer' to be a creature or object that moves by deforming its body in a periodic way. To keep the scope of the paper manageable, we do not consider other mechanisms that could reasonably be termed 'swimming', such as the polymerization of the actin of a host cell by pathogens of the genus Listeria [33], or the gasvesicle mediated buoyancy of aquatic micoorganisms such as Cyanobacteria [34].

Many microscopic swimmers use one or more appendages for propulsion. The appendage could be a relatively stiff helix that is rotated by a motor embedded in the cell wall, as in the case of E. coli [35] (figure 1(a)), or it could be a flexible filament undergoing whip-like motions due to the action of molecular 


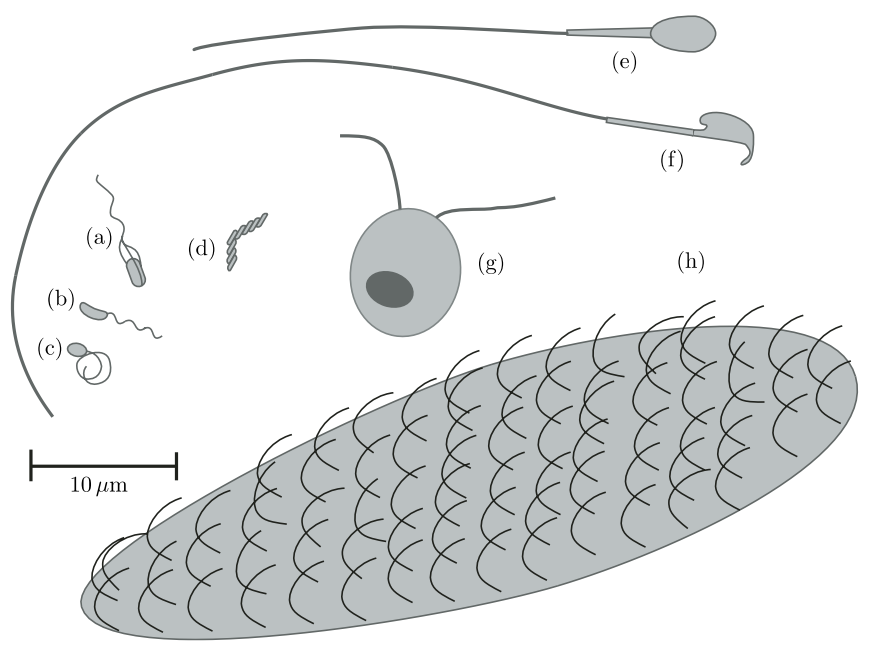

Figure 1. Sketches of microscopic swimmers, to scale. (a) E. coli. (b) C. crescentus. (c) R. sphaeroides, with flagellar filament in the coiled state. (d) Spiroplasma, with a single kink separating regions of right-handed and left-handed coiling. (e) Human spermatozoon. (f) Mouse spermatozoon. (g) Chlamydomonas. (h) A smallish Paramecium.

motors distributed along the length of the filament, as in the sperm of many species [21] (figures 1(e) and (f)). For example, the organelle of motility in E. coli and Salmonella typhimurium is the bacterial flagellum, consisting of a rotary motor [36], a helical filament, and a hook which connects the motor to the filament $[1,20,37]$. The filament has a diameter of $\approx 20 \mathrm{~nm}$ and traces out a helix with contour length $\approx 10 \mu \mathrm{m}$. In the absence of external forces and moments, the helix is left-handed with a pitch $\approx 2.5 \mu \mathrm{m}$ and a helical diameter $\approx 0.5 \mu \mathrm{m}$ [23]. There are usually several flagella per cell. When the motor turns counter-clockwise (when viewed from outside the cell body), the filaments wrap into a bundle that pushes the cell along at speeds of $25-35 \mu \mathrm{m} \mathrm{s}^{-1}$ (see section 7.3.2) [38]. When one or more of the motors reverse, the corresponding filaments leave the bundle and undergo 'polymorphic' transformations in which the handedness of the helix changes; these polymorphic transformations can change the swimming direction of the cell [23].

There are many variations on these basic elements among swimming bacteria. For example, Caulobacter crescentus has a single right-handed helical filament (figure 1(b)), driven by a rotary motor that can turn in either direction. The motor preferentially turns clockwise, turning the filament in the sense to push the body forward [39]. During counterclockwise rotation the filament pulls the body instead of pushing. The motor of the bacterium Rhodobacter sphaeroides turns in only one direction but stops from time to time [40]. The flagellar filament forms a compact coil when the motor is stopped (figure 1(c)), and extends into a helical shape when the motor turns. Several archaea also use rotating flagella to swim, although far less is known about the archaea compared with bacteria. Archaea such as the various species of Halobacterium swim more slowly than bacteria, with typical speeds of $2-3 \mu \mathrm{m} \mathrm{s}^{-1}$ [41]. Although archaeal flagella also have a structure comprised of motor, hook and filament, molecular analysis of the constituent proteins shows that archaeal and bacterial flagella are unrelated (see [42] and references therein).

There are also bacteria that swim with no external flagellar filaments. The flagella of spirochetes lie in the thin periplasmic space between the inner and outer cell membranes [43]. The flagellar motors are embedded in the cell wall at both poles of the elongated body of the spirochete, and the flagellar filaments emerge from the motor and wrap around the body. Depending on the species, there may be one or many filaments emerging from each end of the body. In some cases, such as the Lyme disease spirochete Borrelia burgdorferi, the body of the spirochete is observed to deform as it swims, and it is thought that the rotation of the periplasmic flagella causes this deformation which in turn leads to propulsion $[44,45]$. The deformation can be helical or planar. These bacteria swim faster in gel-like viscous environments than bacteria with external flagella [46,47]. Other spirochetes, such as Treponema primitia, do not change shape at all as they swim, and it is thought that motility develops due to rotation of the outer membrane and cytoplasmic membrane in opposite senses [44, 48]. Finally, we mention the case of Spiroplasma, helically shaped bacteria with no flagella (figure 1(d)). These cells swim via the propagation of pairs of kinks along the length of the body [49]. Instead of periplasmic flagella, the kinks are thought to be generated by contraction of the cytoskeleton [50-52].

Eukaryotic flagella and cilia are much larger than bacterial flagella, with a typical diameter of $\approx 200 \mathrm{~nm}$, and with an intricate internal structure [21]. The most common structure has nine microtubule doublets spaced around the circumference and running along the length of a flagellum or cilium, with two microtubules along the center. Molecular motors (dynein) between the doublets slide them back and forth, leading to bending deformations that propagate along the flagellum. There is a vast diversity in the beat pattern and length of eukaryotic flagella and cilia. For example, the sperm of many organisms consists of a head containing the genetic material propelled by a filament with a planar or even helical beat pattern, depending on the species [53]. The length of the flagellum is $12 \mu \mathrm{m}$ in some Hymenoptera [54], $\approx 20 \mu \mathrm{m}$ for hippos, $\approx 40 \mu \mathrm{m}$ for humans [2] (figure 1(e)), $\approx 80 \mu \mathrm{m}$ for mice (figure 1(f)), and can be $1 \mathrm{~mm}$ [55] or even several centimeters long in some fruit flies [56] (although in the last case the flagella are rolled up into pellets and offered to the female via a 'peashooter' effect [56]).

Many organisms have multiple flagella. Chlamydomonas reinhardtii is an alga with two flagella that can exhibit both ciliary and flagellar beat patterns (figure $1(\mathrm{~g})$ ). In the ciliary case, each flagellum has an asymmetric beat pattern [21]. In the power stroke, each flagellum extends and bends at the base, sweeping back like the arms of a person doing the breaststroke. On the recovery stroke, the flagellum folds, leading as we shall see below to less drag. When exposed to bright light, the alga swims in reverse, with its two flagella extended and propagating bending waves away from the cell body as in the case of sperm cells described above [57]. Paramecium is another classic example of a ciliated microorganism. Its surface is covered by thousands 
of cilia that beat in a coordinated manner [58], propelling the cell at speeds of $\approx 500 \mu \mathrm{m} \mathrm{s}^{-1}$ (figure 1(h)). Arrays of beating cilia are also found lining the airway where they sweep mucus and foreign particles up toward the nasal passage [59].

\section{Flows at low Reynolds number}

\subsection{General properties}

We first briefly discuss the general properties of flow at low Reynolds numbers. For more detail we refer to the classic monographs by Happel and Brenner [60], Kim and Karrila [61] and Leal [62]; an introduction is also offered by Hinch [63] and Pozrikidis [64].

To solve for the force distribution on an organism, we need to solve for the flow field $u$ and pressure $p$ in the surrounding fluid. For an incompressible Newtonian fluid with density $\rho$ and viscosity $\eta$, the flow satisfies the Navier-Stokes equations

$\rho\left(\frac{\partial}{\partial t}+\boldsymbol{u} \cdot \nabla\right) \boldsymbol{u}=-\nabla p+\eta \nabla^{2} \boldsymbol{u}, \quad \nabla \cdot \boldsymbol{u}=0$,

together with the boundary conditions appropriate to the problem at hand. In the case of swimming of a deformable body, the no-slip boundary condition states that the velocity of the fluid at the boundary is equal to the velocity of the material points on the body surface. The Navier-Stokes equations are a pointwise statement of momentum conservation. Once $\boldsymbol{u}$ and $p$ are known, the stress tensor is given by $\boldsymbol{\sigma}=-p \mathbf{1}+\eta[\nabla \boldsymbol{u}+$ $(\nabla \boldsymbol{u})^{\mathrm{T}}$ ] (1 is the identity tensor), and the hydrodynamic force $\boldsymbol{F}$ and torque $\boldsymbol{L}$ acting on the body are found by integrating over its surface $S$,

$\boldsymbol{F}(t)=\iint_{S} \boldsymbol{\sigma} \cdot \boldsymbol{n} \mathrm{d} S, \quad \boldsymbol{L}(t)=\iint_{S} \boldsymbol{x} \times(\boldsymbol{\sigma} \cdot \boldsymbol{n}) \mathrm{d} S$,

where $\boldsymbol{x}$ denotes positions on the surface $S$ and $\boldsymbol{n}$ the unit normal to $S$ into the fluid (in this paper torques are measured with respect to some arbitrary origin).

The Reynolds number is a dimensionless quantity which qualitatively captures the characteristics of the flow regime obtained by solving equation (1), and it has several different physical interpretations. Consider a steady flow with typical velocity $U$ around a body of size $L$. The Reynolds number $R e$ is classically defined as the ratio of the typical inertial terms in the Navier-Stokes equation, $\sim \rho \boldsymbol{u} \cdot \nabla \boldsymbol{u}$, to the viscous forces per unit volume, $\sim \eta \nabla^{2} u$. Thus, $R e=\rho L U / \eta$. A low Reynolds number flow is one for which viscous forces dominate in the fluid.

A second interpretation can be given as the ratio of time scales. The typical time scale for a local velocity perturbation to be transported convectively by the flow along the body is $t_{\mathrm{adv}} \sim L / U$, whereas the typical time scale for this perturbation to diffuse away from the body due to viscosity is $t_{\text {diff }} \sim \rho L^{2} / \eta$. We see therefore that $R e=t_{\text {diff }} / t_{\text {adv }}$, and a low Reynolds number flow is one for which fluid transport is dominated by viscous diffusion.

We can also interpret $R e$ as a ratio of forces on the body. A typical viscous stress on a bluff body is given by $\sigma_{\text {viscous }} \sim \eta U / L$, leading to a typical viscous force on the body of the form $f_{\text {viscous }} \sim \eta U L$. A typical inertial stress is given by a Bernoulli-like dynamic pressure, $\sigma_{\text {inertial }} \sim \rho U^{2}$, leading to an inertial force $f_{\text {inertial }} \sim \rho U^{2} L^{2}$. We see that the Reynolds number is given by $R e=f_{\text {inertial }} / f_{\text {viscous }}$, and therefore in a low Reynolds number flow the forces come primarily from viscous drag.

A fourth interpretation, more subtle, was offered by Purcell [14]. He noted that, for a given fluid, $\mathcal{F}=\eta^{2} / \rho$ has units of force, and that any body acted upon by the force $\mathcal{F}$ will experience a Reynolds number of unity, independent of its size. Indeed, it is easy to see that $R e=f_{\text {viscous }} / \mathcal{F}$ and $R e=\left(f_{\text {inertial }} / \mathcal{F}\right)^{1 / 2}$, and therefore a body with a Reynolds number of one will have $f_{\text {inertial }}=f_{\text {viscous }}=\mathcal{F}$. A body moving at low Reynolds number therefore experiences forces smaller than $\mathcal{F}$, where $\mathcal{F} \approx 1 \mathrm{nN}$ for water.

What are the Reynolds numbers for swimming microorganisms [3]? In water $\left(\rho \approx 10^{3} \mathrm{~kg} \mathrm{~m}^{-3}, \eta \approx\right.$ $10^{-3} \mathrm{~Pa} \mathrm{~s}$ ), a swimming bacterium such as $E$. coli with $U \approx$ $10 \mu \mathrm{m} \mathrm{s}^{-1}$ and $L \approx 1-10 \mu \mathrm{m}$ has a Reynolds number $R e \approx$ $10^{-5}-10^{-4}$. A human spermatozoon with $U \approx 200 \mu \mathrm{m} \mathrm{s}^{-1}$ and $L \approx 50 \mu \mathrm{m}$ moves with $R e \approx 10^{-2}$. The larger ciliates, such as Paramecium, have $U \approx 1 \mathrm{~mm} \mathrm{~s}^{-1}$ and $L \approx 100 \mu \mathrm{m}$, and therefore $R e \approx 0.1$ [13]. At these low Reynolds numbers, it is appropriate to study the limit $R e=0$, for which the Navier-Stokes equations (1) simplify to the Stokes equations

$$
-\nabla p+\eta \nabla^{2} \boldsymbol{u}=0, \quad \nabla \cdot \boldsymbol{u}=0
$$

Since swimming flows are typically unsteady, we implicitly assume the typical frequency $\omega$ is small enough so that the 'frequency Reynolds number' $\rho L \omega^{2} / \eta$ is also small. Note that equation (3) is linear and independent of time, a fact with important consequences for locomotion, as we discuss below.

Before closing this subsection, we point out an important property of Stokes flows called the reciprocal theorem [60]. It is a principle of virtual work which takes a particularly nice form thanks to the linearity of equation (3). Consider a volume of fluid $V$, bounded by a surface $S$ with outward normal $\boldsymbol{n}$, in which two solutions to equation (3) exist, $\boldsymbol{u}_{1}$ and $\boldsymbol{u}_{2}$, satisfying the same boundary conditions at infinity. If the stress fields of the two flows are $\sigma_{1}$ and $\sigma_{2}$, then the reciprocal theorem states that the mixed virtual works are equal:

$$
\iint_{S} \boldsymbol{u}_{1} \cdot \boldsymbol{\sigma}_{2} \cdot \boldsymbol{n} \mathrm{d} S=\iint_{S} \boldsymbol{u}_{2} \cdot \boldsymbol{\sigma}_{1} \cdot \boldsymbol{n} \mathrm{d} S
$$

\subsection{Motion of solid bodies}

When a solid body submerged in a viscous fluid is subject to an external force smaller than $\mathcal{F}$, it will move with a low Reynolds number. What determines its trajectory? Since equation (3) is linear, the relation between kinetics and kinematics is linear. Specifically, if the solid body is subject to an external force $\boldsymbol{F}$, and an external torque $\boldsymbol{L}$, it will move with velocity $\boldsymbol{U}$ and rotation rate $\Omega$ satisfying

$$
\left(\begin{array}{l}
\boldsymbol{F} \\
\boldsymbol{L}
\end{array}\right)=\left(\begin{array}{ll}
\boldsymbol{A} & \boldsymbol{B} \\
\boldsymbol{B}^{\mathrm{T}} & \boldsymbol{C}
\end{array}\right) \cdot\left(\begin{array}{l}
\boldsymbol{U} \\
\boldsymbol{\Omega}
\end{array}\right),
$$


or the inverse relation

$$
\left(\begin{array}{l}
\boldsymbol{U} \\
\boldsymbol{\Omega}
\end{array}\right)=\left(\begin{array}{ll}
\boldsymbol{M} & \boldsymbol{N} \\
\boldsymbol{N}^{\mathrm{T}} & \boldsymbol{O}
\end{array}\right) \cdot\left(\begin{array}{l}
\boldsymbol{F} \\
\boldsymbol{L}
\end{array}\right) .
$$

The matrix in equation (5) is the 'resistance' matrix of the body and the matrix of equation (6) is the 'mobility' matrix. The reciprocal theorem (4) forces these matrices to be symmetric [60]. Dimensionally, since low-Re stresses scale as $\sim \eta U / L$, the sub-matrices scale as $[A] \sim \eta L$, $[B] \sim \eta L^{2},[C] \sim \eta L^{3}$, and similarly $[M] \sim(\eta L)^{-1}$, $[\boldsymbol{N}] \sim\left(\eta L^{2}\right)^{-1},[\boldsymbol{O}] \sim\left(\eta L^{3}\right)^{-1}$. For example, a solid sphere of radius $R$ has isotropic translational and rotational drag: $M=A^{-1}=(6 \pi \eta R)^{-1} \mathbf{1}$ and $\boldsymbol{O}=C^{-1}=\left(8 \pi \eta R^{3}\right)^{-1} \mathbf{1}$; the cross-couplings $\boldsymbol{N}$ and $\boldsymbol{B}$ vanish by symmetry. But for most problems, the details of the geometry of the body make these matrices very difficult to calculate analytically.

Three important features of equations (5)-(6) should be emphasized for their implications for locomotion. The first important property is drag anisotropy: the matrices $\boldsymbol{A}, \boldsymbol{M}$, $\boldsymbol{C}$ and $\boldsymbol{O}$ need not be isotropic (proportional to $\mathbf{1}$ ). As we discuss in section 4, drag anisotropy is a crucial ingredient without which biological locomotion could not occur at zero Reynolds number. For a simple illustration, consider a slender prolate spheroid of major axis $L$ and minor axis $\ell$ with $L \gg \ell$. If $e$ denotes the direction along the major axis of the spheroid, we have $A=A_{\|} e e+A_{\perp}(1-e e)$, with $A_{\|} \approx 4 \pi \eta L /[\ln (2 L / \ell)-1 / 2]$ and $A_{\perp} \approx 8 \pi \eta L[\ln (2 L / \ell)+$ $1 / 2]$. An interesting consequence of the drag anisotropy is the sedimentation of a spheroid under the action of gravity. If the body is not exactly parallel or perpendicular to the direction of gravity, then its sedimentation velocity makes a constant angle with the direction of gravity, and the spheroid slides along its length as it sediments.

Secondly, there exist geometries for which the matrices $\boldsymbol{B}$ and $\boldsymbol{N}$ are non-zero, namely chiral bodies, which lack a mirror symmetry plane. In that case, there is the possibility of driving translational motion through angular forcing-this strategy is employed by bacteria with rotating helical flagella (see section 6).

Thirdly, these matrices are important for calculating the diffusion constants of solid bodies. The fluctuation-dissipation theorem states that, in thermal equilibrium at temperature $T$, the translational diffusion constant of a solid body is given by the Stokes-Einstein relationship $D=k_{\mathrm{B}} T M$, where $k_{\mathrm{B}}$ is Boltzmann's constant, while the rotational diffusion constant is given by $D_{\mathrm{R}}=k_{\mathrm{B}} T \boldsymbol{O}$. The typical time scale for a body to move by diffusion along its length is $\tau_{\mathrm{D}} \sim L^{2} /[D]$, while $\tau_{\mathrm{R}} \sim\left[\boldsymbol{D}_{\mathrm{R}}\right]^{-1}$ is the typical time scale for the reorientation of the cell by thermal forces alone. For a non-motile $E$. coli bacterium at room temperature, we have $[D] \sim 0.1 \mu \mathrm{m}^{2} \mathrm{~s}^{-1}$ in water; while the time scale for thermal reorientation of the cell axis, $\left[\boldsymbol{D}_{\mathrm{R}}\right]^{-1}$, is a few minutes.

\subsection{Flow singularities}

Since the Stokes' equations, equation (3), are linear, traditional mathematical methods relying on linear superposition may be used to solve for flow and pressure fields. Green's function for
Stokes flow with a Dirac-delta forcing of the form $\delta\left(\boldsymbol{x}-\boldsymbol{x}^{\prime}\right) \boldsymbol{F}$ is given by

$$
\begin{gathered}
\boldsymbol{u}(\boldsymbol{x})=\boldsymbol{G}\left(\boldsymbol{x}-\boldsymbol{x}^{\prime}\right) \cdot \boldsymbol{F}, \\
\text { with } \boldsymbol{G}(\boldsymbol{r})=\frac{1}{8 \pi \eta}\left(\frac{1}{r}+\frac{\boldsymbol{r} \boldsymbol{r}}{r^{3}}\right), \quad r=|\boldsymbol{r}|, \\
p(\boldsymbol{x})=\boldsymbol{H}\left(\boldsymbol{x}-\boldsymbol{x}^{\prime}\right) \cdot \boldsymbol{F}, \quad \text { with } \boldsymbol{H}(\boldsymbol{r})=\frac{\boldsymbol{r}}{4 \pi r^{3}} .
\end{gathered}
$$

The tensor $G$ is known as the Oseen tensor, and the fundamental solution, equation (7), is termed a stokeslet [31]. Physically, it represents the flow field due to a point force, $\boldsymbol{F}$, acting on the fluid at the position $\boldsymbol{x}^{\prime}$ as a singularity. The velocity field is seen to decay in space as $1 / r$, a result which can also be obtained by dimensional analysis. Indeed, for a three-dimensional force $\boldsymbol{F}$ acting on the fluid, and by linearity of Stokes' flow, the flow velocity has to take the form $u \sim F f(\theta, r, \eta)$, where $\theta$ is the angle between the direction of $\boldsymbol{F}$ and $\boldsymbol{r}$, and where $F$ is the magnitude of the force. Dimensional analysis leads to $u \sim g(\theta) F / \eta r$ with a $1 / r$ decay.

An important property of the stokeslet solution for locomotion is directional anisotropy. Indeed, we see from equation (7) that if we evaluate the velocity in the direction parallel to the applied force, we obtain that $u_{\|}=F / 4 \pi \eta r$, whereas the velocity in the direction perpendicular to the force is given by $u_{\perp}=F / 8 \pi \eta r$. For the same applied force, the flow field in the parallel direction is therefore twice that in the perpendicular direction $\left(u_{\|}=2 u_{\perp}\right)$. Alternatively, to obtain the same velocity, one would need to apply a force in the perpendicular direction twice as large as in the parallel direction $\left(F_{\perp}=2 F_{\|}\right)$. Such anisotropy, which is reminiscent of the anisotropy in the mobility matrix for long slender bodies (section 3.2; see also section 5.2) is at the origin of the drag-based propulsion method employed by swimming microorganisms (see section 4.3).

From the fundamental solution above, equation (7), the complete set of singularities for viscous flow can be obtained by differentiation [65]. One derivative leads to force dipoles, with flow fields decaying as $1 / r^{2}$. Two derivatives lead to source dipole (potential flow also known as a doublet), and force quadrupoles, with velocity decaying in space as $1 / r^{3}$. Higher-order singularities are easily obtained by subsequent differentiation.

A well-chosen distribution of such singularities can then be used to solve exactly Stokes' equation in a variety of geometries. For example, the Stokes flow past a sphere is a combination of a stokeslet and a source dipole at the center of the sphere [66]. For spheroids, the method was pioneered by Chwang and $\mathrm{Wu}[65]$, and we refer to [61, 67] for a textbook treatment. A linear superposition of singularities is also at the basis of the boundary integral method to computationally solve for Stokes flows using solely velocity and stress information at the boundary (see $[64,67]$ ).

When a flow field is described by a number of different flow singularities, the singularity with the slowest spatial decay is the one that dominates in the far field. Since a cell swimming in a viscous fluid at low Reynolds numbers is force- and torquefree (equation (9)), the flow singularities that describe pointforces (stokeslets) and point-torques (antisymmetric force 
dipole or rotlets) cannot be included in the far-field description. As a result, the flow field far from a swimming cell is in general well represented by a symmetric force dipole or stresslet [68]. Such far-field behavior has important consequences on cellcell hydrodynamic interactions as detailed in section 7.1.

Finally, flow singularities are modified by the presence of solid boundaries. Consider for example a flow near a planar wall. In general the no-slip boundary condition along the wall is not satisfied by the original set of singularities. As a result, and similarly to the method of images in electrostatics, the flow in the presence of the wall is then the linear superposition of the infinite-fluid flow, plus a sequence of image flow fields located on the other side of the surface, whose types and strengths are such that the correct boundary condition is enforced along the wall. Image flow fields for the fundamental singularities of Stokes flows were pioneered by Blake $[69,70]$, and a textbook treatment is offered in $[61,64]$. In contrast to the method of images in electrostatics, a mathematical complication stems from the fact that in fluid mechanics the no-slip boundary condition is vectorial, whereas the constant-potential boundary condition in electrostatics is scalar. For a stokeslet near wall, the image flow field is a stokeslet, a force dipole and a source dipole $[69,70]$. These images can be used to show that the mobility of a sphere in translation decreases near a wall, and that the mobility for translation along the wall is larger than the mobility for translation perpendicular to the wall $[60,61]$.

\section{Life at low Reynolds number}

We now consider the general problem of self-propelled motion at low Reynolds number. We call a body a 'swimmer' if by deforming its surface it is able to sustain movement through fluid in the absence of external (non-hydrodynamic) forces and torques. Note that the 'body' includes appendages such as the cilia covering a Paramecium or the helical flagella of E. coli.

\subsection{Reinterpreting the Reynolds number}

We first offer an alternative interpretation of the Reynolds number in the context of swimming motion. Let us consider a swimmer of mass $m$ and size $L$ swimming with velocity $U$ through a viscous fluid of density $\rho$ and viscosity $\eta$. Suppose the swimmer suddenly stops deforming its body, it will then decelerate according to Newton's law $m a=f_{\text {drag }}$. What is the typical length scale $d$ over which the swimmer will coast due to the inertia of its movement? For motion at high Reynolds number, as in the case of a human doing the breaststroke, the typical drag is $f_{\text {drag }}=f_{\text {inertial }} \sim \rho U^{2} L^{2}$, leading to a deceleration $a \sim \rho U^{2} L^{2} / m$. The swimmer coasts a length $d \sim U^{2} / a \sim m /\left(\rho L^{2}\right)$. If the swimmer has a density $\rho_{\mathrm{s}} \sim m / L^{3}$, we see that the dimensionless coasting distance is given by the ratio of densities, $d / L \sim \rho_{\mathrm{s}} / \rho$. A human swimmer in water can coast for a couple of meters. In contrast, for motion at low Reynolds number, the drag force has the viscous scaling, $f_{\text {drag }}=f_{\text {viscous }} \sim \eta U L$, and the swimmer can coast a distance $d / L \sim \operatorname{Re} \rho_{\mathrm{s}} / \rho$. For a swimming bacterium such as E. coli, this argument leads to $d \approx 0.1 \mathrm{~nm}$ [14]. For $R e<1$, the Reynolds number can therefore be interpreted as a non-dimensional coasting distance.
A consequence of this analysis is that in a world of low Reynolds number, the response of the fluid to the motion of boundaries is instantaneous. This conclusion was anticipated by our second interpretation of the Reynolds number (section 3), where we saw that in the limit of very low $R e$, velocity perturbations diffuse rapidly relative to the rate at which fluid particles are carried along by the flow. To summarize, the rate at which the momentum of a low-Re swimmer is changing is completely negligible when compared with the typical magnitude of the forces from the surrounding viscous fluid. As a result, Newton's law becomes a simple statement of instantaneous balance between external and fluid forces and torques

$$
\boldsymbol{F}_{\mathrm{ext}}(t)+\boldsymbol{F}(t)=\mathbf{0}, \quad \boldsymbol{L}_{\mathrm{ext}}(t)+\boldsymbol{L}(t)=\mathbf{0} .
$$

In most cases, there are no external forces, and $\boldsymbol{F}_{\text {ext }}(t)=\mathbf{0}$. Situations where $\boldsymbol{L}_{\text {ext }}(t)$ is non-zero include the locomotion of nose-heavy or bottom-heavy cells [71]; in all other cases we will assume $\boldsymbol{L}_{\text {ext }}(t)=\mathbf{0}$.

\subsection{The swimming problem}

Mathematically, the swimming problem is stated as follows. Consider a body submerged in a viscous fluid. In a reference frame fixed with respect to some arbitrary reference point in its body, the swimmer deforms its surface in a prescribed time-varying fashion given by a velocity field on its surface, $\boldsymbol{u}_{S}(t)$. The velocity field $\boldsymbol{u}_{S}(t)$ is the 'swimming gait'. A swimmer is a deformable body by definition, but it may be viewed at every instant as a solid body with unknown velocity $\boldsymbol{U}(t)$ and rotation rate $\boldsymbol{\Omega}(t)$. The instantaneous velocity on the swimmer's surface is therefore given by $\boldsymbol{u}=\boldsymbol{U}+\boldsymbol{\Omega} \times \boldsymbol{x}+\boldsymbol{u}_{S}$, which, due to the no-slip boundary condition, provides the boundary conditions needed to solve equation (3). The unknown values of $\boldsymbol{U}(t)$ and $\boldsymbol{\Omega}(t)$ are determined by satisfying equation (9).

The mathematical difficulty of the swimming problem arises from having to solve for the Stokes flow with unknown boundary condition; in that regard, low-Re swimming is reminiscent of an eigenvalue problem. A great simplification was derived by Stone and Samuel [72], who applied the reciprocal theorem, equation (4), to the swimming problem. Recall that the reciprocal theorem involves two different flow problems for the same body. Let $\boldsymbol{u}$ and $\boldsymbol{\sigma}$ be the velocity and stress fields we seek in the swimming problem. For the second flow problem, suppose $\hat{\boldsymbol{u}}$ and $\hat{\boldsymbol{\sigma}}$ are the velocity and stress fields for the dual problem of instantaneous solid body motion of the swimmer with velocity $\hat{\boldsymbol{U}}$ and rotation rate $\hat{\boldsymbol{\Omega}}$. This problem correspond to subjecting the shape, $S(t)$, to an external force, $\hat{\boldsymbol{F}}$, and torque, $\hat{\boldsymbol{L}}$, instantaneously. Applying the reciprocal theorem, equation (4), we obtain the integral relationship [72]

$$
\hat{\boldsymbol{F}} \cdot \boldsymbol{U}+\hat{\boldsymbol{L}} \cdot \boldsymbol{\Omega}=-\iint_{S(t)} \boldsymbol{u}_{S} \cdot \hat{\boldsymbol{\sigma}} \cdot \boldsymbol{n} \mathrm{d} S .
$$

Equation (10) shows explicitly how the swimming velocity $\boldsymbol{U}$ and rotation rate $\Omega$ may be found instantaneously in terms of 
the gait $\boldsymbol{u}_{S}$, given the solution to the dual problem of the flow induced by the motion of the rigid body with instantaneous shape $S(t)$, subject to force $\hat{\boldsymbol{F}}$ and torque $\hat{\boldsymbol{L}}$. Since $\hat{\boldsymbol{F}}$ and $\hat{\boldsymbol{L}}$ are arbitrary, equation (10) provides enough equations to solve for all components of the swimming kinematics. Note that in equation (10), all four fields $\hat{\boldsymbol{F}}, \boldsymbol{U}, \hat{\boldsymbol{L}}$ and $\boldsymbol{\Omega}$ are implicitly time dependent through the time-dependence of the shape $S(t)$. Note also that for squirming motion, where the shape of the swimmer surface remains constant and all deformation is tangential to the surface $\left(\boldsymbol{u}_{S} \cdot \boldsymbol{n}=0\right)$, equation (10) simplifies further. For a spherical squirmer of radius $R$, we have the explicit formulae [72]

$$
\begin{gathered}
\boldsymbol{U}=-\frac{1}{4 \pi R^{2}} \iint_{S} \boldsymbol{u}_{S} \mathrm{~d} S, \\
\boldsymbol{\Omega}=-\frac{3}{8 \pi R^{3}} \iint_{S} \boldsymbol{n} \times \boldsymbol{u}_{S} \mathrm{~d} S .
\end{gathered}
$$

\subsection{Drag-based thrust}

Most biological swimmers exploit the motion of slender appendages such as flagella or cilia for locomotion. This limit of slender bodies allows us to provide a physical, intuitive way to understand the origin of locomotion through drag, which we now present; the specifics of biological and artificial flagellar actuation will be discussed in section 6 .

The fundamental property allowing for drag-based thrust of slender filaments is their drag anisotropy, as introduced in section 3.2. Indeed, consider a thin filament immersed in a viscous fluid which is motionless but for flows induced by the motion and deformation of the filament. The shape of the filament is described by its tangent vector $t(s)$ at distance $s$ along the filament, and its instantaneous deformation is described by the velocity field $\boldsymbol{u}(s, t)$, where $t$ is time. For asymptotically slender filaments, (see section 5), as in the case of prolate spheroids, the local viscous drag force per unit length opposing the motion of the filament is

$$
f=-\xi_{\|} u_{\|}-\xi_{\perp} u_{\perp}
$$

where $\boldsymbol{u}_{\|}$and $\boldsymbol{u}_{\perp}$ are the projections of the local velocity on the directions parallel and perpendicular to the filament, i.e. $\boldsymbol{u}_{\|}=(\boldsymbol{u} \cdot \boldsymbol{t}) \boldsymbol{t}$ and $\boldsymbol{u}_{\perp}=\boldsymbol{u}-\boldsymbol{u}_{\|} ; \xi_{\|}$and $\xi_{\perp}$ are the corresponding drag coefficients (typically $\xi_{\perp} / \xi_{\|} \approx 2$ ).

The origin of drag-based thrust relies on the following two physical ideas: (a) the existence of drag anisotropy means that propulsive forces can be created at a right angle with respect to the local direction of motion of the filament and (b) a filament can deform in a time-periodic way and yet create non-zero time-averaged propulsion. To illustrate these ideas, consider the beating filament depicted in figure 2. Any short segment of the filament may be regarded as straight and moving with velocity $\boldsymbol{u}$ at an angle $\theta$ with the local tangent (figure 2, inset). This velocity resolves into components $u_{\|}=u \cos \theta$ and $u_{\perp}=u \sin \theta$, leading to drag per unit length components $f_{\|}=-\xi_{\|} u_{\|}=-\xi_{\|} u \cos \theta$ and $f_{\perp}=-\xi_{\perp} u_{\perp}=-\xi_{\perp} u \sin \theta$. For isotropic drag, $\xi_{\|}=\xi_{\perp}$, and the force on the filament has the same direction as the velocity of the filament; however, if

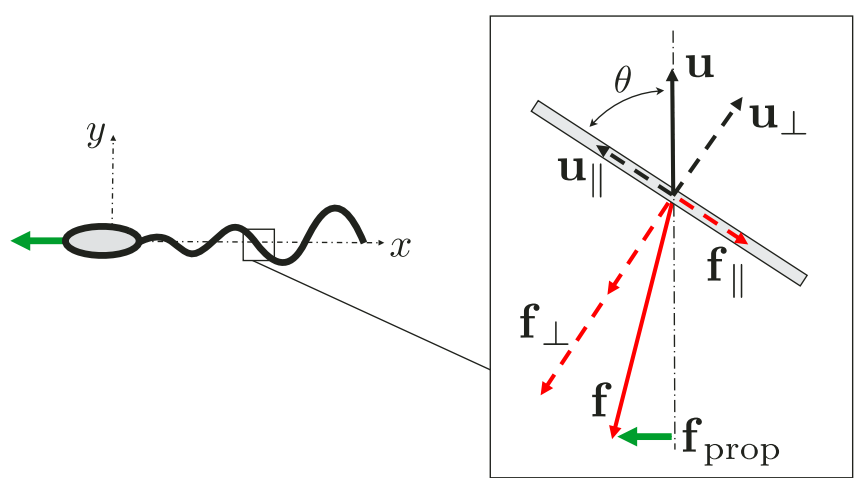

Figure 2. Physics of drag-based thrust: the drag anisotropy for slender filaments provides a means to generate forces perpendicular to the direction of the local actuation (see text for notation)

$\xi_{\|} \neq \xi_{\perp}$, the drag per unit length on the filament includes a component $f_{\text {prop }}$ which is perpendicular to the direction of the velocity,

$$
\boldsymbol{f}_{\text {prop }}=\left(\xi_{\|}-\xi_{\perp}\right) u \sin \theta \cos \theta \boldsymbol{e}_{x} .
$$

In addition, in order to generate a net propulsion from a time-periodic movement, we see from equation (13) that both the filament velocity $u$ and its orientation angle $\theta$ need to vary periodically in time. For example, actuation with a given $\boldsymbol{u}$ and $\theta$, followed by the change $\boldsymbol{u} \rightarrow-\boldsymbol{u}$ and $\theta \rightarrow \pi-\theta$, leads to a propulsive force with a constant sign; in contrast, actuation in which only $\boldsymbol{u} \rightarrow-\boldsymbol{u}$ changes periodically leads to zero average force.

It is important to realize that two properties are crucial for propulsion. The first property is purely local, and states that with the appropriate geometry and actuation, a force can be created in the direction perpendicular to the motion of the filament. This conclusion relies explicitly on the properties of Stokes flows, and in a world with isotropic viscous friction $\left(\xi_{\perp}=\xi_{\|}\right)$, locomotion would not be possible [32,73]. The second property is a global condition that says that the periodic actuation of the filament needs to be non-time reversible in order to generate non-zero forces on average; this property is generally known as Purcell's scallop theorem [14], which we now describe.

\subsection{The scallop theorem}

As pointed out above, the Stokes equation-equation (3) is linear and independent of time. These properties lead to kinematic reversibility, an important and well-known symmetry property associated with the motion of any body at zero Reynolds number $[60,62]$. Consider the motion of a solid body with an instantaneous prescribed velocity $\boldsymbol{U}$ and rotation rate $\Omega$ together with the flow field surrounding it. If we apply the scaling $\boldsymbol{U} \rightarrow \alpha \boldsymbol{U}$ and $\boldsymbol{\Omega} \rightarrow \alpha \boldsymbol{\Omega}$, then by linearity, the entire flow and pressure field transform as $\boldsymbol{u} \rightarrow \alpha \boldsymbol{u}$ and $p \rightarrow \alpha p$. Consequently, the instantaneous flow streamlines remain identical, and the fluid stresses undergo a simple linear scaling, resulting in the symmetry $\boldsymbol{F} \rightarrow \alpha \boldsymbol{F}$ and $\boldsymbol{L} \rightarrow \alpha \boldsymbol{L}$ for the force and torque acting on the body. In particular, when $\alpha=-1$, this means that an instantaneous reversing of 
the forcing does not modify the flow patterns, but only the direction in which they are occurring.

When applied to low Reynolds number locomotion, the linearity and time-independence of Stokes equation of motion lead to two important properties [14]. The first is rate independence: if a body undergoes surface deformation, the distance travelled by the swimmer between two different surface configurations does not depend on the rate at which the surface deformation occurs but only on its geometry (i.e. the sequence of shapes the swimmer passes through between these two configurations).

A mathematical proof of this statement can be outlined as follows. We consider for simplicity swimmers with no rotational motion (an extension to the case $\Omega \neq \mathbf{0}$ is straightforward). Consider a body deforming its surface between two different configurations identified by times $t_{0}$ and $t_{1}$. We denote by $\boldsymbol{r}_{S}$ the positions of points on the surface of the swimmer. From equation (10), we know that the instantaneous speed of locomotion is given by a general integral of the form

$$
\boldsymbol{U}(t)=\iint_{f\left(r_{S}\right)} \dot{\boldsymbol{r}}_{S} \cdot \boldsymbol{g}\left(\boldsymbol{r}_{S}\right) \mathrm{d} S,
$$

where the function $\boldsymbol{g}\left(\boldsymbol{r}_{S}\right)$ is a general tensor depending on the shape of the body and on the boundary conditions at infinity (but in general not known analytically) and where we have used $\boldsymbol{u}_{S}=\dot{\boldsymbol{r}}_{S}=\mathrm{d} \boldsymbol{r}_{S} / \mathrm{d} t$. The net motion of the swimmer between $t_{0}$ and $t_{1}$ is therefore given by

$$
\Delta \boldsymbol{X}=\int_{t_{0}}^{t_{1}} \boldsymbol{U}(t) \mathrm{d} t .
$$

Now consider the same succession of swimmer shapes, but occurring at a different rate. This new history of shapes $\boldsymbol{r}_{S}^{\prime}\left(t^{\prime}\right)$ is related to the old by a mapping $t^{\prime}=f(t)$, with $t_{i}=f\left(t_{i}\right)$ for $i=0,1$ and $r_{S}^{\prime}\left(t^{\prime}\right)=r_{S}(t)$ for all times. We now have

$$
\Delta \boldsymbol{X}^{\prime}=\int_{t_{0}}^{t_{1}} \boldsymbol{U}^{\prime}\left(t^{\prime}\right) \mathrm{d} t^{\prime}=\int_{t_{0}}^{t_{1}} \boldsymbol{U}^{\prime}(f(t)) f^{\prime}(t) \mathrm{d} t,
$$

where the chain rule implies

$$
\begin{gathered}
\boldsymbol{U}^{\prime}\left(t^{\prime}\right) f^{\prime}(t)=\iint_{f\left(\boldsymbol{r}_{S}^{\prime}\right)} \frac{\mathrm{d} \boldsymbol{r}_{S}^{\prime}}{\mathrm{d} t^{\prime}} f^{\prime}(t) \cdot \boldsymbol{g}\left(\boldsymbol{r}_{S}\right) \mathrm{d} S \\
=\iint_{f\left(\boldsymbol{r}_{S}\right)} \frac{\mathrm{d} \boldsymbol{r}_{S}}{\mathrm{~d} t} \cdot \boldsymbol{g}\left(\boldsymbol{r}_{S}\right) \mathrm{d} S .
\end{gathered}
$$

We see therefore that $\boldsymbol{U}^{\prime}\left(t^{\prime}\right) f^{\prime}(t)=\boldsymbol{U}(t)$, and therefore $\Delta \boldsymbol{X}=\Delta \boldsymbol{X}^{\prime}$. The net distance traveled by the swimmer does not depend on the rate at which it is being deformed, but only on the geometrical sequence of shape. One consequence of this property is that many aspects of low Reynolds number locomotion can be addressed using a purely geometrical point of view [74-78].

The second important property of swimming without inertia is the so-called scallop theorem: if the sequence of shapes displayed by a swimmer deforming in a timeperiodic fashion is identical when viewed after a time-reversal transformation, then the swimmer cannot move on average. Note that the condition is not that the motion be strictly time-reversal invariant, with the same forward and backwards rate, but only that the sequence of shapes is the same when viewed forward or backward in time. This class of surface deformations is termed 'reciprocal deformation'. The scallop theorem puts a strong geometrical constraint on the type of swimming motion which is effective at low Reynolds numbers.

An outline of the proof follows. Again, we consider purely translational motion for simplicity. Let us consider a swimmer deforming its body in a reciprocal manner between times $t_{0}$ and $t_{1}$, with a sequence of shapes $\boldsymbol{r}_{S}(t)$. Assume that $\boldsymbol{r}_{S}\left(t_{0}\right)=\boldsymbol{r}_{S}\left(t_{1}\right)$ so that the period of the surface deformation is $t_{1}-t_{0}$. Let $t_{2}$ be the instant of time between $t_{0}$ and $t_{1}$ that divides the sequence of shapes into the forward and backward sequence. The sequence of shapes at time $t^{\prime}$ between $t_{2}$ and $t_{1}$ is given by the map $t^{\prime}=f(t)$ for $t$ between $t_{0}$ and $t_{2}$, with $t_{2}=f\left(t_{2}\right)$ and $t_{1}=f\left(t_{0}\right): r_{S}^{\prime}\left(t^{\prime}\right)=r_{S}(t)$. The net distance traveled by the swimmer over any interval is given by equations similar to equations (14) and (15). Using the chain rule again, we find that

$$
\begin{gathered}
\Delta \boldsymbol{X}_{t_{2} \rightarrow t_{1}}^{\prime}=\int_{t_{2}}^{t_{1}} \boldsymbol{U}^{\prime}\left(t^{\prime}\right) \mathrm{d} t^{\prime}=\int_{t_{2}}^{t_{0}} \boldsymbol{U}(t) \mathrm{d} t \\
=-\int_{t_{0}}^{t_{2}} \boldsymbol{U}(t) \mathrm{d} t=-\Delta \boldsymbol{X}_{t_{0} \rightarrow t_{2}},
\end{gathered}
$$

and the net distance traveled in a period vanishes, $\Delta \boldsymbol{X}_{t_{0} \rightarrow t_{2}}+$ $\Delta \boldsymbol{X}_{t_{2} \rightarrow t_{1}}^{\prime}=\mathbf{0}$. Reciprocal motion cannot be used for locomotion at low Reynolds numbers. Note that we did not need to assume anything about the geometry of the fluid surrounding the swimmer; the scallop theorem remains valid near solid walls, and more generally in confined environments. However, the scallop theorem does not hold for a body making reciprocal motions near a flexible object, such as a wall or another swimmer, since in that case equation (10) must be modified to include $\boldsymbol{u}_{S}$ at the surface of the flexible object $[79,80]$.

In his original article, Purcell illustrated the futility of reciprocal motion with the example of a scallop, a mollusk that opens and closes its shell periodically. Independent of the rate of opening and closing, a low-Reynolds number scallop cannot swim ${ }^{3}$. Another example of a reciprocal deformation is a dumbbell, made of two solid spheres separated by timeperiodic distance. More generally, bodies with a single degree of freedom deform in a reciprocal fashion, and cannot move on average.

Successful low-Re swimmers therefore must display non-reciprocal body kinematics. In his original paper, Purcell proposed a simple example of non-reciprocal body deformation, a two-hinged body composed of three rigid links rotating out of phase with each other, now referred to as Purcell's swimmer [73]. Another elementary example is a trimer, made of three rigid spheres whose separation distances vary in a time-periodic fashion with phase differences [82]. More examples are discussed in section 9. Note that, mathematically, the presence of non-reciprocal kinematics is a necessary but not sufficient condition to obtain propulsion. A simple counterexample is a configuration with two swimmers

3 A real scallop actually swims at high Reynolds number, a regime for which the constraints of the theorem of course do not apply [81]. 
which are mirror-images of each other and arranged head-tohead; although the kinematics of the two bodies taken together is non-reciprocal, the mirror symmetry forbids net motion of their center of mass.

For biological bodies deforming in a continuous fashion, the prototypical non-reciprocal deformation is a wave. Consider a continuous filament of length $L$ deforming with small amplitude $y(x, t)$ (i.e. for which $|\partial y / \partial x| \ll 1)$; in that case, the propulsive force generated along the filament, equation (13), is given by

$$
\boldsymbol{F}_{\text {prop }} \approx\left(\xi_{\perp}-\xi_{\|}\right) \int_{0}^{L}\left(\frac{\partial y}{\partial t} \frac{\partial y}{\partial x}\right) \mathrm{d} x \boldsymbol{e}_{x} .
$$

If the filament deforms as a planar wave traveling in the $x$-direction, $y(x, t)=f(x-c t)$, the force is given by $\boldsymbol{F}_{\text {prop }}=c\left(\xi_{\|}-\xi_{\perp}\right) \int f^{2}(\eta) \mathrm{d} \eta \boldsymbol{e}_{x}$ and propulsion is seen to occur in the direction opposite to that of the wave: $c F_{\text {prop }}<0$. Mathematically, a wave-like deformation allows the product $(\partial y / \partial t \cdot \partial y / \partial x)$ to keep a constant sign along the entire filament, and therefore all portions of the filament contribute to generating propulsion. In general, all kinds of three-dimensional wave-like deformations lead to propulsion, including helical waves of flexible filaments [83].

Finally, it is worth emphasizing that the scallop theorem is strictly valid in the limit where all the relevant Reynolds numbers in the swimming problem are set to zero. Much recent work has been devoted to the breakdown of the theorem with inertia, and the transition from the Stokesian realm to the Eulerian realm is found to be either continuous or discontinuous depending on the spatial symmetries in the problem considered [84-91].

\section{Historical studies, and further developments}

In this section we turn to the first calculations of the swimming velocities of model microorganisms. We consider two simple limits: (1) propulsion by small-amplitude deformations of the surface of the swimmer and (2) propulsion by the motion of a slender filament. Although these limits are highly idealized, our calculations capture essential physical aspects of swimming that are present in more realistic situations.

\subsection{Taylor's swimming sheet}

In 1951, Taylor asked how a microorganism could propel itself using viscous forces, rather than imparting momentum to the surrounding fluid as fish do [30]. To answer this question, he calculated the flow induced by propagating transverse waves of small amplitude on a sheet immersed in a viscous fluid. In this subsection, we review Taylor's calculation [30]. The sheet is analogous to the beating flagellum of spermatazoa, but since the flow is two-dimensional, the problem of calculating the induced flow is greatly simplified. The height of the sheet over the plane $y=0$ is

$$
h=b \sin (k x-\omega t),
$$

where the $x$-direction is parallel to the direction of propagation of the wave, $b$ is the amplitude, $k$ is the wavenumber and $\omega$ is the frequency of the oscillation. Note that we work in the reference frame in which the material points of the sheet move up and down, with no $x$-component of motion. The problem is further simplified by the assumption that the amplitude is small compared with the wavelength $2 \pi / k$. Note that the motion descried by equation (20) implies that the sheet is extensible. If the sheet is inextensible, then the material points of the sheet make narrow figure eights instead of moving up and down; nevertheless the extensible and inextensible sheets have the same swimming velocity to leading order in $b k$.

To find the flow induced by the traveling-wave deformation, we need to solve the Stokes equations with noslip boundary conditions at the sheet,

$$
\boldsymbol{u}(x, h(x, t))=\frac{\partial h}{\partial t} \boldsymbol{e}_{y}=-b \omega \cos (k x-\omega t) \boldsymbol{e}_{y},
$$

with an unknown but uniform and steady flow far from the sheet,

$$
\lim _{y \rightarrow \infty} \boldsymbol{u}(x, y)=-\boldsymbol{U} .
$$

Since we work in the rest frame of the sheet, $\boldsymbol{U}$ is the swimming velocity of the sheet in the laboratory frame, in which the fluid is at rest at $y \rightarrow \infty$. Although it turns out in this problem that the leading-order swimming speed is steady in time, other situations lead to unsteady swimming speeds. In all cases we are free to use non-inertial frames-even rotating frameswithout introducing fictitious forces, since inertia may be disregarded at zero Reynolds number.

Although $\boldsymbol{U}$ is unknown, Taylor found that no additional conditions are required to determine $U$; instead, there is a unique value of $\boldsymbol{U}$ consistent with the solution to the Stokes equations and the no-slip boundary condition (21). It is also important to note that although the Stokes equations are linear, the swimming speed $\boldsymbol{U}$ is not a linear function of the amplitude $b$, since $b$ enters the no-slip boundary condition both on the right-hand side of equation (21), and implicitly on the left-hand side of equation (21) through equation (20). In fact, symmetry implies that the swimming speed $\boldsymbol{U}$ must be an even function of $b$. Replacing $b$ by $-b$ amounts to translating the wave (20) by half a wavelength. But any translation of the wave cannot change the swimming speed; therefore, $\boldsymbol{U}$ is even in $b$.

Taylor solved the swimming problem by expanding the boundary condition (21) in $b k$, and solving the Stokes equations order by order. We will consider the leading term only, which, as argued above, is quadratic in $b$. Since the swimming velocity $\boldsymbol{U}$ is a vector, it must be proportional to the only other vector in the problem, the wavevector $\boldsymbol{k}=k \boldsymbol{e}_{x}$. For example, if we were to consider the superposition of two traveling waves on the sheet, propagating in different directions, we would expect the swimming direction to be along the vector sum of the corresponding wavevectors. Dimensional analysis determines the remaining dependence of $\boldsymbol{U}$ on the parameters of the problem: $\boldsymbol{U} \propto \omega \boldsymbol{k} b^{2}$. Taylor's calculation yields the proportionality constant, with sign

$$
\boldsymbol{U}=-\frac{1}{2} \omega \boldsymbol{k} b^{2} .
$$

Note that dimensional considerations require the swimming speed to be independent of viscosity. This result holds 


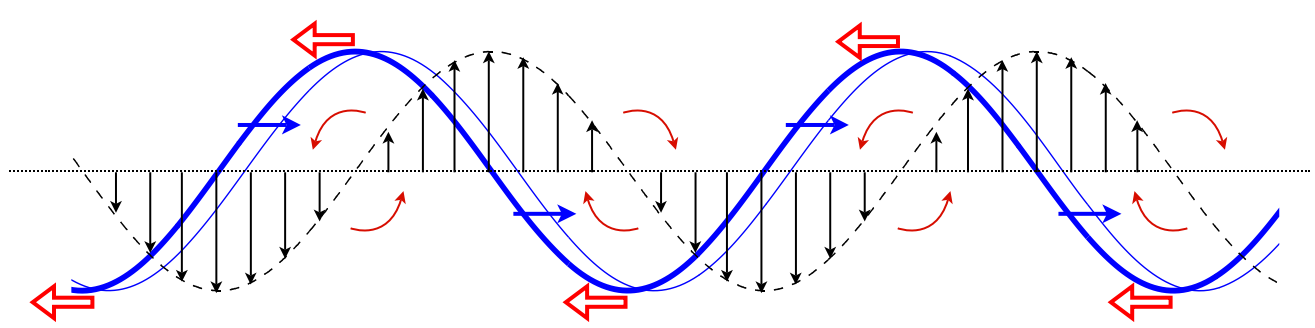

Figure 3. Physical interpretation of the swimming direction for Taylor's swimming sheet. As the wave of deformation is traveling to the right (blue solid lines and rightward arrows), it leads to vertical displacements of the material points on the sheet (black vertical arrows and dashed line) which induce flow vorticity of alternating signs (curved red arrows), and a leftward motion of the sheet (red leftward arrows).

due to our assumption that the waveform (20) is prescribed, independent of the load. However, the rate $W$ that the sheet does work on the fluid does depend on viscosity. The net force per wavelength exerted by the sheet on the fluid vanishes, but by integrating the local force per area $(\sim \eta \omega b k)$ against the local velocity $(\sim \omega b)$, Taylor found

$$
W=\eta \omega^{2} k b^{2} .
$$

Note that only the first-order solution for the flow is required to calculate $W$. In section 6 we consider more realistic models that account for the internal mechanisms that generate the deformation of the swimmer. Such models can predict a viscosity dependence in the swimming speed, since the shape of the beating filament may depend on viscosity [92-94]. And in section 8 we show how the speed of a swimmer in a complex fluid can depend on material parameters, even for the swimming problem with prescribed waveform.

According to equation (23), the swimmer moves in the direction opposite to the traveling wave. It is instructive to also consider the case of a longitudinal wave, in which the material points in the frame of the sheet undergo displacement $\delta(x, t)=b \sin (k x-\omega t)$, yielding the no-slip boundary condition

$\boldsymbol{u}(x+\delta(x, t), y=0)=\frac{\partial \delta}{\partial t} \boldsymbol{e}_{x}=-b \omega \cos (k x-\omega t) \boldsymbol{e}_{x}$.

For a longitudinal wave, the swimming velocity is in the same direction as the traveling wave.

The direction of swimming of Taylor's sheet can be understood on the basis of the following simple arguments. Let us consider a sheet deforming as a sine wave propagating to the right (figure 3). Consider the vertical displacements occurring along the sheet as a result of the propagating wave. During a small interval of time, the original wave (figure 3, thick blue line) has moved to the right (figure 3, thin blue line), resulting in vertical displacements given by a profile which is $\pi / 2$ out of phase with the shape of the sheet. Indeed, wherever the sheet has a negative slope, the material points go up as the wave progresses to the right, whereas everywhere the sheet has a positive slope, the material points go down as a result of the wave. The resulting distribution of vertical velocity along the sheet is illustrated in figure 3 by the black dashed line and vertical arrows. This velocity profile forces the surrounding fluid, and we see that the fluid acquires vorticity of alternating sign along the sheet (illustrated by the curved red arrows in figure 3). The vorticity is seen to be positive near the sheet crests, whereas it is negative near the sheet valleys. The longitudinal flow velocities associated with this vorticity distribution allow us to understand the swimming direction. In the case of positive vorticity, the velocity of the induced vortical flow is to the left at the wave crest, which is the current position of the sheet. In the case of negative vorticity, the induced flow velocity is to the left in the valleys, which is also where the sheet is currently located. As a consequence, the longitudinal flow field induced by the transverse motion of the sheet leads to flow contributions which are to the left in all cases, and the sheet is seen to swim to the left (straight red leftward arrows). Note that if the sheet were not free to move, then it would create an external flow field that cancels the sheet-induced flow, and the net flow direction would therefore be to the right - the sheet acts as a pump.

There are many generalizations to Taylor's 1951 calculation. Taylor himself considered the more realistic geometry of an infinite cylinder with a propagating transverse wave [95]. In this case, there is a new length scale $a$, the radius of the cylinder, and the calculation is organized as a power series in $b / a$ rather than $b k$. In the limit $a k \rightarrow 0$, the swimming velocity has the same form as the planar sheet [95]. With a cylinder, we can study truly three-dimensional deformations of a filament, such as helical waves. A helical wave can be represented by the superposition of two linearly polarized transverse waves, with perpendicular polarizations and a phase difference of $\pi / 2$. If these waves have the same amplitude, speed and wavelength, then the swimming velocity is twice the velocity for a single wave. Although the hydrodynamic force per unit wavelength acting on the waving filament vanishes, there is a non-vanishing net hydrodynamic torque per unit wavelength, which is ultimately balanced by the counterrotation of the head of the organism [95].

The Taylor sheet calculation may also be extended to finite objects. For example, to model the locomotion of ciliates such as Opalina and Paramecium, Lighthill introduced the 'envelope model,' in which the tips of the beating cilia that cover the cell body are represented by propagating surface waves [96-98]. Perhaps the simplest version of the envelop model is the two-dimensional problem of an undulating circle in the plane, which may equivalently be viewed as an infinite cylinder with undulations traveling along the circumferential direction [98]. Recall that a rigid infinite cylinder towed through liquid at zero Reynolds number suffers from the Stokes paradox: there is no solution to the Stokes equations that 
satisfies the no-slip boundary condition at the cylinder surface and has finite velocity at infinity $[60,66,99]$. The undulating cylinder does not suffer from the Stokes paradox, since the total force on the cylinder is zero. The problematic solutions that lead to the Stokes paradox are the same ones that lead to a net force, as well as a diverging kinetic energy, and are therefore eliminated in the swimming problem [98]. Furthermore, and in contrast with the Taylor sheet problem where the swimming speed emerges self-consistently, the condition of vanishing force is required to determine the swimming speed of the undulating cylinder.

In three dimensions, the swimming speed is also determined by the condition of vanishing total force $[96,97]$, but since the solution to the problem of towing a sphere with an external force is well behaved, we may also consider solutions with non-zero force. These solutions must be well behaved if we are to apply the reciprocal theorem of section 3 , which gives perhaps the shortest route to calculating the swimming speed [72]. We can also gain additional insight into why the swimming speed for a prescribed deformation of the surface is independent of viscosity. Using the linearity of Stokes flow, at any instant we may decompose the flow field generated by the swimmer into a 'drag flow' and a 'thrust flow,' $\boldsymbol{v}=\boldsymbol{v}_{\mathrm{d}}+\boldsymbol{v}_{\mathrm{t}}$ [3]. The drag flow is the flow induced by freezing the shape of the swimmer and towing it at velocity $\boldsymbol{U}$ with a force $\boldsymbol{F}$, to be determined. The thrust flow is the flow induced by the swimmer's motion at that instant when it is prevented from moving by an anchoring force $\boldsymbol{F}_{\text {anchor }}$, which is determined by the shape and the rate of change of shape of the swimmer at that instant. Superposing the two flows, and adjusting $\boldsymbol{F}$ to cancel

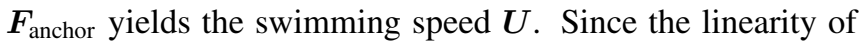
Stokes flow implies that both $\boldsymbol{F}$ and $\boldsymbol{F}_{\text {anchor }}$ depend linearly on viscosity, the swimming velocity $\boldsymbol{U}$ does not depend on viscosity. Note that the same conclusion follows from an examination of the reciprocal theorem formula, equation (10).

Finally, in the sheet calculation, it is straightforward to include the effects of inertia and show that if flow separation is disregarded, the swimming speed decreases with Reynolds number, with an asymptotic value at high Reynolds number of half the value of Taylor's result (23) [100-102]. At zero Reynolds number, the effect of a nearby rigid wall is to increase the swimming speed as the gap between the swimmer and the wall decreases, for prescribed waveform [100]. However, if the swimmer operates at constant power, the swimming speed decreases as the gap size decreases [100].

\subsection{Local drag theory for slender rods}

All the calculations of the previous subsection are valid when the amplitude of the deflection of the swimmer is small. These calculations are valuable since they allow us to identify qualitative trends in the dependence of the swimming velocity on geometric and, as we shall see in section 8 , material parameters. However, since real flagella undergo large-amplitude deformations, we cannot expect models based on small-amplitude deformations to give accurate results. Fortunately, we may develop an alternative approximation that is valid for large deformations by exploiting the fact that real

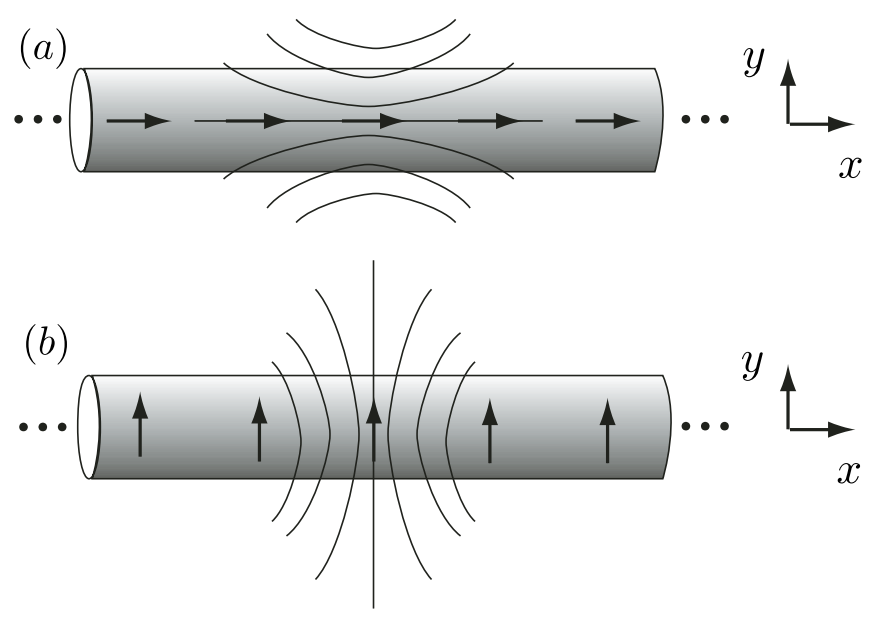

Figure 4. Model for a rod subject to an external force along the rod $(a)$ or perpendicular to the rod $(b)$. The arrows represent stokeslets. Only the flow field of the central stokeslet is shown. Each stokeslet induces a similar flow field that helps push the rest of the rod along.

flagella are long and thin. The idea is to model the flow induced by a deforming flagellum by replacing the flagellum with a line of singular solutions to Stokes flow of appropriate strength. In this subsection and the following subsection we develop these ideas, first in the simplest context of local drag theory, also known as resistive force theory, and then using the more accurate slender-body theory.

To introduce local drag theory, we develop an intuitive model for calculating the resistance matrix of a straight rigid rod of length $L$ and radius $a$. Our model is not rigorous, but it captures the physical intuition behind the more rigorous theories described below. Suppose the rod is subject to an external force $\boldsymbol{F}_{\text {ext }}$. Suppose further that this force is uniformly distributed over the length of the rod with a constant force per unit length. Our aim is to find an approximate form for the resistance matrix, or equivalently, the mobility matrix, with errors controlled by the small parameter $a / L$. To this end, we replace the rod with $N$ stokeslets equally spaced along the $x$-axis, with positions $\boldsymbol{x}_{j}=(j L / N, 0,0)$. Each stokeslet represents the leading-order far field flow induced by a short segment of the rod. According to our assumption of uniformly distributed force, the strength of each stokeslet is $\boldsymbol{F}_{\text {ext }} / N$. If there were no hydrodynamic interactions among the different segments of the rod, then each segment would move with velocity $\boldsymbol{u}=\boldsymbol{F}_{\text {ext }} / \xi_{\text {seg }}$, where $\xi_{\text {seg }} \propto \eta a$ is the resistance coefficient of a segment. Instead, the motion of each segment induces a flow that helps move the other segments along (figure 4).

To calculate the flow $\boldsymbol{u}_{j}$ induced by the $j$ th segment, we use equation (7), where

$$
\boldsymbol{u}_{j}(\boldsymbol{x})=\frac{1}{8 \pi \eta\left|\boldsymbol{x}-\boldsymbol{x}_{j}\right|}\left(\mathbf{1}+\boldsymbol{e}_{x} \boldsymbol{e}_{x}\right) \cdot\left(\boldsymbol{F}_{\mathrm{ext}} / N\right) .
$$

Since each segment moves with the local flow, we identify the velocity of the $i$ th segment with the superposition of the flow induced by the force $\boldsymbol{F} / N$ at $\boldsymbol{x}_{i}$ and the flows induced by the forces at all the other segments

$$
\boldsymbol{u}\left(\boldsymbol{x}_{i}\right)=\boldsymbol{F}_{\mathrm{ext}} / \xi_{\mathrm{seg}}+\sum_{j \neq i} \boldsymbol{u}_{j}\left(\boldsymbol{x}_{i}\right)
$$


Since $N \gg 1$, we replace the sum over $i$ by an integral, noting that the density of spheres is $L / N$, and taking care to exclude a small segment around $x_{i}$ from the region of integration

$\boldsymbol{u}\left(\boldsymbol{x}_{i}\right)=\frac{\boldsymbol{F}_{\mathrm{ext}}}{\xi_{\mathrm{seg}}}+\frac{1}{8 \pi \eta} \int^{\prime} \frac{1}{\left|x_{i}-x\right|}\left(\mathbf{1}+\boldsymbol{e}_{x} \boldsymbol{e}_{x}\right) \cdot \boldsymbol{F}_{\mathrm{ext}} \frac{\mathrm{d} x}{L}$,

where the prime on the integral indicates that the region of integration is from $-L / 2$ to $L / 2$ except for the interval around $x_{i}$ with size of order $a$. Evaluation of the integral yields

$$
\boldsymbol{u}\left(\boldsymbol{x}_{i}\right)=\frac{\boldsymbol{F}_{\mathrm{ext}}}{\xi_{\mathrm{seg}}}+\frac{1}{4 \pi \eta} \ln \left(\frac{L}{4 a}\right)\left(\mathbf{1}+\boldsymbol{e}_{x} \boldsymbol{e}_{x}\right) \cdot \frac{\boldsymbol{F}_{\mathrm{ext}}}{L},
$$

where we have disregarded end effects by assuming $\left|x_{i}\right| \ll L$. Keeping only the terms which are leading order in $\ln (L / a)$, and using the fact that $\boldsymbol{u}\left(x_{i}\right)$ is constant for a rigid rod, we find

$$
\boldsymbol{u}=\frac{\ln (L / a)}{4 \pi \eta}\left(\mathbf{1}+\boldsymbol{e}_{x} \boldsymbol{e}_{x}\right) \cdot \boldsymbol{f}_{\mathrm{ext}},
$$

where $f_{\text {ext }}=\boldsymbol{F}_{\text {ext }} / L$ is the externally imposed force per unit length. In our model, the only forces acting between any pair of segments is the hydrodynamic force: there are no internal cohesive forces. Therefore, drag per unit length $f=-f_{\text {ext }}$, and

$$
f_{\perp}=-\xi_{\perp} u_{\perp}, \quad f_{\|}=-\xi_{\|} u_{\|},
$$

where $\perp$ and $\|$ denote the components perpendicular and parallel to the $x$-axis, respectively, and $\xi_{\perp}=2 \xi_{\|}=$ $4 \pi \eta / \ln (L / a)$. Once again, we encounter the anisotropy already mentioned for slender bodies (section 3.2) and stokeslets (section 3.3) that is necessary for drag-based thrust (section 4.3).

In our derivation of equation (31), we assumed zero deformation since the filament was straight. Turning now to deformed filaments, suppose that the filament is gently curved, $\kappa a \ll 1$, where $\kappa^{2}=\left|\partial^{2} r / \partial s^{2}\right|^{2}$, and $\boldsymbol{r}(s)$ gives the position of the centerline of the filament with arclength coordinate $s$. In the limit of very small curvature, it is reasonable to assume that the viscous force per unit length acting on the curved filament is the same as the viscous force per unit length acting on a straight rod of the same length. Since local drag theory is an expansion in powers of $1 / \ln (L / a)$, it is valid for filaments that are 'exponentially thin.' That is, to make $1 / \ln (L / a)$ of order $\epsilon$ with $\epsilon \ll 1$, we need $a / L \sim \exp (-1 / \epsilon)$. In section 5.3, we introduce slender-body theory, which has the advantage of being accurate for thin $\left(a / L \sim \epsilon^{p}\right)$ rather than exponentially thin filaments.

Slender-body theory also more accurately captures the hydrodynamic interactions between distant parts of a curved filaments. To see the limitations of the resistive force theory coefficients of equation (31), consider a rigid ring of radius $R$ and rod diameter $2 a$, falling under the influence of gravity in a very viscous fluid. Suppose the plane of the ring is horizontal. Compare the sedimentation rate of the ring with that of a horizontal straight rod with length $L=2 \pi R$. In both cases, each segment of the object generates a flow which helps push the other segments of the object down. But the segments of the ring are closer to each other, on average, and therefore the ring falls faster. Using the coefficients of local drag theory from equation (31) would lead to the same sedimentation rate for both objects. This argument shows the limitations of our local drag theory. One way to improve our theory is to use a smooth distribution of stokeslets and source dipoles to make a better approximation for the flow induced by the motion of the rod. Applying this approach to a sine wave with wavelength $\lambda$, Gray and Hancock found $[31,32]$

$$
\begin{gathered}
\xi_{\|}=\frac{2 \pi \eta}{\ln (2 \lambda / a)-\frac{1}{2}}, \\
\xi_{\perp}=2 \xi_{\|} .
\end{gathered}
$$

In [12], Lighthill refined the arguments of [31] and gave more accurate values for $\xi_{\perp}$ and $\xi_{\|}$. Despite the limitations of local drag theory, we will see that it is useful for calculating the shapes of beating flagella and the speeds of swimmers.

In the rest of this section, we describe some of the applications of local drag theory to the problem of swimmers with prescribed stroke. To keep the formulae compact, we work in the limit of small deflections, although local drag theory is equally applicable to thin filaments with large deflections. Consider the problem of a spherical body of radius $R_{\mathrm{b}}$ propelled by a beating filament with a planar sine wave [83]

$$
h(x, t)=b \sin (k x-\omega t) .
$$

As in our discussion of the Taylor sheet, section 5.1, we work in the frame of the swimmer. Thus, the problem is to find the flow velocity $\boldsymbol{U}$ that yields zero net force and moment on the swimmer. To simplify the discussion, we suppose that external forces and moments are applied to the head to keep it from rotating or moving in the $y$-direction. In real swimmers, there is a transverse component of the velocity and a rotation, which both play an important role in determining the swimmer's trajectory and the shape of the flagellum [103, 104].

Equation (31) gives the viscous forces per unit length acting on the filament. The total force per unit length has a propulsive component, equation (19), arising from the deformation of the filament, and also a drag component, arising from the resistance to translating the swimmer along the $x$ direction. Integrating this force per length to find the total $x$-component of force, writing the drag force on the sphere as $\xi_{0} R_{\mathrm{b}} U=6 \pi \eta R_{\mathrm{b}} U$, and balancing the force on the sphere with the force on the filament yields

$$
U=\frac{\left(\xi_{\perp}-\xi_{\|}\right) \int_{0}^{L} \dot{h} h^{\prime} \mathrm{d} x}{\xi_{\|} L+\xi_{0} R_{\mathrm{b}}}
$$

Note that only the perpendicular component of the rod velocity leads to propulsive thrust; the motion of the rod tangential to itself hinders swimming. Inserting the sinusoidal waveform (34) into equation (35) and averaging over a period of the oscillation yields

$$
\langle U\rangle=-\frac{\xi_{\perp}-\xi_{\|}}{2 \xi_{\|}} \frac{\omega k b^{2}}{1+\left(\xi_{0} R_{\mathrm{b}}\right) /\left(\xi_{\|} L\right)} .
$$

The form of equation (36) is similar to the result for a swimming sheet, equation (23); when $L \gg R_{\mathrm{b}}$ and $\xi_{\perp}=2 \xi_{\|}$, 


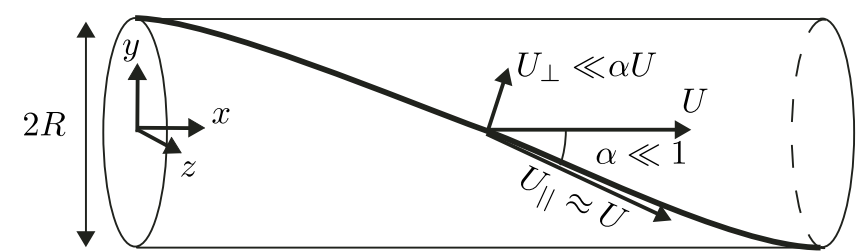

Figure 5. Forces on a helical segment pulled through a viscous fluid with speed $U$. Half of a helical pitch is shown. The helix is prevented from rotating by an external moment along $x$.

the two expressions are identical. For $L \gg R_{\mathrm{b}}$, the swimming speed is independent of $L$ for fixed $k$ and $b$, since lengthening the filament increases the drag and propulsive forces by the same amount.

Since the swimmer has finite length, we can define the hydrodynamic efficiency as the ratio of the power required to drag the swimmer with a frozen shape though the liquid at speed $U$ to the average rate of work done by the swimmer ${ }^{4}$. In other words, the efficiency is the ratio of the rate of useful working to the total rate of working. To leading order in deflection, the efficiency $e$ is given for arbitrary small deflection $h$ by

$$
e=\frac{\left(\xi_{\|} L+\xi_{0} R_{\mathrm{b}}\right) U^{2}}{\xi_{\perp} \int_{0}^{L}\left\langle\dot{h}^{2}\right\rangle \mathrm{d} x}
$$

For the sinusoidal traveling wave (34) with $\xi_{\perp}=2 \xi_{\|}$,

$$
e=\frac{k^{2} b^{2} / 2}{1+\left(\xi_{0} R_{\mathrm{b}}\right) /\left(\xi_{||} L\right)} .
$$

For small deflections, $k b \ll 1$, and the hydrodynamic efficiency is small. Biological cells displaying large-amplitude planar deformation typically have hydrodynamic efficiencies of about $1 \%$ [3, 11, 105], which is close the value for optimally designed swimmers (see section 9).

We now turn to the case of a rotating helix, such as the flagellar filament of $E$. coli. The body of the cell is taken to be a sphere of radius $R_{\mathrm{b}}$. For simplicity, suppose the radius $R$ of the helix is much smaller than the pitch of the helix, or equivalently, that the pitch angle $\alpha$ is very small (figure 5). Expressions relevant for large amplitudes may be found in [28, 106, 107]. We also assume that the axis of the helix always lies along the $x$-axis, held by external moments along $y$ and $z$ if necessary. The helix is driven by a rotary motor embedded in the wall of the body, turning with angular speed $\Omega_{\mathrm{m}}$ relative to the body. The helix rotates with angular speed $\Omega$ in the laboratory frame, and the body must counter-rotate with speed $\Omega_{\mathrm{b}}$ to ensure the total component of torque along $x$ of the swimmer vanishes. The angular speeds are related by $\Omega_{\mathrm{b}}+\Omega=\Omega_{\mathrm{m}}$.

To calculate the force and moment acting on the helical filament, we use equation (5), which is simplified to include only components along $x$-axis:

$$
\left(\begin{array}{l}
F \\
M
\end{array}\right)=\left(\begin{array}{ll}
A & B \\
B & C
\end{array}\right) \cdot\left(\begin{array}{l}
U \\
\Omega
\end{array}\right),
$$

4 Note that the total efficiency is given by the product of the hydrodynamic efficiency and the efficiency of the means of energy transduction. where $F$ and $M$ are the external force and moment, respectively, required to pull the helix with speed $U$ and angular rotation rate $\Omega$. To leading order in $\alpha$, and with the assumption that $L \gg R_{\mathrm{b}}, R$, the resistance coefficients are approximately

$$
\begin{gathered}
A \approx \xi_{\|} L, \\
B \approx-\left(\xi_{\perp}-\xi_{\|}\right) \alpha R L, \\
C \approx \xi_{\perp} R^{2} L .
\end{gathered}
$$

These values are deduced by applying equations (31) to special cases of motion. The coefficient $A$ may be found by considering the case of a helix pulled at speed $U$ but prevented from rotating. Likewise, $C$ may be found by examining a helix that rotates about $x$ but does not translate along $x$. To find $B$, we may examine the moment required to keep a helix pulled with speed $U$ from rotating (figure 5). Or we may also examine the force required to keep a helix rotating at angular speed $\Omega$ from translating. The equivalence of these two calculations is reflected in the symmetry of the resistance matrix, which ultimately stems from the reciprocal theorem, equation (4). Note that the sign of the coupling $B$ between rotational and translational motions is determined by the handedness of the helix.

To find the swimming speed $U$ and the rate of filament rotation, we equate the external forces and moments on the filament to the forces and moments acting on the cell body,

$$
\begin{gathered}
F=-\xi_{0} R_{\mathrm{b}} U, \\
M=-\xi_{\mathrm{r}} R_{\mathrm{b}}^{3} \Omega_{\mathrm{b}},
\end{gathered}
$$

where we have introduced a resistance coefficient $\xi_{\mathrm{r}}=8 \pi$ for the rotation of the sphere. Solving for the three unknowns $U$, $\Omega$ and $\Omega_{\mathrm{b}}$, we find

$$
U \approx \alpha \frac{\xi_{\perp}-\xi_{\|}}{\xi_{\|}}\left(\frac{\xi_{\mathrm{r}}}{\xi_{\perp}}\right)\left(\frac{R_{\mathrm{b}}^{3}}{R L}\right) \Omega_{\mathrm{m}},
$$

$\Omega_{\mathrm{b}}=\Omega_{\mathrm{m}}+\mathcal{O}\left(R_{\mathrm{b}} / L\right)$ and $\Omega \approx \Omega_{\mathrm{m}}\left(\xi_{\mathrm{r}} / \xi_{\perp}\right) R_{\mathrm{b}}^{3} /\left(R^{2} L\right)$. The resistance $\xi_{0}$ of the body does not enter since we assume $L \gg R_{\mathrm{b}}$. The speed is linear in $\alpha$ since the sign of the speed is given by the handedness of the helix, which is given by the sign of $\alpha$. Note the contrast with the planar wave. If $R_{\mathrm{b}}=0$, then the velocity (45) vanishes: propulsion by means of a rotating helix requires a body. In contrast, a planar wave does not require a body for propulsion (see equation (36)). For the helical filament, the swimming speed decreases with increasing $L$ for fixed body size $R_{\mathrm{b}}$ and fixed motor speed $\Omega_{\mathrm{m}}$, since torque balance forces the filament to rotate more slowly with increasing $L$. Also, since the resistance to rotation of a helix scales as $R^{2}$, the helix rotates sufficiently faster as the radius decreases to overcome the reduced thrust force implied by the linear dependence of $B$ on $R$, leading to a speed that increases like $1 / R$ for decreasing $R$. It is important to note that the motor speed $\Omega_{\mathrm{m}}$ of a swimming bacterium is not directly observable with current techniques; typically the approach just described is used to make a prediction for the relation between observables such as $U$ and $\Omega$, which is then compared with measurements $[28,108]$. To conclude we note that for swimming using a rotating helix, a hydrodynamic efficiency can be defined with analogy with the planar case; for E. coli, this efficiency is slightly less than $2 \%$ [28]. 


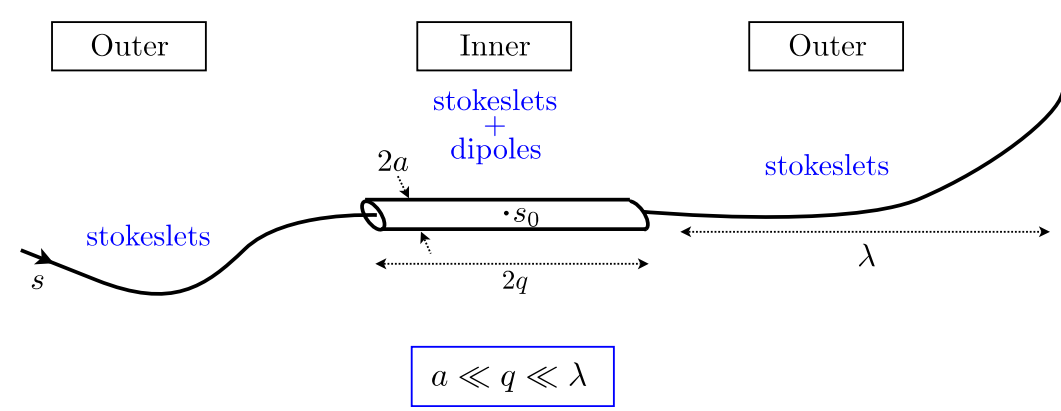

Figure 6. Lighthill's singularity construction for the slender-body theory of flagellar hydrodynamics. To compute the flow field at a point $s_{0}$ along the flagellum, Lighthill picks an intermediate length scale $q$ such that $a \ll q \ll \lambda$ and represents the flow by a distribution of stokeslests at a distance further away than $q$ from $s_{0}$ (outer problem) and a distribution of stokeslets and source dipoles within $q$ of $s_{0}$ (inner problem). The dipole strengths in the inner problem are obtained by requiring that the result be independent of $q$.

\subsection{Slender-body theory}

The local drag theory illustrated in the previous section allows an intuitive presentation of the scaling laws for flagella-based locomotion. As we saw, this theory is quantitatively correct only for exponentially slender filaments. Let $\lambda$ be the typical length scale along the flagellum on which its variations in shape occur, such as the wavelength, and $a$ the flagellum radius. The local drag theory assumes that $1 / \log (\lambda / a) \ll 1$. Since real biological flagella have aspect ratios on the order $a / \lambda \sim 10^{-2}$, an improved modeling approach is necessary $[12,109]$. The idea, termed slender-body theory and pioneered by Hancock [31], is to take advantage of the slenderness of the filaments and replace the solution for the dynamics of the threedimensional of the filament surface by that of its centerline using an appropriate distribution of flow singularities. Two different approaches to the method have been proposed.

The first approach consists of solving for the flow as a natural extension of the local theory, and approximating the full solution as a series of logarithmically small terms [110-112]. Physically, the flow field close to the filament is locally twodimensional, and therefore diverges logarithmically away from the filament because of Stokes' paradox of two-dimensional flows [62]. The flow far from the filament is represented by a line distribution of stokeslets of unknown strengths, and diverges logarithmically near the filament due to the line integration of the stokeslet terms which behave like $1 / r$ in the near field. Matching these two diverging asymptotic behaviors allows the determination of the stokeslet strengths order by order as a series of terms of order $1 /(\log \lambda / a)^{n}$. The leadingorder term in this series, of order $1 / \log (\lambda / a)$, is the local drag theory, and gives a stokeslet distribution proportional to the local velocity. The next order term is in general nonlocal and provides the stokeslet strength at order $1 /(\log \lambda / a)^{2}$ as an integral equation on the filament shape and velocity. Terms at higher order can be generated in a systematic fashion [110-112]. This approach to slender-body hydrodynamics is the logical extension to the local drag theory, and all the terms in the expansion can be obtained analytically which makes it appealing. However, the major drawback to this approach is that each term in the expansion is only smaller than the previous term by a factor $1 /(\log \lambda / a)$, so a large number of terms is necessary in order to provide an accurate model for the flow.
A second approach, asymptotically more accurate but technically more involved, consists of bypassing the logarithmically converging series by directly deriving the integral equation satisfied by the unknown distribution of singularities along the filament. Such an approach has been successfully implemented using matched asymptotics [113] or uniform expansions [114], and leads to results accurate at $\operatorname{order} a / \lambda$. This approach is usually preferable since instead of being only logarithmically correct it is algebraically correct. This improved accuracy comes at a price, however, and at each instant an integral equation must be solved to compute the force distribution along the filament. An improvement of the method was later proposed by accurately taking into account end effects and a prolate spheroidal cross-section, with a relative accuracy of order $(a / \lambda)^{2} \log (a / \lambda)$ [115].

In his John von Neumann lecture, Lighthill proposed an alternative method for the derivation of such integral equations. Instead of using asymptotic expansions, he used physical arguments to derive the type and strength of the singularities located along the filament $[12,116]$. By analogy with the flow past a sphere, Lighthill first proposed that a line distribution of stokeslets and source dipoles should be appropriate to represent the flow field induced by the motion of the filament. He then demonstrated that the strength of the dipole distribution should be proportional to the stokeslets strengths using the following argument (see figure 6). Consider a location $s_{0}$ along the filament. By assumption of slenderness, it is possible to find an intermediate length scale $q$ along the filament such that $a \ll q \ll \lambda$. The flow field on the surface of the filament at the position $s_{0}$ is then the sum of the flow due to the singularities within a distance $q$ from $s_{0}$ ('inner' problem) and those further away than $q$ from $s_{0}$ ('outer' problem). Since $q \gg a$, the contribution at $s_{0}$ from the outer problem is primarily given by a line distribution of stokeslets (the source dipoles decay much faster in space). In the inner problem, Lighthill then showed that it was possible to analytically determine the strength of the dipoles to ensure that the complete solution (sum of the inner and outer problems) was independent of the value of $q$. The dipole strength is found to be proportional to the stokeslet strength, and the resulting value of the velocity of the filament at $s=s_{0}$ is given by the integral equation

$$
\boldsymbol{u}\left(s_{0}\right)=\frac{\boldsymbol{f}_{\perp}\left(s_{0}\right)}{4 \pi \eta}+\int_{\left|r_{0}-r\right|>\delta} \boldsymbol{G}\left(\boldsymbol{r}_{0}-\boldsymbol{r}\right) \cdot \boldsymbol{f}(s) \mathrm{d} s .
$$


In equation (46), $f$ is the local strength of the unknown stokeslet distribution, with dimensions of force per unit length; $G$ is the Oseen tensor from equation (7); $\delta=a \sqrt{\mathrm{e}} / 2$ the natural cutoff, regularizing the divergences arising from self-interaction; and $f_{\perp}$ represents the normal component of the stokeslet distribution, i.e. $f_{\perp}=(\mathbf{1}-\boldsymbol{t} t) \cdot f$ if $t$ is the local tangent to the filament. Note that Lighthill's slender-body analysis is less mathematically rigorous than those presented in $[113,114]$, and consequently gives results which are only valid at order $(a / \lambda)^{1 / 2}[3]$. His derivation provides, however important physical insight into a subject, the topic of flow singularities, that is usually very mathematical. The resulting integral formulation, equation (46), is relatively simple to implement numerically, and can also be used to derive 'optimal' resistance coefficients for the local drag theory (see section 5.2). His modeling approach has been extended for filament motion near a solid boundary [117-120], and an alternative approach based on the method of regularized flow singularities [121] has also been devised [122].

\section{Physical actuation}

In this section we describe common mechanisms for the generation of non-reciprocal swimming strokes. In addition to biological mechanisms such as rotating helices or beating flagella, we also describe simple mechanisms that do not seem to be used by any organism. These non-biological systems are useful to study since they deepen our understanding of the biological mechanisms. For example, the modes of an elastic rod driven by transverse oscillations at one end are useful for understanding the shape of a beating flagellum driven by motors distributed along its entire length. An important theme of this section is the fluid-structure interaction for thin filaments in viscous liquid.

\subsection{Boundary actuation}

We begin with the case of boundary actuation, in which an elastic filament is driven by a motor at its base. The rotary motor of the bacterial flagellum is a prime example of such a biological actuating device $[20,37,123,124]$. The steadystate relation between motor torque and motor speed is shown in figure 7 [37]. At low speeds, the motor torque is roughly constant; at higher speeds it decreases linearly with speed, reaching zero torque at about $300 \mathrm{~Hz}$ at $23{ }^{\circ} \mathrm{C}$. To determine the speed of the motor from the motor torque-speed relations, we use torque balance and equate the motor torque with the load torque. By the linearity of Stokes flow, the load torque is linear in rotation speed. In the experiments used to make the graph of figure 7, the flagellar filament of E. coli was tethered to a slide, and the rotation of the body was observed. A typical body of $1 \mu \mathrm{m}$ radius has a substantial resistance, leading to the steep load curve on the left of figure 7 and a correspondingly low rotation speed. A smaller load, such as that of a minicell, leads to a load curve with smaller slope, and higher rotation speed. The torque-speed characteristic $M\left(\Omega_{\mathrm{m}}\right)$ allows us to go beyond the artifice of the previous section where we calculated the swimming speed $U$ in terms of the motor speed $\Omega_{\mathrm{m}}$. Solving

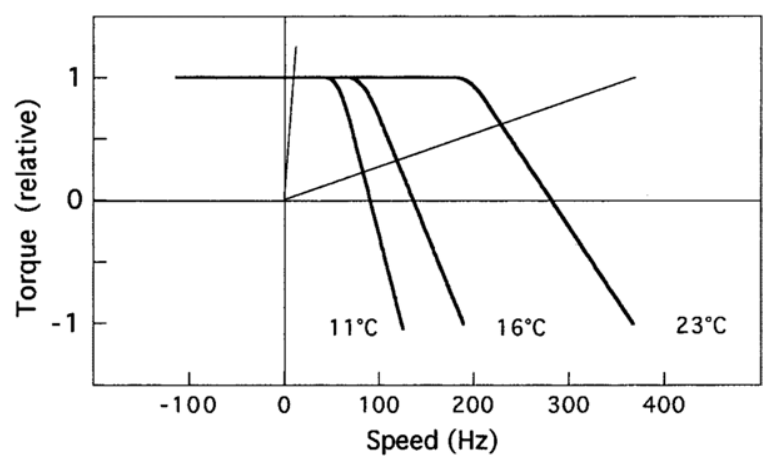

Figure 7. The torque-speed characteristic for the flagellar motor of $E$. coli for three different temperatures (bold lines). Also shown (thin lines) are the load lines for a cell body with radius of $1 \mu \mathrm{m}$ (left), and a minicell with effective radius of about $0.3 \mu \mathrm{m}$ (right). Figure reprinted from [37], courtesy of Howard Berg and by permission from Annual Reviews, copyright 2003 (doi:10.1146/annurev.biochem.72.121801.161737).

equations (39) and (43)-(44) along with $M=M\left(\Omega_{\mathrm{m}}\right)$ yields the swimming speed in terms of the geometrical parameters of the flagellum, the cell body, the drag coefficients and the properties of the motor.

In the previous section we described how a rotating helix generates propulsion. Since the flagellar filaments of $E$. coli and S. typhimurium are relatively stiff, a helical shape is necessary to escape the constraints of the scallop theorem, since a straight rod rotating about its axis generates no propulsion. Indeed, mutant $E$. coli with straight flagella do not swim [125]. If the rate of rotation of a straight but flexible rod is high enough for the hydrodynamic torque to twist the rod through about one turn, then the rod will buckle into a gently helical shape that can generate thrust [126-128]. However, the high twist modulus of the filament and the low rotation rate of the motor make this kind of instability unlikely in the mutant strains with straight flagella. On the other hand, a rotating helix with the dimensions of a flagellar filament experiences much greater hydrodynamic torque since the helical radius (micrometers) is much greater than the filament radius $(\approx 10 \mathrm{~nm})$. The handedness of the helix also breaks the symmetry of the response to the sense of rotation of the motor: counter clockwise rotation of a left-handed helix in a viscous fluid tends to decrease the pitch of a helix, whereas clockwise rotation tends to increase the pitch [129]. There is no noticeable difference between the axial length of rotating and de-energized flagella for counter-clockwise rotation [130]; calculations of the axial extension [129] based on estimates of the bending stiffness of the flagellar filament [131] are consistent with this observation. However, the hydrodynamic torque for clockwise rotation is sufficient to trigger polymorphic transformations, in which a right-handed helical state invades the left-handed state by the propagation of a front [132,133] (see also [134-137]).

Now consider the case of an elastic filament driven by a mechanism that oscillates the base of the filament in the direction normal to the tangent vector of the filament. Although we know of no organism that uses this mechanism to swim, study of this example has proven instructive. In 


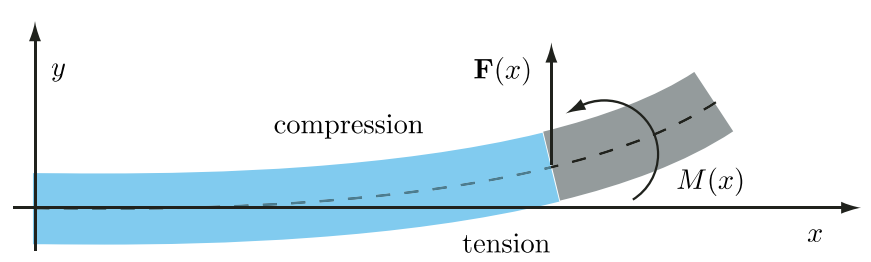

Figure 8. A bent elastic rod. The darkly shaded part of the rod exerts a force $\boldsymbol{F}(x)$ and moment $M(x)$ on the lightly shaded part through the cross-section at $x$.

early work, Machin [138] pointed out that the overdamped nature of low-Re flow leads to propagating waves of bending with exponential decay of the amplitude along the length of the filament. Since the observed beating patterns of sperm flagella typically have an amplitude that increases with distance from the head, Machin concluded that there must be internal motors distributed along the flagellum that give rise to the observed shape. This problem has served as the basis for many subsequent investigations of fluid-structure interactions in swimming [94, 104, 138-144], and has been applied to the determination of the persistence length of actin filaments [145, 146], so we now present a brief overview of its most important results.

Consider a thin rod of length $L$ constrained to lie in the $x y$-plane, aligned along the $x$-axis in the absence of external loads. We will consider small deflections $h(x)$ from the straight state. When the rod is bent into a curved shape, the part of the rod on the outside of the curve is under tension, while the part of the rod on the inside of the curve is under compression (figure 8). Therefore, the section of the rod to the right of $x$ exerts a moment $M=A \kappa(x) \approx A \partial^{2} h / \partial x^{2}$ on the section to the left of $x$, where $A$ is the bending modulus and $\kappa(x)$ is the curvature of the rod at $x$ [147]. Working to first order in deflection, balance of moments on an element of length $\mathrm{d} x$ of the rod implies

$$
\frac{\partial M}{\partial x}+F_{y}=0
$$

or $F_{y}=-A \partial^{3} h / \partial x^{3}$ for the $y$-component of the force exerted through the cross-section at $x$. Thus, if the rod has a deflection $h(x)$, then an elastic force $f_{y} \mathrm{~d} x=\left(\partial F_{y} / \partial x\right) \mathrm{d} x=$ $-\left(A \partial^{4} h / \partial x^{4}\right) \mathrm{d} x$ acts on the element of length $\mathrm{d} x$ at $x$. Balancing this elastic force with the transverse viscous force from resistive force theory yields a hyper-diffusion equation

$$
\xi_{\perp} \frac{\partial h}{\partial t}=-A \frac{\partial^{4} h}{\partial x^{4}} .
$$

The shape of the rod is determined by solving equation (48) subject to the appropriate boundary conditions, which are typically zero force and moment at the far end, $x=L$. At the near end, common choices are oscillatory displacement $h(0, t)=b \cos (\omega t)$ with clamping $\partial h /\left.\partial x\right|_{x=0}=0$, or oscillatory angle $\partial h /\left.\partial x\right|_{x=0}=\theta \cos (\omega t)$ with $h(0, t=0)$, where $b / L \ll 1$ and $\theta \ll 1[138,140]$.

The appearance of $\partial h / \partial t$ in equation (48) causes the breakdown of kinematic reversibility: even for a reciprocal actuation such as $h(0, t)=b \cos (\omega t)$, equation (48) implies that the rod shape is given by damped bending waves.
Physically, the breakdown of kinematic reversibility occurs because flexibility causes distant parts of the rod to lag the motion of the rod at the base. We saw in section 3 that zero- $R e$ flow is effectively quasistatic since the diffusion of velocity perturbations is instantaneous. When the filament is flexible, the time it takes for perturbations in shape to spread along the rod scales as $\xi_{\perp} L^{4} / A$. Since the shape of the rod does not satisfy kinematic reversibility, the flow it induces does not either, and a swimmer could therefore use a waving elastic rod to make net progress [104].

The wavelength and the decay length of the bending waves is governed by a penetration length, $\ell=\left[A /\left(\xi_{\perp} \omega\right)\right]^{1 / 4}$. Sometimes this length is given in the dimensionless form of the 'sperm number', $\mathrm{Sp}=L / \ell=L\left(\omega \xi_{\perp} / A\right)^{1 / 4}$. If the rod is waved rapidly, the penetration length is small $\ell \ll L$ and propulsion is inefficient since most of the filament has small defection and contributes drag but no thrust. For small frequencies, we get $L \ll \ell$ so the rod is effectively rigid; kinematic reversibility is therefore restored and there is no average propulsion in this limit. Thus, we expect the optimum length for propulsion is $\ell \approx L$, since at that length much of the rod can generate thrust to compensate the drag of pulling the filament along $x[140,104]$. Note that our discussion of flexibility may be generalized to other situations; for example, the deformation of a flexible wall near a swimmer is not reversible, leading to a breakdown of the scallop theorem even for a swimmer that has a reciprocal stroke [79]. In this case the average swimming velocity decays with a power of the distance from the wall, and therefore this effect is relevant in confined geometries.

An interesting variation on the Machin problem is to rotate a rod which is tilted relative to its rotation axis. If the rod is rigid, then it traces out the surface of a cone. But if it is flexible, then the far end will lag the base, and the rod has a helical shape. As long as the tilt angle is not too small, this shape may be determined without considering effects of twist $[148,149]$. If the driving torque rather than speed is prescribed, there is a transition at a critical torque at which the shape of the rod abruptly changes from gently helical to a shape which is much more tightly wound around axis of rotation, with a corresponding increase in thrust force [150-152].

\subsection{Distributed actuation}

We now consider distributed actuation, in which molecular motors are distributed along the filament. Eukaryotic flagella and cilia use distributed actuation. Figure 9 shows a crosssection of the axoneme, the core of a eukaryotic flagellum. As mentioned in the introduction, the axoneme consists of nine microtubule doublets spaced along the circumference of the flagellum, with two microtubules running along the center. In this review we restrict our attention to the case of planar beating, although many sperm flagella exhibit helical beat patterns, and nodal cilia have a twirling, rotational beat pattern $[153,154]$. The bending of the eukaryotic flagellum arises from the relative sliding of neighboring microtubule doublets [155-158]. The sliding is caused by the action of ATP-driven dynein motors, which are spaced every $24 \mathrm{~nm}$ 


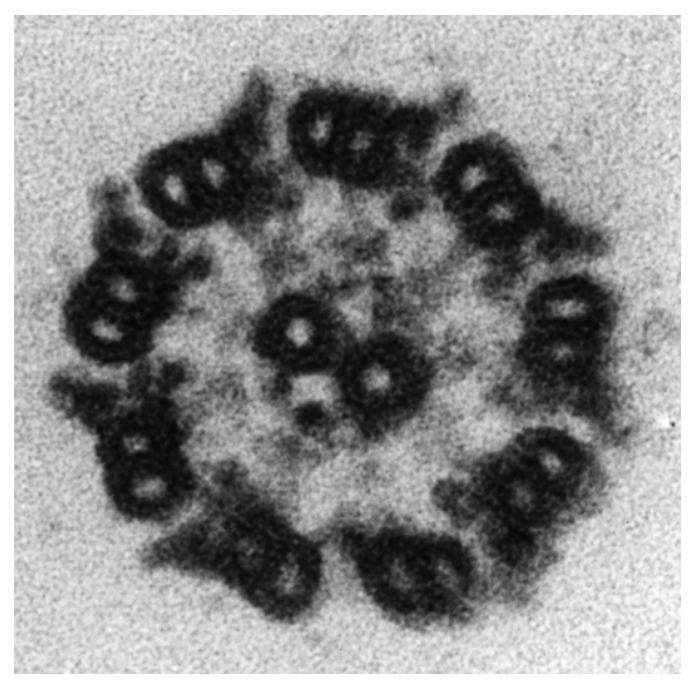

Figure 9. Cross-section section of an axoneme from wild type Chlamydomonas, courtesy of Steve King. The diameter is approximately $200 \mathrm{~nm}$. Note that the plane containing the active motors is perpendicular to the plane defined by the beating filament.

along the microtubles [92]. Since the relative sliding of the microtubules at the end near the head is restricted [159], and since each microtubule doublet maintains its approximate radial position due to proteins in the core of the flagellum, the filament must bend when the motors slide microtubule doublets. For example, in figure 10, motors have slid the lower doublet to the right relative to the upper doublet.

A simple approach to understanding how the sliding of the microtubules generates propulsion is to prescribe a density of sliding force and deduce the shape of the flagellum and therefore the swimming velocity from force and moment balance. This approach is taken in [94, 160], where the effects of viscosity and viscoelasticity are studied. A more complete model would account for how the coordination of the dynein motors arises [92,93]. Over the years, several different models for this coordination have been suggested. Since sea urchin sperm flagella continue to beat when they have been stripped of their membranes with detergent [161], it is thought that the motor activity is not coordinated by a chemical signal but instead arises spontaneously via the mechanics of the motors and their interaction $[162,163]$. A detailed discussion of molecular motors and the different mechanisms that have been put forth for controlling the beat pattern would take us too far afield [164-168]. Instead we review regulation by load-dependent motor detachment rate as presented by RiedelKruse and collaborators, who showed that this mechanism is consistent with observations of the flagellar shape [159].

The first step is to simplify the problem by considering planar beating and projecting the three-dimensional flagellum of figure 9, with nine internal circumferential filaments, onto a planar flagellum with only two internal filaments, such as that shown in figure 10. Since the lateral spacing between the two filaments is a constant, $a$, the amount that the top filament slides by the bottom is given by

$$
\Delta(x)=\Delta_{0}+a[\theta(x)-\theta(0)],
$$

where $\Delta_{0}$ is the sliding displacement at the base of the flagellum where $x=0$, and $\theta(x)$ is the angle the tangent of the flagellum centerline makes with the $x$-axis. In figure 10, $\Delta_{0}=0$, and $\Delta<0$ except at either end of the flagellum. We will forbid sliding at the base, but it is straightforward to allow such sliding in our model, and measurements of the shape of bull sperm flagella suggest that $\Delta_{0} \approx 55 \mathrm{~nm}$ [159]. Equation (49) in the case $\Delta_{0}=0$ is most easily deduced when the two filaments form the arcs of concentric circles, but it holds for more general curved shapes as well. Also, we will work in the limit of small deflection, but equation (49) is valid even for large deflection.

Now consider the forces between the two internal filaments. Let $f(x, t)$ denote the force per unit length along $x$ that the bottom filament exerts on the top filament. This force per unit length could arise from passive resistance to sliding as well as motors. The internal moment, $M$, acting on a crosssection has a contribution from bending and an additional piece from $f$

$$
M=A \frac{\partial^{2} h}{\partial x^{2}}-a \int_{x}^{L} f \mathrm{~d} x .
$$

Moment balance on an element of the flagellum yields the transverse force acting on a cross-section, $F_{y}=-A \partial^{3} h /$ $\partial x^{3}-a f$, which with force balance yields the equation of motion for the filament as

$$
\xi_{\perp} \frac{\partial h}{\partial t}=-A \frac{\partial^{4} h}{\partial x^{4}}-a \frac{\partial f}{\partial x} .
$$

For example, if the motors are not active and there is only elastic resistance to sliding, $f=-K \Delta$, then the shape equation is

$$
\xi_{\perp} \frac{\partial h}{\partial t}=-A \frac{\partial^{4} h}{\partial x^{4}}+K a^{2} \frac{\partial^{2} h}{\partial x^{2}} .
$$

Even if the bending stiffness $A$ vanishes, the elastic resistance to sliding provides a restoring force that tends to restore the straight state. More generally, the passive resistance will have a viscous as well as an elastic component: $f=-K \Delta-\lambda \dot{\Delta}$.

Now consider an active component to $f$. As in the case of the bacterial rotary motor, the speed of a dynein motor is thought to decrease with load. One end of the dynein is strongly attached to a microtubule doublet, while the other end randomly attaches to and detaches from the neighboring doublet. The motor only does work when attached. The proposal of $[92,93,163]$ is that oscillations in force arise spontaneously since the detachment rate increases with load. To see that a load-dependent detachment rate leads to positive feedback, consider a collection of motors sliding one filament past another. If there is a perturbation in which the sliding rate increases, then the load on each individual motor must decrease, according to the force-speed motor characteristic. On the other hand, the decrease in force per motor leads to an reduction in the detachment rate, which in turn leads to more attached motors and a greater total sliding force, and ultimately, an increase in sliding velocity. Working in the frequency domain, we may define a susceptibility $\chi$ via $\tilde{f}=-\chi(\omega) \tilde{\Delta}$. For the passive elements mentioned above, 


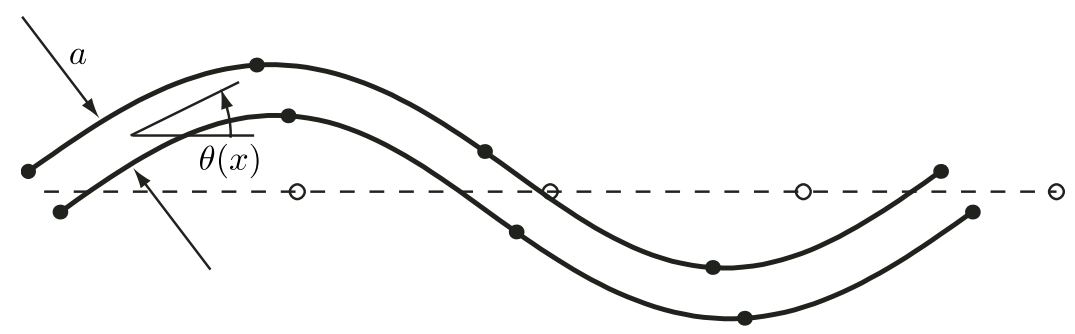

Figure 10. Simple model for a planar flagellum actuated by sliding filaments. The dotted line is the center of the flagellum when it is straight. The solid lines represent the two microtubule doublets that the active motors slide relative to each other. The two solid curves and the dotted line all have the same length; the dots divide each curve into quarters as measured along the contour.

$\chi=K+\mathrm{i} \omega \lambda$. A simple quantitative model with loaddependent detachment rate leads to a susceptibility $\chi$ that can have a negative effective elastic constant or a negative effective drag term [159]. By using equation (51) to relate the shape of the beating flagellum to the susceptibility $\chi$, Riedel-Kruse and collaborators showed that the passive components of $\chi$ were small compared with the active components; in other words, the forces and moments induced by the motors are balanced by viscous drag and bending forces, rather than internal resistance to sliding. Since the oscillations arise spontaneously in this model, the calculation of the beating filament shape amounts to solving an eigenvalue problem $[92,93]$.

We close this section by noting that there are several other distinct means of actuation. The bacterium Spiroplasma has an internal helical ribbon that is thought to undergo contractions that in turn cause the body to change shape. The shape change amounts to the propagation of kinks down the body, where the kinks are generated by a change in the handedness of the cell body [49,169]. Spirochetes such as the Leptospiracae have internal flagella that wrap around the periplasmic space between the cell body and an outer sheath. The flagellar filaments emerge from motors at either pole of the cell, but the rotating flagella distort the body in a nonreciprocal way that leads to locomotion [170,171]. These swimmers have inspired recent table-top experiments in which rigid superhelices sediment in a very viscous fluid [172]. The helical shape causes the superhelices to rotate as they fall. An important finding of [172] is that for tightly coiled superhelices the local drag theory theory is inadequate.

\section{Hydrodynamic interactions}

\subsection{Interactions between cells}

Microorganisms swimming in viscous fluids typically do so within semi-dilute or dense cell populations. As an organism swims, it sets up a flow which is felt by the cells nearby. These flows can affect the dynamics at the level of the entire population. For example, spermatozoa involved in human reproduction may swim in population sizes as high as millions of cells [2]. Bacterial suspensions are known to display so-called 'bacterial turbulence,' where large-scale intermittent motion in the forms of swirls and jets is set up when the cells become sufficiently concentrated [173-181]. Even for small numbers of cells, hydrodynamic interactions are suspected to play an important role, in particular in reproduction. Such is the case for spermatozoa of the wood mouse which aggregate, and thereby swim faster [182]. The pairing of the opossum spermatozoa enable them to swim more efficiently in very viscous fluids [183]. Fishfly spermatozoa cluster in dense bundles for similar reasons [184]. Recently, sea urchin sperm cells were observed to arrange into periodic vortices [185]. Finally, it has been conjectured that flows driving the aggregation of the algae Volvox at surfaces enhance the probability of fertilization [186].

Let us consider the interactions between two swimming cells. The flow field created by the first organism (cell A) will in general lead to two types of passive hydrodynamic interactions with its neighbor (cell B). First, cell B will feel the velocity field created by cell $\mathrm{A}$, and will be carried along by this flow as a result. In addition, cell $\mathrm{B}$ will also feel the gradients in the velocity field created by cell $\mathrm{A}$, which tend to change the orientation of $\mathrm{B}$ and thereby affect its future swimming direction.

The first type of hydrodynamic interaction can be intuitively understood by considering the far-field flow created by a swimming cell (figure 11). As discussed in section 3.3, since a cell is force-free, the velocity in the far field is typically a force dipole, decaying as $1 / r^{2}$. Two different types of force dipoles can in general arise, leading to significantly different physical pictures. Consider a microorganism with an elongated body and let $e$ be a unit vector attached to the cell and pointing along the swimming direction, which is also along the elongated direction of the cell. Usually, the force dipole $\boldsymbol{p}$ will also be in the swimming direction, $\boldsymbol{p}=p \boldsymbol{e}$, but it can have either a positive or negative sign. Cells for which $p>0$ are called 'pushers,' and include typical swimming spermatozoa, or bacteria such as E. coli. Pushers repel fluid from the body along the long axis, and draw fluid in to the sides (figure 11(a); the red solid arrows represent local forcing from the cell on the surrounding fluid and the blue dotted arrows represent the flow direction in the fluid). Cells with $p<0$ are called 'pullers,' the prototypical example of which is the alga Chlamydomonas (see also the artificial swimmer in [187]). Pullers draw fluid in along the elongated direction, and push fluid out from the sides (figure 11(b)). Note that Caulobacter cells alternate between pusher and puller mode when they reverse the direction of flagellar rotation. Mathematically, the flow induced at a distance $r$ from the dipole $\boldsymbol{p}=p \boldsymbol{e}$ is given by

$$
\boldsymbol{u}(\boldsymbol{r})=\frac{p}{8 \pi \eta r^{3}}\left[3 \cos ^{2} \theta-1\right] \boldsymbol{r},
$$




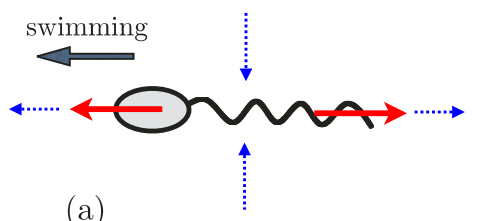

(a)

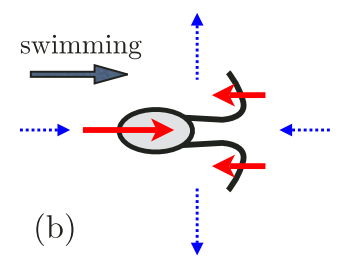

(c)
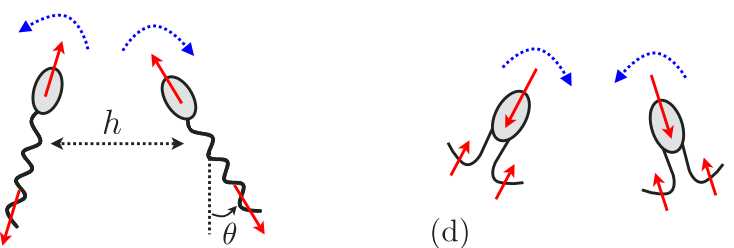

(d)

Figure 11. The flow field created by a swimmer at low Reynolds number. (a) Cells which are pushers have a positive force dipole ( $p>0$, see text) and induce a flow field directed away from the cells along their swimming direction (repulsion) and a flow field directed toward the cells along their sides (attraction) (red solid arrows represent local forcing from the cell on the surrounding fluid, and blue dotted arrows represent the flow direction in the surrounding fluid); (b) Pullers have a negative force dipole $(p<0)$, inducing an attractive flow field along their swimming direction and a repulsive flow field along their sides (in figures $(a)$ and $(b)$ the swimming direction of the cell are indicated with a thick gray arrow); $(c)$ two pushers on a converging course reorient each other, tending toward a configuration with cells parallel and swimming side-by-side ( $h$ is the separation distance between the cells, and $\theta$ is the angle between the cell direction and the direction normal to their separation); (d) two pullers on a diverging course reorient each other, tending toward a configuration in which the cells are antiparallel, swimming away from each other.

where $\theta$ is the angle between the dipole direction, $e$, and the flow position, $r$. Physically, the dipole strength scales as $|p| \sim \eta U L^{2}$ where $U$ is the swimming speed of the cell, and $L$ its typical length scale. From equation (53), we see that two similar cells swimming side-by-side $(\theta=\pi / 2)$ experience a relative velocity scaling as $\Delta u_{\text {side }} \sim-p / \eta r^{2}$. Therefore two side-by-side pushers attract each other, while two side-by-side pullers repel each other [188]. Conversely, the relative velocity of two swimmers aligned along their swimming direction, with one following the other $(\theta=0)$, scales as $\Delta u_{\text {aligned }} \sim p / \eta r^{2}$, with the opposite sign of attraction or repulsion.

The second effect of the hydrodynamic interaction between two cells is reorientation due to velocity gradients. The vorticity field induced by the cell is found by taking the curl of equation (53),

$$
\boldsymbol{\omega}=\nabla \times \boldsymbol{u}=\frac{3 p}{4 \pi \eta} \frac{(\boldsymbol{e} \cdot \boldsymbol{r})(\boldsymbol{e} \times \boldsymbol{r})}{r^{5}} .
$$

A sphere subject to this flow will rotate at a rate of half the vorticity, $\Omega=\frac{1}{2} \boldsymbol{\omega}$, to leading order in the ratio of the sphere size and the separation of the sphere from the microorganisms inducing the flow [61]. For interacting cells, which are typically not spherical, an additional component for $\Omega$ arises from the symmetric part of the rate of strain, $\boldsymbol{E}=\frac{1}{2}\left[(\nabla \boldsymbol{u})+(\nabla \boldsymbol{u})^{\mathrm{T}}\right]$. Non-spherical cells tend to align with the principal axis of strain, with $\Omega \propto e \times(\boldsymbol{E} \cdot \boldsymbol{e})$. The sign of the proportionality constant and thus the sense of the rotation depends on the shape of the cells, with a positive constant for prolate cells and a negative constant for oblate cells [61,71]. As in the case of the attraction and repulsion between cells, both $\boldsymbol{\omega}$ and $\boldsymbol{E}$ change sign with $p$, and qualitatively different rotational behaviors are expected to occur for pushers versus pullers. Nearby pushers on a converging course induce flow fields on each other that reorient them in the side-by-side configuration (figure $11(c)$ ). If the cells are separated by a distance $h$, and are oriented with an angle $\theta$ with respect to the distance perpendicular to their separation, the reorientation takes place with a rotation rate $\Omega \sim-p \theta / \eta h^{3}$. In contrast, pullers induce the opposite flow field, which leads to a reorientation of the cells in the elongated direction (figure $11(d)$ ). Interestingly, for both pushers and pullers, the final configuration is one for which cells induce attracting flow fields on each other. As discussed below, similar results govern the orientation of cells near boundaries.

The results above describe the leading-order hydrodynamic interactions between cells. Higher-order effects can be considered with various levels of modeling accuracy, two of which we address here. First, there is an active component to hydrodynamic interactions. The physical reason is the following: in the flow field created by cell A, cell B induces its own disturbance flow (just as a solid body sets up a disturbance flow when located in a shear flow), which then influences the velocity and orientation of cell A. This 'reflection' is a weaker effect than the direct interaction considered above, since it decays faster in space. However it is important for artificial swimmers with no permanent dipoles [189]. Higherorder reflections can be considered in a similar manner. In addition, the flow induced by a cell is only dipolar at leading order, and includes also higher singularities that decay faster, such as source dipoles and force quadrupoles. The quadrupolar contribution is important because it dominates the pair velocity correlations, as recently demonstrated experimentally for swimming E. coli [190].

Beyond the simple physical picture presented above, a few studies have looked in detail at hydrodynamic interactions between more realistic models of swimming cells. Interactions between two cells were studied analytically and numerically for two different cases, spherical squirming (swimming motion where all surface deformation occurs tangentially to the swimmer surface) [191,192] and swimming with a single helical flagellum [193]. Detailed experiments were also carried out to study hydrodynamic interactions between the protozoan Paramecium [192]. In all cases, the far-field physical picture described above is correct, but the details of hydrodynamic interactions at short range are also important, and in many cases lead to an instability of the side-by-side configuration for pushers, and unsteady three-dimensional cell trajectories.

Beyond the dilute limit, dense cell suspensions display remarkable complex dynamics. Oriented suspensions of swimmers are found to be unstable to perturbations of long wavelength $[194,195]$, with persistence of short-range order [195], leading to nonlinear states of jets and swirls [196] similar to those observed experimentally [173-180] (see [197] for a two-dimensional study). Isotropic suspensions have also 
been found to be unstable [196]. As a result of the largescale motion in dense suspensions, swimming cells undergo a remarkable decrease in effective diffusivity [198-201].

One final important feature of cell suspensions is their rheological characteristics [202, 203]. In the limit where cells do not interact with each other hydrodynamically, the response of a cell population to an external shear can be quantified using Batchelor's theory for suspensions of force-free bodies [68]. For simplicity, let us consider spherical cells with radius $L$, described by force dipoles $p=p e$, and distributed with volume fraction $c \ll 1$ in a Newtonian fluid of viscosity $\eta$. If an external shear flow with shear rate $\dot{\gamma}$ is applied to the suspension, the effective viscosity of the population $\eta_{\text {eff }}$ differs from the background viscosity of the Newtonian fluid at leading order in $c$ by the amount

$$
\frac{\eta_{\mathrm{eff}}}{\eta}=1+\left[\frac{5}{2}+\frac{3 \tau_{\mathrm{s}}\left\langle e_{1} e_{2}\right\rangle}{4 \pi \eta \dot{\gamma}}\right] c+O\left(c^{2}\right) .
$$

In equation (55), the coefficient $5 / 2$ is the Einstein contribution to the viscosity [66], $\langle\cdots\rangle$ denotes averages over the cell population, $e_{1}$ and $e_{2}$ refer to the components of the swimming direction along the external flow and shear directions respectively, and $\tau_{\mathrm{s}}=p / L^{3}$ is the typical active stress created by the swimming cell. Physically, equation (55) states that the dissipation in the fluid is different from that in the background Newtonian solvent due to two effects. One is the passive resistance of the cells to the shear flow, and the second is the active flow created by the swimming cells. Since the direction $e$ of each cell will rotate as a result of the external shear, the effective viscosity of the population, equation (55), is expected unsteady and shear-dependent. When $\tau_{\mathrm{s}} \ll \eta \dot{\gamma}$, the viscosity is dominated by the passive (Einstein) response of the cells, whereas when $\tau_{\mathrm{s}} \gg \eta \dot{\gamma}$ the rheology is expected to be governed by active stresses. For swimming E. coli, $p \approx(0.1-1) \times 10^{-18} \mathrm{Nm}$ [204] and $L \approx 1-10 \mu \mathrm{m}$, so $\tau_{\mathrm{s}} \approx 10^{-4}-1 \mathrm{~Pa}$. In addition, anisotropy in the distribution of swimming cells (e.g. orientationally ordered state [202]) leads in general to non-isotropic pressures, and therefore normal stress differences with normal stress coefficients, $\Psi_{1}$ and $\Psi_{2}$, scaling as [205-213]

$$
\begin{aligned}
& \Psi_{1}=\frac{3 \tau_{\mathrm{s}}}{4 \pi \dot{\gamma}^{2}}\left\langle e_{1} e_{1}-e_{2} e_{2}\right\rangle c, \\
& \Psi_{2}=\frac{3 \tau_{\mathrm{s}}}{4 \pi \dot{\gamma}^{2}}\left\langle e_{2} e_{2}-e_{3} e_{3}\right\rangle c .
\end{aligned}
$$

A detailed study of the rheological characteristics of non-dilute suspensions of cells was carried out for spherical squirmers [203]. Hydrodynamic interactions give rise to time-varying cell-cell configurations, and affect therefore the rheological properties at order $O\left(c^{2}\right)$ [68].

\subsection{Interactions between cells and boundaries}

Just as other nearby cells influence the dynamics of a swimming microorganism, the presence of boundaries, and more generally confinement, has a significant impact on cell locomotion. In addition to affecting the concentration of chemical species that influence the motility of microorganisms [214], boundaries modify the hydrodynamic stresses acting on the cells, and near-wall motility is therefore both biologically and physically different from bulk motility. Biological locomotion near boundaries includes surface-associated bacterial infections [215,216], biofilm formation [217,218], spermatozoa locomotion at the uterotubual junction [2] and surface-associated behavioral change [219]. In this section, we focus on the fluid mechanics of locomotion near walls.

Four distinct aspects of cell locomotion are modified by the presence of nearby boundaries. The first is the change in the swimming speed near a wall, which was addressed theoretically by a number of studies [13, 100, 220-222]. Since viscous drag increases as a body comes closer to a boundary (see section 3.3), it might be expected that a cell would slow down. However, since the propulsion method is also dragbased, a closer look is necessary. Indeed, for the Taylor sheet geometry, we mentioned in section 5.1 that the presence of a nearby wall leads to an increase in the swimming speed for a swimmer with a prescribed waveform. Here we consider this matter further for a swimmer that has no head, and swims using planar waves on a flagellum. As was shown in equation (35), for a given waveform of the flagellum, the swimming speed of the cell is an increasing function of the ratio between the perpendicular $\left(\xi_{\perp}\right)$ and parallel $\left(\xi_{\|}\right)$drag coefficients. Both coefficients are found to increase near a wall, but $\xi_{\perp}$ increases faster than $\xi_{\|}$, leading to an increase in the ratio $\xi_{\perp} / \xi_{\|}$and therefore the swimming speed. Physically, for fixed waveform, the drag-based propulsive force generated by the flagellum increases near the wall, and so does the resistive drag on the swimmer, but the propulsion increase is stronger, and therefore the swimming speed goes up. Associated with the increase in the speed is an increase in the rate of working that the swimmer has to provide in order to maintain the same waveform near the wall $[13,100,220-223]$. If alternatively the swimmer is assumed to swim with constant power, then the presence of a boundary leads in general to a decrease in the swimming speed, resulting in a decrease in the swimming efficiency except for some special flagellar waveforms [100, 220].

The second type of wall-influence on the swimming kinematics of some microorganisms is a change in their trajectories. This is most famously the case for swimming bacteria with helical flagella, such as $E$. coli, which change their swimming trajectory from straight to circular near a surface (figure 12(a)). In an infinite fluid E. coli swims, on average, in a straight line, with a swimming speed found by balancing flagellar propulsion by drag (see section 5.2). The chiral shape of the flagella is important for propulsion generation, but since the propulsive force is axisymmetric when averaged over on period of flagella rotation, the motion occurs on a straight line. Near a wall, the chiral propulsion mechanism leads a breaking of the time-averaged axisymmetry of the propulsive force because new non-zero components arise in the motility matrix of the helix [224]. For a helix parallel to a surface, the presence of a boundary leads to a non-zero coupling between the rotation of the helix around its axis (figure $12(b)$, blue dotted arrow), and the force on 


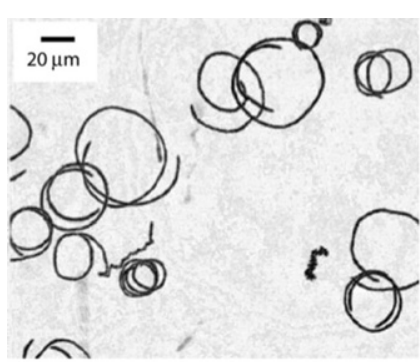

(a)

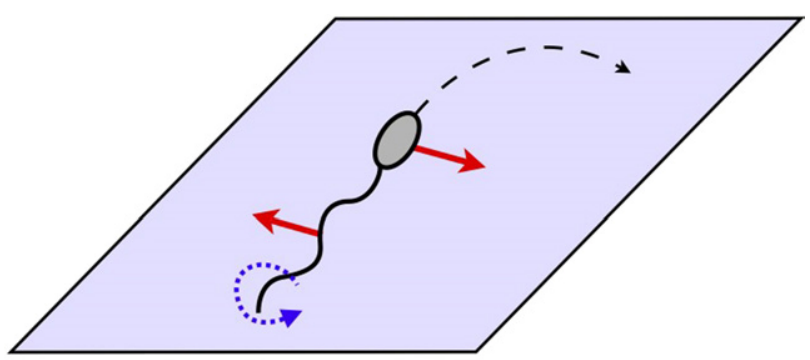

(b)

Figure 12. Bacteria swimming in circles near a boundary. (a) Circular trajectories for smooth-swimming E. coli bacteria near a glass surface. The motion of each bacterium is tracked for eight seconds (picture courtesy of Willow DiLuzio). (b) Physical mechanism for swimming in circles; the rotation of the bacterial flagella near the surface (blue dotted arrow) induces a net force on the flagella parallel to the surface but perpendicular to the flagella axis (red arrows, solid); an equal and opposite force is acting on the cell body due to its counter-rotation, resulting in a wall-induced torque acting on the cell, and a circular swimming trajectory (black arrow, dashed).

the helix in the direction perpendicular to the helix axis and parallel to the surface (figure 12(b), red solid arrow). In other words, when the helical flagella rotate, they create a net force on the cell at a right angle with respect to the motion and parallel to the surface. There is an exact and opposite force acting on the cell body, which rotates in the opposite direction as the flagella (figure $12(b)$, red solid arrows), and the net effect is a wall-induced torque. If the cell were to continue swimming in a straight line, it would have to apply a net torque on the surrounding fluid. Since a swimming bacterium is in fact torque-free, the cell cannot swim straight but instead rotates at a rate such that the viscous torque from that rotation exactly balances the wall-induced torque, and therefore swims along circles on the surface (figure 12(b), black dashed arrow). For a cell using a left-handed helix for propulsion, such as $E$. coli, this effect causes the cells to constantly turn to the right [224-229].

The third influence of boundaries on swimming cells is analogous to the attraction and reorientation induced by cellcell interactions and discussed in section 7.1. Consider a single cell moving near a solid wall. As the cell is swimming, it sets up a dipolar flow field, but this flow field in general does not satisfy the no-slip boundary condition on the wall, and images are necessary on the other side of the surface (see section 3.3). Because of the presence of images, a cell described by a dipole strength $p$, located at a distance $h$ from the surface, and pointing at an angle $\theta$ from the surface direction (figure 13(a)) is subject to the gradients of the image flow field, and as a result rotates with speed $\Omega \sim-p \theta / \eta h^{3}$ in the direction parallel to the surface and perpendicular to the cell body [204]. The $1 / h^{3}$ scaling originates from the leading-order vorticity of the image flow field, which is also dipolar. The rotation occurs as if the cell is interacting hydrodynamically with a mirror-image cell located on the other side of the surface, and the rotation rate is therefore analogous to that quantified by equation $(54)^{5}$. If the cell is a pusher, the wall-induced rotation rate, $\Omega=\mathrm{d} \theta / \mathrm{d} t$, tends to align the swimming cell in the direction parallel to the surface $(\theta=0$, figure 13(a)). As a

\footnotetext{
5 The effect of a flat boundary is mathematically equivalent to the presence of a mirror-image cell instantaneously located on the other side of the surface if the surface is a no-shear interface (e.g. a free surface). If instead it is a no-slip surface, the analogy is not quite exact mathematically, but it remains qualitatively correct.
}

(a)

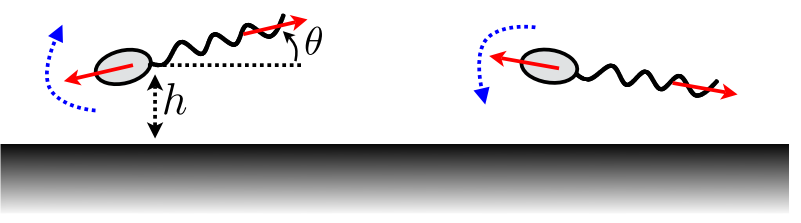

(b)

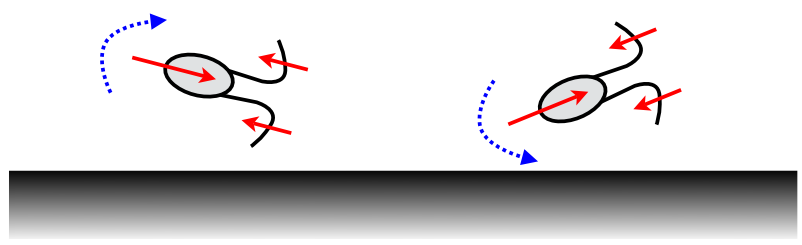

Figure 13. Wall-induced rotation of swimming cells. A swimming cell is located at a distance $h$ from a solid surface, and at an angle $\theta$ with respect to the direction of the surface. (a) Pushers are reoriented hydrodynamically in the direction parallel to the surface (equilibrium, $\theta=0)$; $(b)$ pullers are reoriented in the direction perpendicular to the surface (equilibrium, $\theta= \pm \pi / 2$ ).

result of this parallel configuration, the cells will swim in a sideby-side configuration with their image cell, and are therefore attracted to the surface with an attractive speed scaling as $u_{\perp} \sim$ $p / \eta h^{2}$ (see equation (53)). This physical picture explains the accumulation of swimming cells near surfaces observed in many biological experiments [198, 204, 223, 230-234]. Note that this wall-induced reorientation might be relevant to cellsorting and accumulation in funnel-like geometries [235]. Note that rotational diffusion also plays an important role [236]. In contrast, cells which are pushers are rotated in the opposite direction, and their stable configuration is instead at a right angle with respect to the surface $(\theta= \pm \pi / 2$, figure $13(b)$ ). In a confined environment, these cells are therefore always swimming toward one surface, a result which also leads to their accumulation. The physical origin is however different, and instead of being attracted by an image cell as pushers are, pullers simply swim into the wall.

The fourth hydrodynamic effect of boundaries, less studied, is a potential reduction of cell-cell hydrodynamic interactions near solid surfaces. Indeed, in many cases, a flow singularity at a distance $h$ from a solid surface is canceled out in the far field by its images on the other side of the surface. Thus, for distances greater then $h$, the overall flow decays faster in space than the original singularity. For example, a 


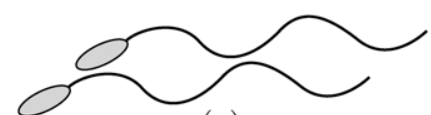

(a)

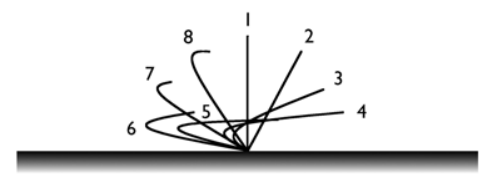

(c)

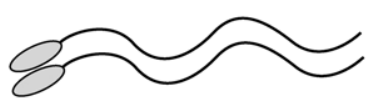

(b)

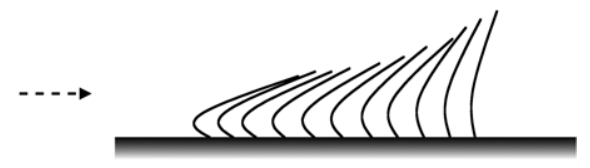

(d)

Figure 14. Hydrodynamic interactions between eukaryotic flagella and cilia. (a) Nearby swimming cells with arbitrary phase difference; $(b)$ phase locked nearby swimming cells; $(c)$ the asymmetric beat pattern of a cilium (sequence 1 to 10$) ;(d)$ schematic representation of the metachronal waves of collective beating displayed by arrays of cilia.

stokeslet near a wall has a dipolar behavior in the far field if the stokeslet is parallel to the surface, and a quadrupolar decay if it is perpendicular to it $[69,70]$. Swimming cells typically behave as force dipoles in an infinite fluid, but when near a solid surface, configurations exist where the total flow field (dipole + images) ends up decaying as $1 / r^{3}$ or $1 / r^{4}$, and hydrodynamic interactions with other cells are weaker as a result. In that case, the distance $h$ between the swimming cell and the wall acts therefore effectively as a cutoff for cell-cell interactions.

\subsection{Interactions between flagella}

7.3.1. Eukaryotic flagella and cilia. We now decrease our length scales, and discuss hydrodynamic interactions between cellular appendages. The first historical evidence of important hydrodynamic effects between nearby eukaryotic flagella were observations by James Gray of in-phase beating of spermatozoa flagella, later cited by Taylor [30]. Similar observations have been made since then, such as the synchronous beating of Fishfly spermatozoa [184]. Why would organisms phase lock in such a manner? Exploiting the swimming sheet model, Taylor showed that two swimmers with the same prescribed waveform (figure 14(a)) dissipate the least amount of mechanical energy for swimming when the waves are exactly in phase (figure $14(b)$ ), and the dissipation goes up monotonically with phase difference. Using highamplitude numerical simulations, these results were later revisited [237]. Two sheets with same waveform but different phases are seen to swim with different velocity, and their phase difference $\phi$ evolves until they are either perfectly in phase $(\phi=0)$ or perfectly out of phase $(\phi=\pi)$, with both configurations being stable [237]. Recent theoretical work showed that such phase-locking originates in the front-back asymmetry of the flagellar waveform of swimming cells [238].

Two nearby swimmers therefore display phase locking, but the locked state can be that of maximum dissipation, in contrast with what is observed experimentally. For real eukaryotes, the flagellar waveform is however not fixed but is found as a solution to a mechanical problem, as explained in section 6: the shape arises from a balance between internal forcing from molecular motors inside the flagellum, passive elastic or even viscoelastic resistance from the axoneme, and viscous resistance from the outside fluid. When there is a second swimmer located nearby, the fluid forces are modified, changing the balance of force and thus the flagellar shape. Through this neighbor-induced change in the waveform, cells are expected to be able to perfectly phase lock, as is observed in the synchronization of nonlinear oscillators [239].

Most of the work related to hydrodynamics-induced waveform change and phase locking was concerned with cilia, which are short eukaryotic flagella (a few micronmeters long), found in densely packed arrays and involved in many important biological functions, including sensing, fluid transport, locomotion, and development [3, 13,21, 59, 240-242]. An individual cilium deforms in a non-reciprocal fashion, with a typical high-friction power strokes (sequence 1 to 3 in figure $14(c)$ ) and a low friction recovery stroke (sequence 4 to 8 in figure $14(c)$ ). The internal actuation of each cilium is independent from that of its neighbors, and they do not communicate with each other except through the fluid. However, when they are closely packed on surfaces, cilia arrays display collective behavior, termed metachronal waves. The deformation of each cilium is locked in phase with that of its neighbor, with a small constant phase difference, leading to propagating waves of deformation (figure 14(d)) [59].

The physical origin of coordinated beating is the central question of ciliary dynamics, which a number of theoretical studies have attempted to answer [117-120,243-246]. Two different approaches have been proposed. In the first one, the mechanics of each cilium is modeled as accurately as possible, and numerical simulations are used to compute the collective beating [117-120]. The crucial ingredient in that approach is to correctly model the internal load-dependent force generation in the axoneme (without load dependence, or feedback, there is no phase locking as can be expected [246]). With that approach, it is found that two cilia starting randomly end up beating in perfect synchrony within two beating cycles [119]. If instead there are a large number of cilia, waves arise naturally as a result of hydrodynamic interactions [119]. Subsequent work showed that as the waves develop from arbitrary initial conditions the rate of work done by the cilia as they are beating is decreasing [120]. Physically, because of viscous drag, it is energetically advantageous for one cilium to beat in the presence of a neighboring cilium with a similar phase.

The second approach considers simplified models for the dynamics of the cilia, providing analytical insight into the 

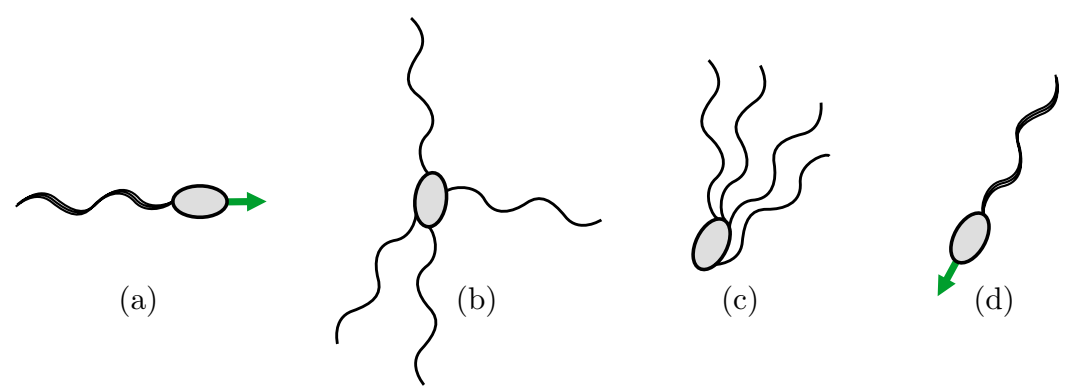

Figure 15. Bundling of bacterial flagella. During swimming, the bacterial flagella are gathered in a tight bundle behind the cell as it moves through the fluid $((a)$ and $(d))$. During a tumbling event, the flagella come out the bundle $(b)$, resulting in a random reorientation of the cell before the next swimming event. At the conclusion of the tumbling event, hydrodynamic interactions lead to the relative attraction of the flagella $(c)$, and their synchronization to form a perfect bundle $(d)$.

necessary conditions for phase locking [243-246]. An early study considered a regular lattice of cilia, where the direction of the beating plane is assumed to obey a balance between rotational Brownian motion and rotation induced by the flow created by all other cilia. For small enough temperature, a transition is observed between a state with no average net flow, and a state where all cilia point in the same direction and drive a net flow [245]. Further modeling is provided by considering a simplified load-dependent internal molecular engine. In that case, metachronal waves arise only if a constant phase shift is assumed to exist between each cilium and its neighbor [245].

Motivated by nodal flows in development [242], a second study considered cilia whose tips perform three-dimensional trajectories over a surface. Each cilium is modeled by a sphere subject to an active load-dependent force, and interacting hydrodynamically with a second cilium. Depending on the relative position and orientation of the two cilia models, inphase $(\phi=0)$ or out-of-phase locking $(\phi=\pi)$ arise from random initial conditions [244]. A similar model with two sphere-like cilia rotating due to an applied torque near a wall was recently proposed. In that case, in-phase locking is obtained provided that the circular trajectory of each cilium is allowed to vary in response to hydrodynamic interactions [246].

A recent table-top experiment has been used to examine the physics of hydrodynamic synchronization [247]. In this work, a pair of centimeter-sized rectangular paddles are immersed in silicone oil with a viscosity 100000 times that of water. The paddles are rotated by motors that deliver constant torque. In accord with the theoretical results of [246], a small compliance was required for phase locking. The time scale for synchronization was long compared with the paddle rotation period, and is governed by the strength of the hydrodynamic interaction between the paddles. To achieve steady phase locking, the driving torques had to be closely matched.

\subsubsection{Bacterial flagella. Hydrodynamic interactions} between flagella also play a pivotal role for bacterial locomotion. In that case, the phenomenon of interest is flagellar bundling [248, 249]. Wild-type swimming bacteria, such as E. coli, typically display 'run-and-tumble' behavior during their locomotion. During runs, the bacterium swims along a roughly straight path, and its flagellar filaments are bundled together tightly behind the cell (figure 15(a) and $(d)$ ). Near the end of a run, one or more motors reverses, and the corresponding filaments unwind from the bundle (figure 15(b)). Viscous stresses lead to polymorphic transitions, in which a flagellum changes from a left-handed to a right-handed helix. There are also polymorphic transitions between different right-handed helices. These transitions are correlated with changes in swimming direction. Once the motors reverse again, the full bundle forms (figure 15(c), then $(d)$ ), and the cell heads off in a new direction [23] (figure $15(d)$ ). Since the flagella, which are randomly distributed along the cell's surface, do not communicate except through the fluid, it has long been postulated that bundling occurs because of hydrodynamic interactions.

During bundling, two different physical mechanisms are involved: (1) the attraction between the rotating flagella and (2) the phase locking of nearby flagella. Concerning the attraction between the rotating flagella, two different scenarios have been proposed, and both play a role. One is purely kinematic, and relies on the simple observation that as the cell starts swimming, the drag on all the flagella naturally sweeps them behind the cell [248]. Under this scenario, the flagella are not so much attracted by each other, but are simply passively dragged behind the cell body. The wrapping of the filaments is then achieved in a passive manner by the body rotation arising from overall torque balance (see section 5.2) [248, 250]. The second attraction scenario relies on hydrodynamic interactions between the flagella, in which each helix induces a flow which causes the other to bend and twist about its neighbors. Even without the counter-rotation of the body, the flows induced by rotating flexible helices can cause bundling [251]. The geometry of the helices is critical for this scenario. The role of this geometry has been studied with macro-scale experiments using flexible helices in air [249]. In these experiments, the bundling was forced by means of guides at the distal ends of the helices. It was found that left-handed helices rotating counter-clockwise as viewed from the distal end (the same handedness and rotation sense as a bacterium on a run) can maintain a steadily rotating bundle, without jams, when the helices are wrapped around each other in a right-handed sense. Later, macro-scale experiments using polymeric helices in highly viscous silicon oil were developed to study how hydrodynamic interactions could lead to bundling [251,252]. There it was shown that induced flow from rotating helices 
naturally causes tight bundling of pairs of left-handed helices driven counter-clockwise. The only other combination that led to bundling was its mirror image, with right-handed helices driven clockwise [251]. The induced flows cause the helices to bend and cross each other with the centerlines of each helix slightly curved along helical paths. Once the helices touch, they quickly wrap up to form a tight bundle, which is maintained indefinitely as long as the rotation speed is not too high. The time for bundling in the experiment was observed to scale with the rotation speed of the helix, rather than the relaxation time of flexible helices in a viscous fluid. Bundling has also been studied computationally using a regularized version of slender-body theory [253]. For three left-handed helical flagella driven at constant torque and rotating counterclockwise, hydrodynamic interactions lead to a bundling of the filaments, although not as tight a bundle as observed experimentally [253].

The issue of synchronization between helices driven with constant torques was addressed numerically [254, 255]. Perhaps surprisingly, two nearby rotating helices are seen to not synchronize if the helices are perfectly rigid [254], and some mechanical flexibility is necessary for flow-based synchronization to occur [255]. In bacteria, this flexibility is likely to be provided by the flagellar hook which connects the rotary motor to the helical flagellum [256,257]. The contact forces that arise between different helices as the bundle forms likely also play a role in the phase-locking of flagella in a bundle.

\section{Swimming in complex fluids}

Biological fluids are often laden with polymers and therefore have non-Newtonian rheology. For example, mucus is found at the cervix and throughout the rest of the mammalian female reproductive tract [2]. The nature of the cervical mucus influences the likelihood of fertilization in humans: there is a good correlation between the hydration of the cervical mucus and the incidence of pregnancy [258]. Although cervical mucus is a complex, heterogeneous gel, some simple trends have been observed. There is an inverse relation between the degree of viscoelasticity of the mucus and the ability of sperm to penetrate the mucus [259-262]. Human sperm flagella in cervical mucus have a higher beat frequency, smaller amplitude, and shorter wavelength than when in semen [263]. The swimming speed is the same in both media, but the sperm swim along straighter paths in cervical mucus [263]. In addition to the mucus at the cervix and in the uterine cavity, sperm must also penetrate the matrix coating the ovum, known as the cumulus oophorus, which is a viscoelastic actin-based gel [264]. Spermatozoa can undergo an internal change known as hyperactivation in which the beat pattern changes from a symmetric to asymmetric form; hyperactivated cells have better motility in viscoelastic media [265-269].

Rheological measurements show that cervical mucus is highly viscous, with a viscosity of $\approx 100 \mathrm{Pas}$ [270]. The viscoelasticity is characterized by a relaxation time of $\lambda \approx 1-10$ s $[261,271-273]$ and an elastic modulus of $\approx 0.1-$ $10 \mathrm{~Pa}$ s $[261,273]$. These properties depend strongly on the phase of the menstrual cycle as well as hydration. Since the typical beat frequency of a flagellum is $\omega /(2 \pi) \approx 20$ $50 \mathrm{~Hz}$ [13], the Deborah number $\omega \lambda$ is easily larger than one, indicating that viscoelastic effects are important.

There are several other important examples of swimming or transport in complex fluids. The cilia that line the human upper airway lie in a thin layer of Newtonian liquid, which in turn is coated with a high-viscosity layer of mucus [59]. Again, since the Deborah number for this system is large [274], elastic effects are important. Bacteria also encounter viscoelastic fluids: the ulcer-causing bacterium Helicobacter pylori swims through mucus lining the stomach [275], and spirochetes move through the connective tissue of the host during infection [276].

In our discussion of swimming in a complex fluid, we use continuum mechanics to model the fluid. This simplification is necessary for making progress, but it is important to realize that the size scale of the microstructure of the fluid can be comparable to the size of the swimmer, and therefore a different approach may be necessary to accurately capture the interactions between the swimmer and the polymers. Complex fluids display a vast array of non-Newtonian effects, such as stress relaxation, normal stress differences and shear-rate dependent viscosity [206-213]. Our approach is to illustrate some of the distinctive properties of swimmers in complex fluids by focusing on one class of models, fluids with fading memory. These models apply to polymer solutions.

When a polymer solution is subject to shear, the polymers stretch out. The resulting loss of entropy of the polymers leads to an effective elastic force tending to recover the initial configuration of the polymers. The balance of the entropic force and the viscous drag on the polymer sets the time scale $\lambda$ over which the fluid has memory. The appearance of this new time scale gives polymeric liquids a completely different character relative to viscous Newtonian fluids. First, the property of kinematic reversibility is lost, even if the Reynolds number is vanishingly small, since growth or decay of stress can lag the change in shape of a swimmer by the time scale $\lambda$. Second, the new time scale implies that the constitutive relation for a polymer solution depends on the rate of change in stress with time, which automatically leads to nonlinear terms. This fact may be seen by invoking material frame indifference, a fundamental assumption of continuum mechanics [205, 207], or by deriving the continuum theory directly from a microscopic theory of the polymers in solution [211,213]. Many of the properties of Stokes flow that we have invoked in our study of swimming have explicitly relied on the linearity of the equations of motion. The nonlinearity of the equations of motion for a polymeric liquid generally implies that the scallop theorem does not hold. The loss of linearity spoils superposition, and therefore we may not use the singular solutions of section 3.3 to construct a slenderbody theory. Hydrodynamic interactions between distant cells will have a different character than in Stokes flow, since the far field form of the velocity field due is changed. These features of polymeric liquids make the study of swimming in complex fluids a daunting challenge, but also an area of opportunity. Except for a few early works [277-279], the area is largely unexplored. 
To examine these issues, we review the calculation [274] of the swimming velocity of the Taylor sheet of section 5.1 for a fluid described by the Oldroyd-B model [207]. In this model, the deviatoric stress $\tau=p \mathbf{1}+\boldsymbol{\sigma}$ is the sum of a contribution from the polymer solute $\tau^{\mathrm{p}}$ and a contribution from the Newtonian solvent $\boldsymbol{\tau}^{\mathrm{s}}=2 \eta_{\mathrm{s}} \boldsymbol{E}$, where $\boldsymbol{E}=\frac{1}{2}\left(\nabla \boldsymbol{u}+\nabla \boldsymbol{u}^{\mathrm{T}}\right)$. The polymer contribution relaxes to the viscous stress over the time scale $\lambda_{1}$,

$$
\tau^{\mathrm{p}}+\lambda_{1} \stackrel{\nabla}{\tau^{\mathrm{p}}}=2 \eta_{\mathrm{p}} \boldsymbol{E},
$$

where $\eta_{\mathrm{p}}$ is the polymer contribution to the viscosity, and

$$
\stackrel{\nabla}{\boldsymbol{\tau}}=\frac{\partial \boldsymbol{\tau}}{\partial t}+\boldsymbol{u} \cdot \nabla \boldsymbol{\tau}-\boldsymbol{\tau} \cdot \nabla \boldsymbol{u}-(\nabla \boldsymbol{u})^{\mathrm{T}} \cdot \boldsymbol{\tau}
$$

is the upper-convected derivative of $\tau$. The upper-convected derivative of a tensor $\tau$ is the expression in general coordinates of the rate of change of the tensor calculated in a frame that translates and deforms with the local fluid velocity [205, 207]. Using $\tau=\tau^{\mathrm{p}}+\tau^{\mathrm{s}}$ and eliminating $\tau^{\mathrm{p}}$ from equation (57) yields the Oldroyd-B model,

$$
\tau+\lambda_{1} \stackrel{\nabla}{\tau}=2 \eta\left(\boldsymbol{E}+\lambda_{2} \stackrel{\nabla}{\boldsymbol{E}}\right),
$$

where $\eta=\eta_{\mathrm{p}}+\eta_{\mathrm{s}}$ and $\lambda_{2}=\eta_{\mathrm{s}} \lambda_{1} / \eta<\lambda_{1}$. The equation of motion is $-\nabla p+\nabla \cdot \boldsymbol{\tau}=0$ with $\nabla \cdot \boldsymbol{u}=0$. Note that the explicit presence of the time derivatives as well as the nonlinear terms in equation (59) spoils kinematic reversibility and violates the assumptions of the scallop theorem.

The solution to the swimming problem for a sheet with prescribed traveling wave (20) proceeds just as in the Newtonian case. The velocity field, stress and boundary conditions are expanded to second order in amplitude $b$, and the governing equations are solved subject to the boundary conditions (21) and (22). To first order, the flow field is identical to the flow field of the Stokes problem. However, due to the relaxation time $\lambda$, the first-order component of the stress field $\tau$ has a lag relative to the Stokes stress field. Through the nonlinear terms of equation (59), this stress field drives a second-order flow that leads to the swimming speed [274]

$$
|\boldsymbol{U}|=\frac{1}{2} \omega k b^{2} \frac{1+\omega^{2} \lambda_{1}^{2} \eta_{\mathrm{s}} / \eta}{1+\omega^{2} \lambda_{1}^{2}} .
$$

Since $\eta_{\mathrm{s}}<\eta$, the swimmer in an Oldroyd-B fluid is slower than in a Stokes fluid. Note that unlike the Stokes case, the swimming speed (60) depends on material parameters such as $\lambda_{1}, \eta_{\mathrm{s}}$ and $\eta$. The Oldroyd-B constitutive equation is inapplicable to flows with large extension rates. Nevertheless, the result (60) continues to hold for more accurate fading memory models such as FENE-P, the Johson-SegalmanOldroyd model and the Giesekus model [274]. The expression for the speed, equation (60), also continues to hold for a cylindrical filament with a traveling wave, in the limit in which the radius of the cylinder is much smaller than the lateral displacement of the cylinder [160,280]. Further work extended these small-amplitude calculations to the case of three-dimensional finite-size bodies and derived an integral theorem quantifying the swimming kinematics for locomotion in an arbitrary complex fluid [281].
As a result of the presence of nonlinear evolution equations, locomotion in complex fluids escapes the constraints of the scallop theorem, from two different distinct origins. The first breakdown occurs through rate-dependence, as illustrated by the nonlinear dependence of the swimming speed on the frequency in equation (60). To see why the theorem is no longer valid, first consider the Newtonian limit $\lambda_{1} \rightarrow 0$, in which all elastic effects vanish and the speed is proportional to $\omega$. Now consider a situation in which a traveling wave of wave number $k$ and frequency $\omega$ travels rightward on the sheet for a period $(2 \pi / \omega) / 3$, and then leftward with frequency $\omega / 2$ and period $2(2 \pi / \omega) / 3$ [280]. The deformation of the sheet is reciprocal, with a rightward wave of speed $c_{1}=\omega / k$, followed by a leftward wave of speed $c_{2}=c_{1} / 2$. From the scallop theorem, the sheet will have zero net displacement after this process. Now consider this waveform for a sheet in an Oldroyd-B fluid. Suppose the sheet has periodically been executing these motions long enough that transients from startup from rest have died away. Since the speed now depends nonlinearly on $\omega$, the translation in each segment of the motion is different: the sheet moves faster during the leftward motion with the smaller frequency. Thus, there is a net displacement. This argument disregards the memory of the fluid, since the stress during each stroke is effected by the previous stroke. Accounting for these memory effects leads to a slightly smaller net displacement, but it still has the same qualitative dependence on $\omega \lambda_{1}$.

The second breakdown of the scallop theorem occurs through the presence of nonlinear rheological properties. In that case, even in the limit of a periodic motion occurring at the same rate back and forth, the stretching of the polymeric molecules in the fluid leads an additional tension along the flow streamlines, resulting in additional normal stresses scaling quadratically with the imposed shear [207]. Such nonlinear rheological properties mean that net forces and flow can be created out of a purely sinusoidal motion [282]. For example, the small-amplitude periodic flapping of a semi-infinite plane in an Oldroyd-B fluid leads to the development of net timeaveraged stresses, $\langle\delta \boldsymbol{f}\rangle$, acting along the flapper and scaling as

$$
\langle\delta \boldsymbol{f}\rangle \sim \epsilon^{2} \frac{\lambda_{1} \omega}{1+\left(\lambda_{1} \omega\right)^{2}}\left(1-\frac{\eta_{\mathrm{s}}}{\eta}\right) \eta \omega,
$$

where $\epsilon$ is the amplitude of the flapping angle [282]. Similarly, a spherical swimmer of radius $R$ subject to a small-amplitude tangential motion varying sinusoidally in time moves with a time average speed, $\langle\boldsymbol{U}\rangle$, scaling as

$$
\langle\boldsymbol{U}\rangle \sim \epsilon^{2} \frac{\lambda_{1} \omega}{1+\left(\lambda_{1} \omega\right)^{2}}\left(1-\frac{\eta_{\mathrm{s}}}{\eta}\right) R \omega,
$$

where $\epsilon$ is the relative amplitude of the tangential motion [281]. For both cases, in the Newtonian limit we have $\lambda_{1}=0$ and the scallop theorem is recovered $(\langle\delta \boldsymbol{f}\rangle=\langle\boldsymbol{U}\rangle=\mathbf{0})$ but for all other cases the time-reversible motion induces non-zero time-averaged forces and speeds.

The properties of the medium can affect not only the speed of a swimmer with a prescribed stroke, but also the shape of a beating flagella. For example, as the viscosity of the solution 


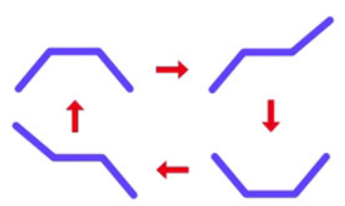

(a)

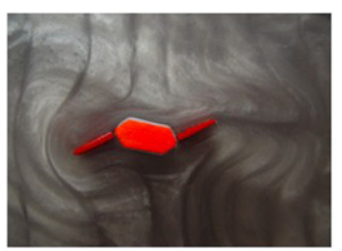

(b)

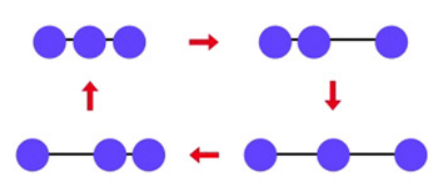

(c)

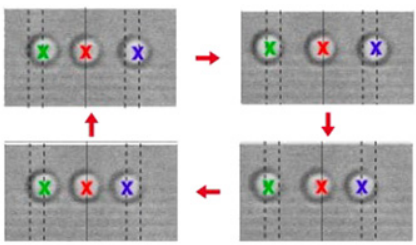

(d)

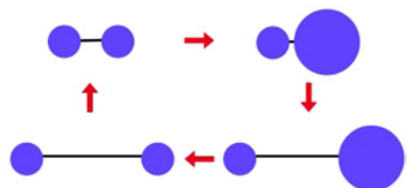

(e)

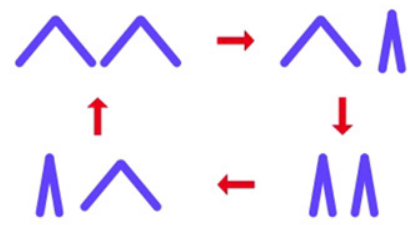

(f)

Figure 16. Artificial swimmers with discrete degrees of freedom: $(a)$ Purcell's three-link swimmer (red arrows indicate change in conformation of the swimmer shape); $(b)$ macro-scale experimental realization of Purcell's swimmer (photo courtesy of Brian Chan and Peko Hosoi); $(c)$ three-sphere swimmer; $(d)$ micro-scale realization of a three-sphere pump [285]; $(e)$ two-sphere swimmer with shape change; $(f)$ two scallops together can make a non-reciprocal sequence of shapes, leading to net displacement. Picture in figure $16(d)$ adapted from [285] courtesy of Marco Cosentino Lagomarsino, and reproduced by permission of The Royal Society of Chemistry, copyright 2009 (doi:10.1039/b812393d).

increases, the wave form of a human sperm flagellum flattens along most of its length, with most of the deflection taking place at the distal end [283]. Thus it is natural to model the dependence of the shape on material parameters such as viscosity and relaxation time. As we learned in section 6 , the small-amplitude shape is determined by an equation which is first order in amplitude. Thus, we only need the fluid force to first order. For the Oldroyd-B fluid, the calculations for the filament geometry show that this fluid force has the form of resistive force theory, with complex frequency-dependent effective viscosity [94]. Denoting Fourier components with a tilde, the shape equation for an Oldroyd-B fluid is

$$
\frac{\mathrm{i} \omega \xi_{\perp}\left(1+\mathrm{i} \lambda_{2} \omega\right)}{1+\mathrm{i} \lambda_{1} \omega} \tilde{h}=-A \frac{\partial^{4} \tilde{h}}{\partial x^{4}}-a \frac{\partial \tilde{f}}{\partial x},
$$

where $f$ is the sliding force density. By prescribing the force density, imposing the boundary conditions and using linear superposition (valid to first order), we may calculate the shape of the filament as a function of material parameters. The result is that this model gives patterns that look qualitatively similar to the experimental observations [94]. Since the shape of the filament determines the swimming velocity, the dependence of shape on relaxation time gives an additional correction to the swimming velocity of the prescribed sheet.

A natural next step is to extend the study of swimming to large-amplitude deflections. Recent numerical work on largeamplitude peristaltic pumping of an Oldroyd-B fluid in two dimensions has shown similar qualitative differences with the Newtonian case, such as reduced pump rate, dependence on material parameters and loss of kinematic reversibility [284]. The application of numerical methods such as these to swimmers will be crucial for understanding finite-size effects such as the role of the shape of the cell body or the end of the flagellum, since large normal stresses can develop at such regions of high curvature.

\section{Artificial swimmers and optimization}

In this final section, we look beyond the biological realm and survey the bioengineering advances in design and optimization of artificial swimmers and bio-inspired systems.

\subsection{Designing artificial swimmers}

A number of designs have been proposed and tested for artificial swimming devices at low Reynolds number. They can be sorted in three different categories. The first category includes all swimmers which deform their shape with only a finite number of degrees of freedom, actuated in a nonreciprocal fashion. At least two degrees of freedom are needed for swimmers in this category. The original example is Purcell's three-link swimmer, which posseses two hinges varying in time with phase differences (figure 16(a) and $(b)$ ) $[14,78,73,286]$. A second related design is that of a threesphere swimmer (figure 16(c)): the distances separating the spheres vary in time and with phase differences, leading to locomotion [82,187,287-290]. The extension to $\mathrm{N}$ spheres was also proposed theoretically [291]. The three-sphere design was implemented experimentally using optical tweezers, and the out-of-phase motion of the three colloidal beads leads to fluid pumping (figure 16(d)) [285]. A third design only needs two spheres, but is coupled with a change in shape of one of the spheres, leading to non-reciprocal kinematics (figure 16(e)). As the sphere increases in size, it serves as an anchor against which the swimmer can push, and rectify a time-periodic change in size [292]. A related idea using rotating two-sphere swimmers, was also proposed [293]. Finally, if the phases of nearby bodies individually undergoing reciprocal motion are suitably adjusted so that together the sequence of shapes is nonreciprocal [294], then hydrodynamic interactions between the body can lead to net propulsion (figure $16(f)$ ). The resulting collective swimming speeds depend on the separation distance between the swimmers as well as their relative position and orientation [80, 295]. 


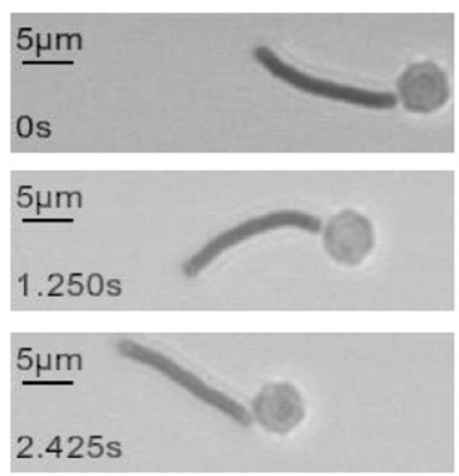

(a)
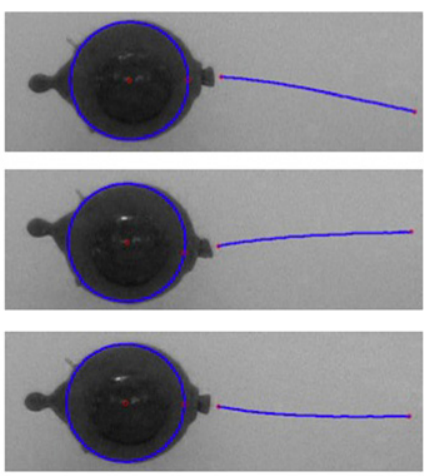

(b)

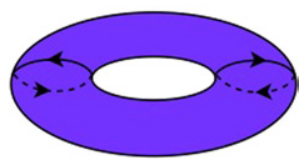

(c)

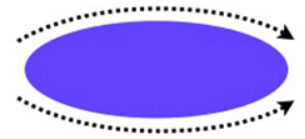

(d)

Figure 17. Artificial swimmers with continuous deformation: (a) synthetic flagella made of actuated micrometer-scale magnetic filaments [296]; (b) macro-scale swimming by boundary actuation of an elastic filament [297]; (c) toroidal swimming; $(d)$ swimming by surface treadmilling. Picture in figure 17( $a$ ) adapted from [296] courtesy of Remy Dreyfus and by permission from Macmillan Publishers Ltd, copyright 2005 (doi:10.1038/nature04090).

The second category of artificial swimmers consists of bodies deforming in a continuous fashion. In an experimental breakthrough, a team has been able to exploit the response of paramagnetic filaments to external magnetic fields to construct the first artificial micrometer-long swimmer (figure 17(a)) [296], thereby motivating a number of theoretical studies [298-302]. In this work, an ac magnetic field is applied in the direction perpendicular to the filaments, and the presence of a body (here, a red blood cell) breaks the right-left symmetry in the continuous response of the filament. The result is a wave of deformation traveling from the tip of the filament to the body it is attached to, and locomotion flagella-first [296]. A second type of swimmer with continuous deformation exploits the boundary actuation of elastic filaments to generate propulsive forces (see section 6) and locomotion (figure 17(b)). In a macro-scale experiment, the forces arising from such elastic swimming were measured [143], and the actuation method was implemented successfully to obtain swimming [144,297]. A third type of swimmers with continuous deformation are those with a special mode of surface deformation called 'surface treadmilling', for which the shape of the swimmer is fixed and where its body undergoes a continuous tangential displacement along its surface (figures $17(c),(d)$ ). Taylor proposed such design in the shape of a torus undergoing continuous surface rotation (figure 17(c)) [95], an idea recently analyzed in detail [303-305]. If the body is slender and displays directed tangential displacement all along its surface, so that a material source is present on one side of the body, and a sink on the other side (figure $17(d)$ ), the resulting locomotion can occur with arbitrarily high efficiency [306].

The final category of artificial swimmers uses chemical reactions to power locomotion, a case investigated both experimentally [285, 307-314] and theoretically [315-318]. The prototypical setup is a body composed of two materials, one that is inert and one that is a catalyst or reactant for a chemical reaction. The presence of a chemical reaction leads to a concentration gradient for some of the reactants and/or products of the reaction, leading to slip velocities at the particle surface and relative motion between the particle and the fluid $[319,318]$. An example of the trajectory of such chemical swimmer is illustrated in figure 18 .

\subsection{Exploiting low-Re locomotion}

The physical mechanisms of propulsion at low Reynolds number can be exploited in a number of ways beyond biological or synthetic locomotion. In a pioneering experiment, suspensions of swimming bacteria were seen to provide an effective high-temperature thermal bath for suspended inert particles [176]. Similar results were obtained for diffusive mixing of two fluids containing swimming cells [320], and for the motion of colloidal particles above surfaces covered with attached bacteria (figure 19) [321]. A second utilization of swimming is cargo-towing, with potential applications in biomedical devices. Experiments were performed with solid bodies covered with attached bacteria, with successful translational and rotational towing [321]. A related method was also devised to transport micrometer-size objects at the single-cell level [322], and a theoretical framework for towing by swimming now exists [323]. A final application of flagella motion is pumping. Indeed, swimming and pumping are dual problems, and in general a tethered swimmer acts as a pump [324]. One approach, studied numerically, uses elastic filaments attached to a solid surface and actuated by external time-varying torques [325]. Experiments were also conducted on surfaces covered with filaments made of self-oscillating gels and displaying wave-like motion [326].

\subsection{Optimization}

Motivated by the optimal tuning of synthetic micro-swimmers, as well as possible insight into evolutionary processes, significant work has been devoted to the optimization of locomotion at low Reynolds number. Since time can be scaled out of all low Reynolds number swimming problems in Newtonian fluids, optimizing swimming speeds is not, in general, a well-posed mathematical problem. For example, if a given swimmer is made to deform its surface twice as fast, it will swim twice as much per unit time, and hence would be seen to be a better swimmer, yet the geometry of its swimming gait would not have changed. To render optimal swimming a well-posed mathematical problem, a form of normalization is required. The first approach consists of optimizing the 


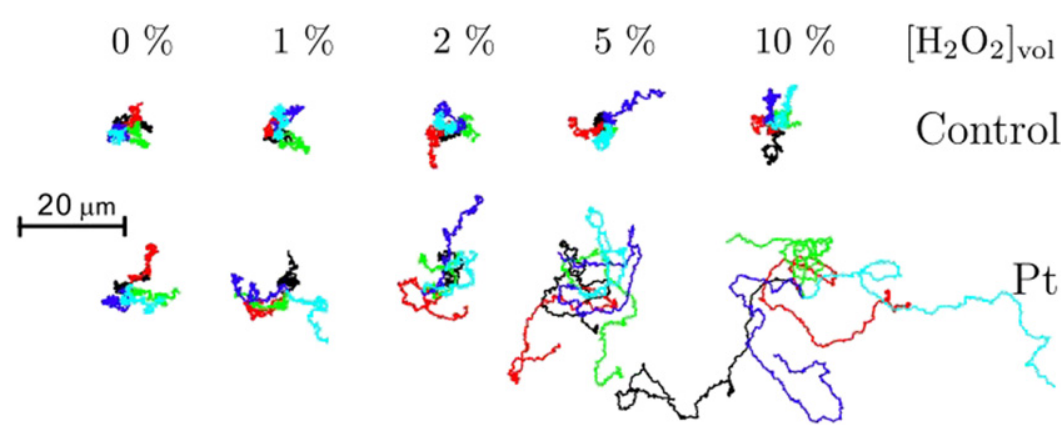

Figure 18. Locomotion powered by chemical reactions [313]. Polystyrene colloidal spheres in a solution of water and hydrogen peroxide $\left(\left[\mathrm{H}_{2} \mathrm{O}_{2}\right]\right)$ with increasing concentration. Top: control experiment (inert spheres). Bottom: experiment where the spheres are half-coated by platinum, a catalyst for the reduction of $\left[\mathrm{H}_{2} \mathrm{O}_{2}\right]$ into oxygen and water, which leads to directed swimming of the spheres (coupled to Brownian rotational diffusion). Picture reproduced from [313] courtesy of Ramin Golestanian and by permission from the American Physical Society, copyright 2007 (doi:10.1103/PhysRevLett.99.048102).
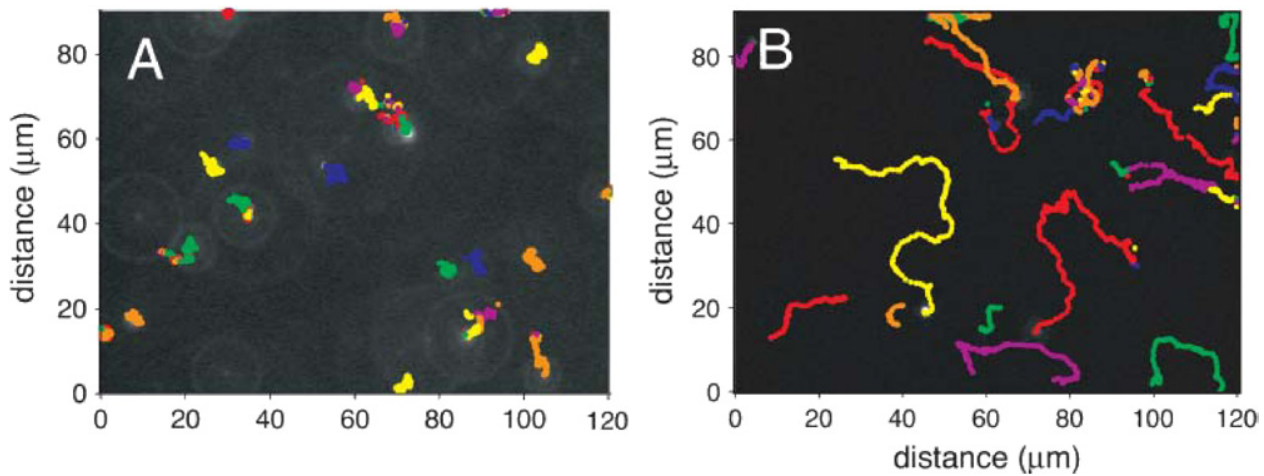

Figure 19. Enhanced diffusion near a carpet of swimming cells [321]. Trajectory of fluorescent particles over a $10 \mathrm{~s}$ interval when located above a flat surface coated with attached bacteria (two-dimensional view from above the surface). (a) The particles are located $80 \mu \mathrm{m}$ above the surface, and move by Brownian motion. $(b)$ When the particles are located $3 \mu \mathrm{m}$ above the surface, their effective diffusion is significantly larger than Brownian motion [321]. Picture reproduced from [321] courtesy of Kenny Breuer and by permission from the Biophysical Society, copyright 2004 (doi:10.1016/S0006-3495(04)74253-8).

distance traveled by the swimmer per unit period of its timeperiodic body deformation [286], thereby explicitly making the optimality question a geometrical problem. A second method, more traditionally used, consists of defining a swimming efficiency as the ratio of the useful to the total rate of working of the swimmer against the viscous fluid (see section 5.2). The optimization problem becomes a maximization problem for the efficiency [11], and is equivalent to finding the swimmer with the largest swimming speed for a given amount of mechanical energy available. Since both rates of working are quadratic in the rate of deformation of the swimmer, this method also effectively solves the normalization issue. In the paragraphs below we use the term 'optimization' to refer to the problem of swimming with maximum efficiency.

Two types of optimization questions can be asked, related to either the waveform or the overall geometry of the swimmer. The first problem was formulated by Lighthill as the following: if a flagellum is being distorted as a planar traveling wave, what would be its optimal waveform [11]? Assuming an infinitely long flagellum, and within the framework of the local drag theory, the optimized flagellum is a sawtooth wave, where each branch of the sawtooth makes an angle $\pm \theta$ with the horizontal, with $\theta=\arccos \left[\left(1+\left(\xi_{\|} / \xi_{\perp}\right)^{1 / 2}\right)^{-1 / 2}\right]$ [11]. Since $\xi_{\|} / \xi_{\perp} \approx 1 / 2$, the optimal angle for the sawtooth is $\theta \approx 40^{\circ}$, leading to an optimal value for the product of the wave amplitude, $b$, and the wavenumber, $k$, as $b k \approx$ 1.3. The resulting optimal hydrodynamic efficiency is about $8 \%$ [11], significantly above the efficiency of about $1 \%$ typically displayed by biological cells $[3,11,28,105]$. Note that the optimal value for $\theta$ is insensitive to the exact value of the ratio $\xi_{\|} / \xi_{\perp}$, or on the possible presence of a cell body [11]. Numerical simulations for sawtooth patterns revealed the optimal criterion of Lighthill to remain valid for finite-size swimmers [327]. In addition, flagella with the optimal sinusoidal deformation have very similar geometrical characteristics: $b k \approx 1.26$ using the local drag theory [328], and $b k \approx 1$ using slender-body theory [329]. Similar results are obtained by numerical optimization of the flagellar waveform [105].

The existence of an optimal angle between the direction of swimming and the direction of the flagellum can be understood on the basis of the physical picture we introduced for dragbased propulsion (figure 2). Indeed, we see from equation (13) that the propulsive force arising from the local motion of the flagellum scales with its orientation as $f_{\text {prop }} \sim \sin \theta \cos \theta$. If the work done by the flagellum were not a function of its orientation, the optimal angle would therefore be the one for which $\sin 2 \theta \sim 1$, or $\theta \sim 45^{\circ}$. Because $\xi_{\perp}>\xi_{\|}$, some energy 
can be saved by decreasing the angle, thereby promoting tangential over perpendicular motion, which explains why the optimal angle is close to, but below, $45^{\circ}$. In the case of planar waves, the optimal flagellum waveform is therefore kinked like a sawtooth pattern, and the slope angle alternates between $+\theta$ and $-\theta$. In the case of a three-dimensional helical filament, a constant angle between the local tangent along the flagellum and the swimming direction can be accommodated while keeping a smooth waveform if (and only if) the flagellum takes the shape of a helix. In that case, the optimal angle was determined numerically using slender-body theory to be $\theta=45^{\circ}$ [330] (see also [65, 331]).

A second design feature which can be optimized is the overall geometry of the swimming cell, in particular the ratio of the length $L$ of its flagellum and the radius $a$ of its body, which is assumed to be spherical for simplicity. In the case of swimmers exploiting planar waves, this problem can only be studied if hydrodynamic interactions between the body and the flagellum are properly taken into account, as otherwise the presence of a body always decreases the swimming efficiency (see equation (38)). This problem was analyzed in detail using slender-body theory for sinusoidal waveforms, and the optimal flagellum length to body size ratio was found to be $L / a \approx 25$ [329]. Subsequent work determining the optimal flagellar waveforms showed that the optimal ratio is close to $L / a \approx 24$ [105]. In the case of helical waves, an optimal body size can be determined even in the absence of hydrodynamic interactions. Indeed, we saw in section 5.2 that cells with helical flagella need a body in order to be able to move. A small body prevents the swimmer from moving and is not efficient, whereas a large body presents too large a drag to the overall cell, and is not efficient either: an optimal size must therefore exist. When hydrodynamic interactions are also taken into account, the optimal ratio has been determined numerically to be $L / a \approx 10$ using slender-body theory [330] and $\approx 12$ using three-dimensional boundary elements [332] (see also [65]). Other biologically relevant optimization problems that have been addressed include the optimization of feeding current for tethered cells [333], and the influence of the body shape on the optimal flagellar waveform [105] and overall cell geometry [332].

Finally, work has been devoted to the optimization of simple artificial swimmers. For bodies with discrete degrees of freedom, the actuation of Purcell's swimmer (figure 16(a)) was optimized numerically [78, 286]. More formal mathematical work was devoted to the optimization of the three-sphere swimmer (figure 16(c)) using a controltheory framework [334] and axisymmetric swimmers in general [335]. For swimmers with continuous degrees of freedom, the geometrical approach to swimming at low Reynolds number was used to derive optimal lowamplitude swimming by surface deformation of spheres and cylinders [74-76], and was later extended to large-amplitude deformation of two-dimensional bodies [336]. In the case of a spherical swimmer with time-periodic tangential deformation, the hydrodynamic efficiency was proven to be bounded by $3 / 4$ [72].

\section{Conclusion and outlook}

In this paper, we have illustrated the basic physical and fluid mechanical framework necessary to understand fluidbased locomotion on small scales. After an overview of the fundamentals of biological swimming motility and low Reynolds number hydrodynamics, we have reviewed classical theoretical work on both the external hydrodynamics of organisms - with an emphasis on swimming kinematics — and their various internal actuation mechanisms. We have then outlined areas of current research, including hydrodynamic interactions, locomotion in complex fluids, the design of synthetic swimmers and the search for the optimal swimmer. All along this review, we have attempted to focus on the simple physical picture, and to emphasize how swimming provides a unifying theme for problems arising in disparate fields.

Since new experimental methods promise to reveal the mechanisms for biological locomotion with ever more quantitative detail, future work in the field is likely to be significant. We believe, in particular, that there is great opportunity for theorists, since-as we have emphasized throughout the review-simple calculations are usually sufficient to gain fundamental insight into the mechanisms of locomotion. Detailed numerical computations will also play a crucial role, since many of the issues we discussed, such as hydrodynamic interactions in dense suspensions of swimmers and locomotion in complex fluids, involve nonlinear processes. A further challenge will be to integrate the understanding of basic mechanisms across multiple scales, from the levels of molecular motors to individual cells to large populations of cells. Finally, many of the implications of the ideas sketched in this review have yet to be realized in important areas such as the ecology of marine bacteria, the formation of bacterial biofilms and the mechanics of reproduction.

\section{Acknowledgments}

The authors are pleased to acknowledge the many useful discussions on locomotion at low Reynolds number that they have had over the years with their colleagues, as well as the many people who have given them useful comments on this review: Denis Bartolo, Howard Berg, Alison Berke, Michael Berns, Jacy Bird, Kenny Breuer, Steve Childress, Willow DiLuzio, Henry Fu, David Gagnon, Ray Goldstein, David Gonzalez-Rodriguez, Alexander Grosberg, Peko Hosoi, Greg Huber, Frank Jülicher, Sunghwan Jung, John Kessler, MinJun Kim, MunJu Kim, Stephan Koehler, Marco Cosentino Lagomarsino, Marcos, Sébastien Michelin, Thibaud Normand, Will Ryu, Vivek Shenoy, Mike Shelley, Linda Shi, Antonio de Simone, Saverio Spagnolie, Todd Squires, Srikanth Srigiriraju, Roman Stocker, Howard Stone, John Toner, Renaud Trouilloud, Linda Turner, Annemarie Van Parys, George Whitesides, Chris Wiggins, Charles Wolgemuth and Tony Yu.

They are indebted to Howard Berg, Steve King, Willow DiLuzio, Brian Chan, Peko Hosoi, Marco Cosentino Lagomarsino, Remy Dreyfus, Ramin Golestanian and Kenny Breuer for supplying figures they have used in this review. 
This work was supported in part by the National Science Foundation Grants Nos CTS-0624830 (EL), CBET-0746285 (EL), NIRT-0404031 (TRP) and DMS-0615919 (TRP). The authors thank the Aspen Center for Physics, where this work was conceived.

\section{References}

[1] Berg H C 2004 E. coli in Motion (New York: Springer)

[2] Suarez S S and Pacey A A 2006 Sperm transport in the female reproductive tract Human Reprod. Update 12 23-37

[3] Childress S 1981 Mechanics of Swimming and Flying (Cambridge: Cambridge University Press)

[4] Ellington C P 1984 The Aerodynamics of Hovering Insect Flight (London: The Royal Society)

[5] Vogel S 1996 Life in Moving Fluids (Princeton, NJ: Princeton University Press)

[6] Alexander D E 2002 Nature's Flyers: Birds, Insects, and the Biomechanics of Flight (Baltimore, MD: The Johns Hopkins University Press)

[7] Dudley R 2002 The Biomechanics of Insect Flight: Form, Function, Evolution (Princeton, NJ: Princeton University Press)

[8] Vogel S 2003 Comparative Biomechanics: Life's Physical World (Princeton, NJ: Princeton University Press)

[9] Ludwig W 1930 Zur theorie der Flimmerbewegung (Dynamik, Nutzeffekt, Energiebilanz) J. Comparative Physiol. A 13 397-504

[10] Gray J 1968 Animal Locomotion (London: Norton)

[11] Lighthill J 1975 Mathematical Biofluiddynamics (Philadelphia, PA: SIAM)

[12] Lighthill J 1976 Flagellar hydrodynamics—The John von Neumann Lecture, 1975 SIAM Rev. 18 161-230

[13] Brennen C and Winet H 1977 Fluid mechanics of propulsion by cilia and flagella Annu. Rev. Fluid Mech. 9 339-98

[14] Purcell E M 1977 Life at low Reynolds number Am. J. Phys. 45 3-11

[15] Yates G T 1986 How microorganisms move through water Am. Sci. 74 358-65

[16] Fauci L J and Dillon R 2006 Biofluidmechanics of reproduction Annu. Rev. Fluid Mech. 38 371-94

[17] Holwill M E J 1966 Physical aspects of flagellar movement Physiol. Rev. 46 696-785

[18] Jahn T L and Votta J J 1972 Locomotion of protozoa Annu. Rev. Fluid Mech. 4 93-116

[19] Blum J J and Hines M 1979 Biophysics of flagellar motility Q. Rev. Biophys. 12 103-180

[20] Berg H C 2000 Motile behavior of bacteria Phys. Today $5324-9$

[21] Bray D 2000 Cell Movements (New York: Garland)

[22] Drescher K, Leptos K C and Goldstein R E 2009 How to track protists in three dimensions Rev. Sci. Instrum. 80014301

[23] Turner L, Ryu W S and Berg H C 2000 Real-time imaging of fluorescent flagellar filaments J. Bacteriol. 182 2793-801

[24] Tadir Y, Wright W H, Vafa O, Ord T, Asch R H and Berns M W 1989 Micromanipulation of sperm by a laser generated optical trap Fertil. Steril. 52 870-73

[25] Tadir Y, Wright W H, Vafa O, Ord T, Asch R H and Berns M W 1990 Force generated by human sperm correlated to velocity and determined using a laser generated optical trap Fertil. Steril. 53 944-7

[26] Konig K, Svaasand L, Liu Y G, Sonek G, Patrizio P, Tadir Y, Berns M W and Tromberg B J 1996 Determination of motility forces of human spermatozoa using an $800 \mathrm{~nm}$ optical trap Cell. Mol. Biol. 42 501-9

[27] McCord R P, Yukich J N and Bernd K K 2005 Analysis of force generation during flagellar assembly through optical trapping of free-swimming Chlamydomonas reinhardtii Cell Mot. Cytoskeleton 61 137-44

[28] Chattopadhyay S, Moldovan R, Yeung C and Wu X L 2006 Swimming efficiency of bacterium Escherichia coli Proc. Natl Acad. Sci. USA 103 13712-7

[29] Teff Z, Priel Z and Gheber L A 2007 Forces applied by cilia measured on explants from mucociliary tissue Biophys. $J$. 92 1813-23

[30] Taylor G I 1951 Analysis of the swimming of microscopic organisms Proc. R. Soc. A 209 447-61

[31] Hancock G J 1953 The self-propulsion of microscopic organisms through liquids Proc. R. Soc. Lond. A 217 96-121

[32] Gray J and Hancock G J 1955 The propulsion of sea-urchin spermatozoa J. Exp. Biol. 32 802-14

[33] Stevens J M, Galyov E E and Stevens M P 2006 Actin-dependent movement of bacterial pathogens Nature Rev. Microbiol. 4 91-101

[34] Walsby A E 1994 Gas vesicles Microbiol. Rev. 58 94-144

[35] Berg H C and Anderson R A 1973 Bacteria swim by rotating their flagellar filaments Nature 245 380-2

[36] Berg H C 2009 Bacterial flagellar motor Curr. Biol. 18 R689-91

[37] Berg H C 2003 The rotary motor of bacterial flagella Annu. Rev. Biochem. 72 19-54

[38] Low G, Meister M and Berg H C 1987 Rapid rotation of flagellar bundles in swimming bacteria Nature $325637-40$

[39] Koyasu S and Shirakihara Y 1984 Caulobacter crescentus flagellar filament has a right-handed helical form $\mathrm{J}$. Mol. Biol. 173 125-30

[40] Armitage J P and Schmitt R 1997 Bacterial chemotaxis: Rhodobacter sphaeroides and Sinorhizobium meliloti-variations on a theme? Microbiology 143 3671-82

[41] Marwan W, Alam M and Oesterhelt D 1991 Rotation and switching of the flagellar motor assembly in Halobacterium halobium J. Bacteriol. 173 1971-7

[42] Jarrell K F and McBride M J 2008 The surprisingly diverse ways that prokaryotes move Nature Rev. Microbiol. 6 466-76

[43] Canale-Parola E 1984 The spirochetes Bergey's Manual of Systematic Bacteriology ed N R Krieg and J G Holt (Baltimore, MD: Williams and Wilkins) pp 38-70

[44] Berg H C 1976 How spirochetes may swim J. Theor. Biol. 56 269-73, 56

[45] Goldstein S F, Charon N W and Kreiling J A 1994 Borrelia burgdorferi swims with a planar waveform similar to that of eukaryotic flagella Proc. Natl Acad. Sci. USA $913433-7$

[46] Berg H C and Turner L 1979 Movement of microorganisms in viscous environments Nature 278 349-51

[47] Nakamura S, Adachi Y, Goto T and Magariyama Y 2006 Improvement in motion efficiency of the spirochete Brachyspira pilosicoli in viscous environments Biophys. $J$. 90 3019-26

[48] Murphy G E, Matson E G, Leadbetter J R, Berg H C and Jensen G J 2008 Novel ultrastructures of Treponema primitia and their implications for motility Mol. Microbiol. 67 1184-95

[49] Shaevitz J W, Lee J Y and Fletcher D A 2005 Spiroplasma swim by a processive change in body helicity Cell 122941

[50] Berg H C 2002 How spiroplasma might swim J. Bacteriol. $1842063-4$

[51] Trachtenberg S and Gilad R 2001 A bacterial linear motor: cellular and molecular organization of the contractile cytoskeleton of the helical bacterium Spiroplasma melliferum bc3 Mol. Microbiol. 41 827-48

[52] Wolgemuth C W, Igoshin O and Oster G 2003 The motility of mollicutes Biophys. J. 85 828-42 
[53] Werner M and Simmons L W 2008 Insect sperm motility Biol. Rev. 83 191-208

[54] Quicke D L J, Ingram S N, Baillie H S and Gaitens P V 1992 Sperm structure and ultrastructure in Hymenoptera (insecta) Zoologica Scr. 21 381-402

[55] Hatsumi M and Wakahama K-I 1986 The sperm length and the testis length in Drosophila nasuta subgroup Japan. J. Genet. 61 241-4

[56] Joly D, Bressac C and Lachaise D 1995 Disentangling giant sperm Nature 377202

[57] Mitchell D R 2000 Chlamydomonas flagella J. Phycol. 36 261-73

[58] Gibbons I R 1981 Cilia and flagella of eukaryotes J. Cell Biol. 91 107s-24s

[59] Sleigh M A, Blake J R and Liron N 1988 The propulsion of mucus by cilia Am. Rev. Resp. Dis. 137 726-41

[60] Happel J and Brenner H 1965 Low Reynolds Number Hydrodynamics (Englewood Cliffs, NJ: Prentice Hall)

[61] Kim S and Karrila J S 1991 Microhydrodynamics: Principles and Selected Applications (Boston, MA:

Butterworth-Heinemann)

[62] Leal L G 2007 Advanced Transport Phenomena: Fluid Mechanics and Convective Transport Processes (Cambridge: Cambridge University Press)

[63] Hinch E J 1988 Hydrodynamics at low Reynolds number: a brief and elementary introduction Disorder and Mixing (NATO ASI Series E vol 1) ed E Guyon et al (Dordrecht: Kluwer) pp 43-55

[64] Pozrikidis C 2000 Theoretical and Computational Fluid Dynamics (Oxford: Oxford University Press)

[65] Chwang A T and Wu T Y T 1975 Hydromechanics of low-Reynolds-number flow: II. Singularity method for Stokes flows J. Fluid Mech. 67 787-815

[66] Batchelor B K 1967 An Introduction to Fluid Mechanics (Cambridge: Cambridge University Press)

[67] Pozrikidis C 1992 Boundary Integral and Singularity Methods for Linearized Viscous Flow (Cambridge: Cambridge University Press)

[68] Batchelor G K 1970 The stress system in a suspension of force-free particles J. Fluid Mech. 41 545-70

[69] Blake J R 1971 A note on the image system for a Stokeslet in a no-slip boundary Proc. Camb. Phil. Soc. $70303-10$

[70] Blake J R and Chwang A T 1974 Fundamental singularities of viscous-flow: I. Image systems in vicinity of a stationary no-slip boundary J. Eng. Math. 8 23-9

[71] Pedley T J and Kessler J O 1992 Hydrodynamic phenomena in suspensions of swimming microorganisms Annu. Rev. Fluid Mech. 24 313-58

[72] Stone H A and Samuel A D T 1996 Propulsion of microorganisms by surface distortions Phys. Rev. Lett. $774102-4$

[73] Becker L E, Koehler S A and Stone H A 2003 On self-propulsion of micro-machines at low Reynolds number: Purcell's three-link swimmer J. Fluid Mech. 490 15-35

[74] Shapere A and Wilczek F 1987 Self-propulsion at low Reynolds number Phys. Rev. Lett. 58 2051-4

[75] Shapere A and Wilczek F 1989 Geometry of self-propulsion at low Reynolds number J. Fluid Mech. $198557-85$

[76] Shapere A and Wilczek F 1989 Efficiencies of self-propulsion at low Reynolds number J. Fluid Mech. 198 587-99

[77] Yariv E 2006 Self-propulsion in a viscous fluid: arbitrary surface deformations J. Fluid Mech. 550 139-48

[78] Avron J E and Raz O 2008 A geometric theory of swimming: Purcell's swimmer and its symmetrized cousin New J. Phys. 10063016
[79] Trouilloud R, Yu T S, Hosoi A E and Lauga E 2008 Soft swimming: exploiting deformable interfaces for low Reynolds number locomotion Phys. Rev. Lett. 101048102

[80] Lauga E and Bartolo D 2008 No many-scallop theorem: collective locomotion of reciprocal swimmers Phys. Rev. E 78030901

[81] Vogel S 1997 Squirt smugly, scallop! Nature 385 21-2

[82] Najafi A and Golestanian R 2004 Simple swimmer at low Reynolds number: three linked spheres Phys. Rev. E 69062901

[83] Chwang A T and Wu T Y 1971 Helical movement of micro-organisms Proc. R. Soc. Lond. B 178 327-46

[84] Childress S and Dudley R 2004 Transition from ciliary to flapping mode in a swimming mollusc: flapping flight as a bifurcation in $R e_{\omega} J$. Fluid Mech. 498 257-88

[85] Childress S 2004 Forward flapping flight as a bifurcation in the frequency Reynolds number Proc. 2004 Int. Workshop on Mathematical Fluid Dynamics and Applications (Pohang, Korea) ed S-I Sohn et al pp 9-21

[86] Vandenberghe N, Zhang J and Childress S 2004 Symmetry breaking leads to forward flapping flight J. Fluid Mech. $\mathbf{5 0 6} 147-55$

[87] Alben S and Shelley M 2005 Coherent locomotion as an attracting state for a free flapping body Proc. Natl Acad. Sci. USA 102 11163-6

[88] Vandenberghe N, Childress S and Zhang J 2006 On unidirectional flight of a free flapping wing Phys. Fluids 18014102

[89] Lu X Y and Liao Q 2006 Dynamic responses of a two-dimensional flapping foil motion Phys. Fluids 18098104

[90] Lauga E 2007 Continuous breakdown of Purcell's scallop theorem with inertia Phys. Fluids 19061703

[91] Gonzalez-Rodriguez D and Lauga E 2009 Reciprocal locomotion of dense swimmers in Stokes flow J. Phys.: Condens. Matter 21204103

[92] Camalet S, Julicher F and Prost J 1999 Self-organized beating and swimming of internally driven filaments Phys. Rev. Lett. 82 1590-3

[93] Camalet S and Julicher F 2000 Generic aspects of axonemal beating New J. Phys. 2 1-23

[94] Fu H C, Wolgemuth C W and Powers T R 2008 Beating patterns of filaments in viscoelastic fluids Phys. Rev. E 78041913

[95] Taylor G I 1952 The action of waving cylindrical tails in propelling microscopic organisms Proc. R. Soc. A $211225-39$

[96] Lighthill M J 1952 On the squirming motion of nearly spherical deformable bodies through liquids at very small Reynolds numbers Commun. Pure Appl. Math. 5 109-18

[97] Blake J R 1971 A spherical envelope approach to ciliary propulsion J. Fluid Mech. 46 199-208

[98] Blake J R 1971 Self propulsion due to oscillations on the surface of a cylinder at low Reynolds number Bull. Aust. Math. Soc. 5 255-64

[99] Landau L D and Lifshitz E M 1987 Fluid Mechanics 2nd edn (Oxford: Butterworth-Heinemann)

[100] Reynolds A J 1965 The swimming of minute organisms $J$. Fluid Mech. 23 241-60

[101] Tuck E O 1968 A note on a swimming problem J. Fluid Mech. 31 305-8

[102] Childress $S 2008$ Inertial swimming as a singular perturbation Proc. DSCC 2008, ASME 2008 Dynamic Systems and Control Conf. (Ann Arbour, MI, USA) ed A Galip Ulsoy pp DSCC2008-210DSCC2008-210

[103] Yundt A P, Shack W J and Lardner T J 1975 Applicability of hydrodynamic analyses of spermatozoan motion J. Exp. Biol. 62 27-41 
[104] Lauga E 2007 Floppy swimming: viscous locomotion of actuated elastica Phys. Rev. E 75041916

[105] Tam D S-W 2008 Motion at low Reynolds number $P h D$ Thesis Massachusetts Institute of Technology, Cambridge, MA

[106] Magariyama Y, Sugiyama S, Muramoto K, Kawagishi I, Imae Y and Kudo S 1995 Simultaneous measurement of bacterial flagellar rotation rate and swimming speed Biophys. J. 69 2154-62

[107] Purcell E M 1997 The efficiency of propulsion by a rotating flagellum Proc. Natl Acad. Soc., USA 94 11307-11

[108] Li G and Tang J X 2006 Low flagellar motor torque and high swimming efficiency of Caulobacter crescentus swarmer cells Biophys. J. 91 2726-34

[109] Johnson R E and Brokaw C J 1979 Flagellar hydrodynamics - comparison between resistive-force theory and slender-body theory Biophys. J. 25 113-27

[110] Cox R G 1970 The motion of long slender bodies in a viscous fluid: I. General theory J. Fluid Mech. 44 791-810

[111] Batchelor G K 1970 Slender body theory for particles of arbitrary cross section in Stokes flow J. Fluid Mech. 44 419-40

[112] Tillett J P K 1970 Axial and transverse Stokes flow past slender axisymmetric bodies J. Fluid Mech. 44 401-17

[113] Keller J B and Rubinow S I 1976 Slender body theory for slow viscous flow J. Fluid Mech. 75 705-14

[114] Geer J 1976 Stokes flow past a slender body of revolution $J$. Fluid Mech. 78 577-600

[115] Johnson R E 1980 An improved slender body theory for Stokes flow J. Fluid Mech. 99 411-31

[116] Lighthill J 1996 Reinterpreting the basic theorem of flagellar hydrodynamics J. Eng. Math. 30 25-34

[117] Gueron S and Liron N 1992 Ciliary motion modeling and dynamic multicilia interactions Biophys. J. 63 1045-58

[118] Gueron S and Liron N 1993 Simulations of three-dimensional ciliary beats and cilia interactions Biophys. J. 65 499-507

[119] Gueron S, Levit-Gurevich K, Liron N and Blum J J 1997 Cilia internal mechanism and metachronal coordination as the result of hydrodynamical coupling Proc. Natl Acad. Sci. USA 94 6001-6

[120] Gueron S and Levit-Gurevich K 1999 Energetic considerations of ciliary beating and the advantage of metachronal coordination Proc. Natl Acad. Sci. USA 96 12240-5

[121] Cortez R 2001 The method of regularized stokeslets SIAM J. Sci. Comput. 23 1204-25

[122] Cortez R 2008 personal communication

[123] Macnab R M and Aizawa S I 1984 Bacterial motility and the bacterial flagellar motor Annu. Rev. Biophys. Bioeng. 13 51-83

[124] Schuster S C and Khan S 1994 The bacterial flagellar motor Annu. Rev. Biophys. Biomol. Struct. 23 509-39

[125] Matsuura S, Kamiya R and Asakura S 1978 Transformation of straight flagella and recovery of motility in a mutant Escherichia coli J. Mol. Biol. 118 431-40

[126] Wolgemuth C W, Powers T R and Goldstein R E 2000 Twirling and whirling: Viscous dynamics of rotating elastic filaments Phys. Rev. Lett. 84 1623-6

[127] Lim S and Peskin C S 2004 Simulations of the whirling instability by the immersed boundary method SIAM J. Sci. Comput. 25 2066-83

[128] Wada H and Netz R R 2006 Non-equilibrium hydrodynamics of a rotating filament Europhys. Lett. 75 645-51

[129] Kim M J and Powers T R 2005 Deformation of a helical filament by flow and electric or magnetic fields Phys. Rev. E 71021914

[130] Darnton N C, Turner L, Rojevsky S and Berg H C 2007 On torque and tumbling in swimming Escherichia coli $J$. Bacteriol. 189 1756-64
[131] Takano Y, Yoshida K, Kudo S, Nishitoba M and Magariyama Y 2003 Analysis of small deformation of helical flagellum of swimming Vibrio alginolyticus JSME Int. J. C 46 1241-7

[132] Macnab R M and Ornston M K 1977 Normal to curly flagellar transitions and their role in bacterial tumbling-Stabilization of an alternative quaternary structure by mechanical force J. Mol. Biol. 112 1-30

[133] Hotani H 1982 Micro-video study of moving bacterial flagellar filaments iii. cyclic transformation induced by mechanical force J. Mol. Biol. 156791

[134] Goldstein R E, Goriely A, Huber G and Wolgemuth C W 2000 Bistable helices Phys. Rev. Lett. 84 1631-4

[135] Coombs D, Huber G, Kessler J O and Goldstein R E 2002 Periodic chirality transformations propagating on bacterial flagella Phys. Rev. Lett. 89 118102-1-118102-4

[136] Srigiriraju S V and Powers T R 2005 Continuum model for polymorphism of bacterial flagella Phys. Rev. Lett. 94248101

[137] Srigiriraju S V and Powers T R 2006 Model for polymorphic transitions in bacterial flagella Phys. Rev. E 73011902

[138] Machin K E 1958 Wave propagation along flagella. J. Exp. Biol. 35 796-806

[139] Machin K E 1963 The control and synchronization of flagellar movement Proc. R. Soc. B 158 88-104

[140] Wiggins C H and Goldstein R E 1998 Flexive and propulsive dynamics of elastica at low Reynolds number Phys. Rev Lett. 80 3879-82

[141] Lowe C P 2003 Dynamics of filaments: modelling the dynamics of driven microfilaments Phil. Trans. R. Soc. Lond., Ser. B 358 1543-50

[142] Lagomarsino M C, Capuani F and Lowe C P 2003 A simulation study of the dynamics of a driven filament in an Aristotelian fluid J. Theor. Biol. 224 215-24

[143] Yu T S, Lauga E and Hosoi A E 2006 Experimental investigations of elastic tail propulsion at low Reynolds number Phys. Fluids 18091701

[144] Kosa G, Shoham M and Zaaroor M 2007 Propulsion method for swimming microrobots IEEE Trans. Robot. 23 137-50

[145] Riveline D, Wiggins C H, Goldstein R E and Ott A 1997 Elastohydrodynamic study of actin filaments using fluorescence microscopy Phys. Rev. E 56 R1330-3

[146] Wiggins C H, Riveline D, Ott A and Goldstein R E 1998 Trapping and wiggling: elastohydrodynamics of driven microfilaments Biophys. J. 74 1043-60

[147] Landau L D and Lifshitz E M 1986 Theory of Elasticity 3rd edn (Oxford: Pergamon)

[148] Klapper I 1996 Biological applications of the dynamics of twisted elastic rods J. Comput. Phys. $125325-37$

[149] Goldstein R E, Powers T R and Wiggins C H 1998 Viscous nonlinear dynamics of twist and writhe Phys. Rev. Lett. 80 5232-5

[150] Manghi M, Schlagberger X and Netz R R 2006 Propulsion with a rotating elastic nanorod Phys. Rev. Lett. 96068101

[151] Qian B, Powers T R and Breuer K S 2008 Shape transition and propulsive force of an elastic rod rotating in a viscous fluid Phys. Rev. Lett. 1078101

[152] Coq N, du Roure O, Marthelot J, Bartolo D and Fermigier M 2008 Rotational dynamics of a soft filament: wrapping transition and propulsive forces Phys. Fluids. 20051703

[153] Nonaka S, Tanaka Y, Okada Y, Takeda S, Harada A, Kanai Y, Kido M and Hirokawa N 1998 Randomization of left-right asymmetry due to loss of nodal cilia generating leftward flow of extraembryonic fluid in mice lacking KIF3B motor protein Cell 95 829-37

[154] Hilfinger A and Jülicher F 2008 The chirality of ciliary beats Phys. Biol. $5016003+12$ 
[155] Satir P 1968 Studies on cilia: III. Further studies on the cilium tip and a 'sliding filament' model of ciliary motility J. Cell Biol. 39 77-94

[156] Summers K E and Gibbons I R 1971 Adenosine triphosphate-induced sliding of tubules in trypsin-treated flagella of sea-urchin sperm Proc. Natl Acad. Sci. USA $683092-6$

[157] Brokaw C J 1972 Flagellar movement: a sliding filament model Science 178 455-62

[158] Brokaw C J 1989 Direct measurements of sliding between outer doublet microtubules in swimming sperm flagella Science 243 1593-6

[159] Riedel-Kruse I H, Hilfinger A, Howard J and Jülicher F 2007 How molecular motors shape the flagellar beat HFSP J. 1 192-208

[160] Fu H C, Powers T R and Wolgemuth H C 2007 Theory of swimming filaments in viscoelastic media Phys. Rev. Lett. $99258101-5$

[161] Gibbons B H and Gibbons I R 1972 Flagellar movement and adenosine triphosphatase activity in sea urchin sperm extracted with triton X-100 J. Cell Biol. 54 75-97

[162] Brokaw C J 1975 Molecular mechanism for oscillation in flagella and muscle Proc. Natl Acad. Sci. USA 72 3102-6

[163] Jülicher F and Prost J 1997 Spontaneous oscillations of collective molecular motors Phys. Rev. Lett. 78 4510-3

[164] Murase M, Hines M and Blum J J 1989 Properties of an excitable dynein model for bend propagation in cilia and flagella J. Theor. Biol. 139 413-30

[165] Lindemann C 1994 A geometric clutch hypothesis to explain oscillations of the axoneme of cilia and flagella $J$. Theor. Biol. 168 175-89

[166] Lindemann C 1994 A model of flagellar and ciliary functioning which uses the forces transverse to the axoneme as the regulator of dynein activation Cell Motil. Cytoskel. 29 141-54

[167] Lindemann C 2002 Geometric clutch model version: III. The role of the inner and outer arm dyneins in the ciliary beat Cell Motil. Cytoskel. 52 242-54

[168] Brokaw C 2002 Computer simulation of flagellar movement: VIII. Coordination of dynein by local curvature control can generate helical bending waves Cell Motil. Cytoskel. 53 103-24

[169] Wada H and Netz R R 2007 Model for self-propulsive helical filaments: kink-pair propagation Phys. Rev. Lett. 99108102

[170] Charon N W, Daughtry G R, McCuskey R S and Franz G N 1984 Microcinematographic analysis of tethered Leptospira illini J. Bacteriol. 160 1067-73

[171] Goldstein S and Charon N 1990 Multiple-exposure photographic analysis of a motile spirochete Proc. Natl Acad. Sci. USA 87 4895-9

[172] Jung S, Mareck K, Fauci L and Shelley M J 2007 Rotational dynamics of a superhelix towed in a Stokes fluid Phys. Fluids 19103105

[173] Kessler J and Wojciechowski M F 1997 Collective behavior and dynamics of swimming bacteria Bacteria as Multicellular Organisms ed J A Shapiro and M Dworkin (Oxford: Oxford University Press) pp 417-50

[174] Mendelson N H, Bourque A, Wilkening K, Anderson K R and Watkins J C 1999 Organized cell swimming motions in Bacillus subtilis colonies: patterns of short-lived whirls and jets J. Bacteriol. 181 600-9

[175] Kessler J 2000 Dynamics of swimming bacteria at low and high volume fraction Proc. Int. Conf. on Differential Equations (Berlin, Germany, 1-7 August 1999) ed B Fiedler et al (Singapore: World Scientific) pp 1284-7

[176] Wu X L and Libchaber A 2000 Particle diffusion in a quasi-two-dimensional bacterial bath Phys. Rev. Lett. 84 3017-20
[177] Dombrowski C, Cisneros L, Chatkaew S, Goldstein R E and Kessler J O 2004 Self-concentration and large-scale coherence in bacterial dynamics Phys. Rev. Lett. 93098103

[178] Tuval I, Cisneros L, Dombrowski C, Wolgemuth C W, Kessler J and Goldstein R E 2005 Bacterial swimming and oxygen transport near contact lines Proc. Natl Acad. Sci. USA 102 2277-82

[179] Cisneros L H, Cortez R, Dombrowski C, Goldstein R E and Kessler J O 2007 Fluid dynamics of self-propelled micro-organisms, from individuals to concentrated populations Exp. Fluids $\mathbf{4 3} 737-53$

[180] Sokolov A, Aranson I S, Kessler J O and Goldstein R E 2007 Concentration dependence of the collective dynamics of swimming bacteria Phys. Rev. Lett. 98158102

[181] Wolgemuth C W 2008 Collective swimming and the dynamics of bacterial turbulence Biophys. $J$. 95 1564-74

[182] Moore H, Dvoráková K, Jenkins N and Breed W 2002 Exceptional sperm cooperation in the wood mouse Nature 418 174-7

[183] Moore H D M and Taggart D A 1995 Sperm pairing in the opossum increases the efficiency of sperm movement in a viscous environment Biol. Reprod. 52 947-53

[184] Hayashi F 1998 Sperm co-operation in the Fishfly Parachauliodes japonicus Funct. Ecol. 12 347-50

[185] Riedel I H, Kruse K and Howard J 2005 A self-organized vortex array of hydrodynamically entrained sperm cells Science 309 300-3

[186] Drescher K, Leptos K C, Tuval I, Ishikawa T, Pedley T J and Goldstein R E 2009 Dancing Volvox hydrodynamic bound states of swimming algae Phys. Rev. Lett. 102168101

[187] Dreyfus R, Baudry J and Stone H A 2005 Purcell's 'rotator': mechanical rotation at low Reynolds number Eur. Phys. $J$. B 47 161-4

[188] Guell D C, Brenner H, Frankel R B and Hartman H 1988 Hydrodynamic forces and band formation in swimming magnetotactic bacteria J. Theor. Biol. 135 525-42

[189] Pooley C M, Alexander G P and Yeomans J M 2007 Hydrodynamic interaction between two swimmers at low Reynolds number Phys. Rev. Lett. 99228103

[190] Liao Q, Subramanian G, DeLisa M P, Koch D L and Wu M M 2007 Pair velocity correlations among swimming Escherichia coli bacteria are determined by force-quadrupole hydrodynamic interactions Phys. Fluids 19061701

[191] Ishikawa T, Simmonds M P and Pedley T J 2006 Hydrodynamic interaction of two swimming model micro-organisms J. Fluid Mech. 568 119-60

[192] Ishikawa T and Hota M 2006 Interaction of two swimming Paramecia J. Exp. Biol. 209 4452-63

[193] Ishikawa T, Sekiya G, Imai Y and Yamaguchi T 2007 Hydrodynamic interaction between two swimming bacteria Biophys. J. 93 2217-25

[194] Simha R A and Ramaswamy S 2002 Hydrodynamic fluctuations and instabilities in ordered suspensions of self-propelled particles Phys. Rev. Lett. 89058101

[195] Saintillan D and Shelley M J 2007 Orientational order and instabilities in suspensions of self-locomoting rods Phys. Rev. Lett. 99058102

[196] Saintillan D and Shelley M J 2008 Instabilities and pattern formation in active particle suspensions: kinetic theory and continuum simulations Phys. Rev. Lett. 100178103

[197] Ishikawa T and Pedley T J 2008 Coherent structures in monolayers of swimming particles Phys. Rev. Lett. 1 088103

[198] Hernandez J P-Ortiz, Stoltz C G and Graham M D 2005 Transport and collective dynamics in suspensions of confined swimming particles Phys. Rev. Lett. 95204501 
[199] Ishikawa T and Pedley T J 2007 Diffusion of swimming model micro-organisms in a semi-dilute suspension $J$. Fluid Mech. $\mathbf{5 8 8}$ 437-62

[200] Mehandia V and Nott P R 2008 The collective dynamics of self-propelled particles J. Fluid Mech. 595 239-64

[201] Underhill P T, Hernandez J P-Ortiz and Graham M D 2008 Diffusion and spatial correlations in suspensions of swimming particles Phys. Rev. Lett. 100248101

[202] Hatwalne Y, Ramaswamy S, Rao M and Simha R A 2004 Rheology of active-particle suspensions Phys. Rev. Lett. 92118101

[203] Ishikawa T and Pedley T J 2007 The rheology of a semi-dilute suspension of swimming model micro-organisms J. Fluid Mech. 588 399-435

[204] Berke A P, Turner L, Berg H C and Lauga E 2008 Hydrodynamic attraction of swimming microorganisms by surfaces Phys. Rev. Lett. 101038102

[205] Oldroyd J G 1950 On the formulation of rheological equations of state Proc. R. Soc. A 200 523-41

[206] Bird R B 1976 Useful non-Newtonian models Annu. Rev. Fluid Mech. 8 13-34

[207] Bird R B, Armstrong R C and Hassager O 1987 Dynamics of Polymeric Liquids vol 1, 2nd edn Fluid Mechanics (New York: Wiley-Interscience)

[208] Bird R B, Curtiss C F, Armstrong R C and Hassager O 1987 Dynamics of Polymeric Liquids vol 2, 2nd edn Kinetic Theory (New York: Wiley-Interscience)

[209] Tanner R I 1988 Engineering Rheology 2nd edn (Oxford: Clarendon)

[210] Larson R G 1988 Constitutive Equations for Polymer Melts and Solutions (Boston, MA: Butterworth-Heinemann)

[211] Doi M and Edwards S F 1988 The Theory of Polymer Dynamics (Oxford: Oxford University Press)

[212] Bird R B and Wiest J M 1995 Constitutive equations for polymeric liquids Annu. Rev. Fluid Mech. 27 169-93

[213] Larson R G 1999 The Structure and Rheology of Complex Fluids (Oxford: Oxford University Press)

[214] Fletcher M and Marshall K C 1982 Are solid surfaces of ecological significance to aquatic bacteria? Adv. Microb. Ecol. 6 199-230

[215] Cooper G L, Schiller A L and Hopkins C C 1988 Possible role of capillary action in pathogenesis of experimental catheter-associated dermal tunnel infections J. Clin. Microbiol. 26 8-12

[216] Harkes G, Dankert J and Feijen J 1992 Bacterial migration along solid surfaces Appl. Environ. Microbiol. 58 1500-5

[217] Costerton J W, Lewandowski Z, Caldwell D E, Korber D R and Lappinscott H M 1995 Microbial biofilms Annu. Rev. Microbiol. 49 711-45

[218] Vanloosdrecht M C M, Lyklema J, Norde W and Zehnder A J B 1990 Influence of interfaces on microbial activity Microbiol. Rev. 54 75-87

[219] Harshey R M 2003 Bacterial motility on a surface: many ways to a common goal Annu. Rev. Microbiol. 57 249-73

[220] Katz D F 1974 Propulsion of microorganisms near solid boundaries J. Fluid Mech. 64 33-49

[221] Katz D F, Blake J R and Paverifontana S L 1975 Movement of slender bodies near plane boundaries at low Reynolds number J. Fluid Mech. 72 529-40

[222] Katz D F and Blake J R 1975 Flagellar motions near walls 1975 Swimming and Flying in Nature vol 1 ed T Y Wu et al (New York: Plenum) pp 173-84

[223] Fauci L J and Mcdonald A 1995 Sperm motility in the presence of boundaries Bull. Math. Biol. 57 679-99

[224] Lauga E, DiLuzio W R, Whitesides G M and Stone H A 2006 Swimming in circles: motion of bacteria near solid boundaries Biophys. J. 90 400-12
[225] Ramia M, Tullock D L and Phan-Thien N 1993 The role of hydrodynamic interaction in the locomotion of microorganisms Biophys. J. 65 755-78

[226] Frymier P D, Ford R M, Berg H C and Cummings P T 1995 Three-dimensional tracking of motile bacteria near a solid planar surface Proc. Natl Acad. Sci. USA 92 6195-9

[227] Frymier P D and Ford R M 1997 Analysis of bacterial swimming speed approaching a solid-liquid interface AIChE J. 43 1341-7

[228] Vigeant M A S and Ford R M 1997 Interactions between motile Escherichia coli and glass in media with various ionic strengths, as observed with a three-dimensional tracking microscope Appl. Environ. Microbiol. 63 3474-9

[229] DiLuzio W R, Turner L, Mayer M, Garstecki P, Weibel D B, Berg H C and Whitesides G M 2005 Escherichia coli swim on the right-hand side Nature 435 1271-4

[230] Rothschild L 1963 Non-random distribution of bull spermatozoa in a drop of sperm suspension Nature 198 1221-2

[231] Winet H, Bernstein G S and Head J 1984 Spermatozoon tendency to accumulate at walls is strongest mechanical response J. Androl. 519

[232] Winet H, Bernstein G S and Head J 1984 Observations on the response of human spermatozoa to gravity, boundaries and fluid shear J. Reprod. Fertil. 70 511-23

[233] Cosson J, Huitorel P and Gagnon C 2003 How spermatozoa come to be confined to surfaces Cell Motil. Cytoskel. $5456-63$

[234] Woolley D M 2003 Motility of spermatozoa at surfaces Reproduction 126 259-70

[235] Galajda P, Keymer J E, Chaikin P and Austin R H 2007 A wall of funnels concentrates swimming bacteria $J$. Bacteriol. 189 8704-7

[236] Elgeti J and Gompper G 2009 Self-propelled rods near surfaces Europhys. Lett. 8538002

[237] Fauci L J 1990 Interaction of oscillating filaments-a computational study J. Comput. Phys. 86 294-313

[238] Elfring G and Lauga E 2009 Hydrodynamic phase-locking of swimming microorganisms Phys. Rev. Lett. 103088101

[239] Strogatz S 2000 From Kuramoto to Crawford: exploring the onset of synchronization in populations of coupled oscillators Physica D 143 1-20

[240] Blake J R and Sleigh M A 1974 Mechanics of ciliary locomotion Biol. Rev. Camb. Phil. Soc. 49 85-125

[241] Salathe M 2007 Regulation of mammalian ciliary beating Annu. Rev. Physiol. 69 401-22

[242] Smith D J, Blake J R and Gaffney E A 2008 Fluid mechanics of nodal flow due to embryonic primary cilia J. R. Soc. Interface 5 567-73

[243] Lenz P and Ryskin A 2006 Collective effects in ciliar arrays Phys. Biol. 3 285-94

[244] Vilfan A and Julicher F 2006 Hydrodynamic flow patterns and synchronization of beating cilia Phys. Rev. Lett. 96058102

[245] Guirao B and Joanny J F 2007 Spontaneous creation of macroscopic flow and metachronal waves in an array of cilia Biophys. J. 92 1900-17

[246] Niedermayer T, Eckhardt B and Lenz P 2008 Synchronization, phase locking and metachronal wave formation in ciliary chains Chaos $\mathbf{1 8} 037128$

[247] Qian B, Jiang H, Gagnon D A, Powers T R and Breuer K S 2009 Minimal model for hydrodynamic synchronization http://arxiv.org/abs/0904.2347

[248] Anderson R A 1975 Formation of the bacterial flagellar bundle Swimming and Flying in Nature vol 1 ed T Y-T Wu et al (New York: Plenum)

[249] Macnab R M 1977 Bacterial flagella rotating in bundles: a study in helical geometry Proc. Natl Acad. Sci. USA 74 221-5 
[250] Powers T R 2002 Role of body rotation in bacterial flagellar bundling Phys. Rev. E 65040903

[251] Kim M, Bird J C, Van Parys A J, Breuer K S and Powers T R 2003 A macroscopic scale model of bacterial flagellar bundling Proc. Natl Acad. Sci. USA 100 15481-5

[252] Kim M J, Kim M M J, Bird J C, Park J, Powers T R and Breuer K S 2004 Particle image velocimetry experiments on a macro-scale model for bacterial flagellar bundling Exp. Fluids 37 782-8

[253] Flores H, Lobaton E, Mendez-Diez S, Tlupova S and Cortez R 2005 A study of bacterial flagellar bundling Bull. Math. Biol. 67 137-68

[254] Kim M and Powers T R 2004 Hydrodynamic interactions between rotating helices Phys. Rev. E 69061910

[255] Reichert M and Stark H 2005 Synchronization of rotating helices by hydrodynamic interactions Eur. Phys. J. E 17 493-500

[256] Block S M, Blair D F and Berg H C 1989 Compliance of bacterial flagella measured with optical tweezers Nature 338 514-8

[257] Block S M, Blair D F and Berg H C 1991 Compliance of bacterial polyhooks measured with optical tweezers Cytometry 12 492-6

[258] Bigelow J L, Dunson D B, Stanford J B, Ecochard R, Gnoth C and Colombo B 2004 Mucus observations in the fertile window: a better predictor of conception then timing of intercourse Human Reprod. 19 889-92

[259] Wolf D P, Blasco L, Khan M A and Litt M 1977 Human cervical mucus: I. Rheologic characteristics Fertil. Steril. 28 41-6

[260] Wolf D P, Blasco L, Khan M A and Litt M 1977 Human cervical mucus: II. Changes in viscoelasticity during ovulatory menstrual cycle Fertil. Steril. 28 47-52

[261] Wolf D P, Sokoloski J, Khan M A and Litt M 1977 Human cervical mucus: III. Isolation and characterization of rheologically active mucin Fertil. Steril. 28 53-8

[262] Wolf D P, Blasco L, Khan M A and Litt M 1978 Human cervical mucus: IV. Viscoelasticity and sperm penetrability during ovulatory menstrual-cycle Fertil. Steril. 30 163-9

[263] Katz D F, Mills R N and Pritchett T R 1978 Movement of human spermatozoa in cervical mucus J. Reprod. Fertil. 53 259-65

[264] Dunn P F and Picologlou B F 1976 Viscoelastic properties of cumulus oophorus Biorheology 13 379-84

[265] Suarez S S, Katz D F, Owen D H, Andrew J B and Powell R L 1991 Evidence for the function of hyperactivated motility in sperm Biol. Reprod. $44375-81$

[266] Suarez S S and Dai X B 1992 Hyperactivation enhances mouse sperm capacity for penetrating viscoelastic media Biol. Reprod. 46 686-91

[267] Suarez S S 1996 Hyperactivated motility in sperm J. Androl. $17331-5$

[268] Ho H C and Suarez S S 2001 Hyperactivation of mammalian spermatozoa: function and regulation Reproduction 122 519-26

[269] Suarez S S and Ho H C 2003 Hyperactivated motility in sperm Reprod. Dom. Anim. 38 119-24

[270] Hwang S H, Litt M and Forsman W C 1969 Rheological properties of mucus Rheol. Acta 8 438-48

[271] Tam P Y, Katz D F and Berger S A 1980 Nonlinear viscoelastic properties of cervical mucus Biorheology 17 465-78

[272] Eliezer N 1974 Viscoelastic properties of mucus Biorheology 11 61-8

[273] Litt M, Khan M A and Wolf D P 1976 Mucus rheology-relation to structure and function Biorheology $1337-48$
[274] Lauga E 2007 Propulsion in a viscoelastic fluid Phys. Fluids 19083104

[275] Montecucco C and Rappuoli R 2001 Living dangerously: how helicobacter pylori survives in the human stomach Nature Rev. Mol. Cell Biol. 2457

[276] Wolgemuth C W, Charon N W, Goldstein S F and Goldstein R E 2006 The flagellar cytoskeleton of the spirochetes J. Mol. Microbiol. Biotechnol. 11221

[277] Chaudhury T K 1979 Swimming in a viscoelastic liquid $J$. Fluid Mech. 95 189-97

[278] Fulford G R, Katz D F and Powell R L 1998 Swimming of spermatozoa in a linear viscoelastic fluid Biorheology 35 295-309

[279] Ross S M and Corrsin S 1974 Results of an analytical mode of mucociliary pumping J. Appl. Physiol. 37 333-40

[280] Fu H C, Wolgemuth C W and Powers T R 2009 Swimming speeds of filaments in nonlinearly viscoelastic fluids Phys. Fluids 21033102

[281] Lauga E 2009 Life at high Deborah number Europhys. Lett. 8664001

[282] Normand T and Lauga E 2008 Flapping motion and force generation in a viscoelastic fluid Phys. Rev. E 78061907

[283] Ishijima S, Oshio S and Mohri H 1986 Flagellar movement of human spermatozoa Gamete Res. 13 185-97

[284] Teran J, Fauci L and Shelley M 2008 Peristaltic pumping and irreversibility of a Stokesian viscoelastic fluid Phys. Fluids 20073101

[285] Leoni M, Kotar J, Bassetti B, Cicuta P and Cosentino Lagomarsino M 2009 A basic swimmer at low Reynolds number Soft Matter 5 472-6

[286] Tam D and Hosoi A E 2007 Optimal stroke patterns for Purcell's three-link swimmer Phys. Rev. Lett. 98068105

[287] Najafi A and Golestanian R 2005 Propulsion at low Reynolds number J. Phys.: Condens. Matter 17 S1203-8

[288] Golestanian R and Ajdari A 2008 Analytic results for the three-sphere swimmer at low Reynolds number Phys. Rev. E 77036308

[289] Golestanian R 2008 Three-sphere low-Reynolds-number swimmer with a cargo container Eur. Phys. J. E 25 1-4

[290] Golestanian R and Ajdari A 2008 Mechanical response of a small swimmer driven by conformational transitions Phys. Rev. Lett. 1038101

[291] Felderhof B U 2006 The swimming of animalcules Phys. Fluids 18063101

[292] Avron J E, Kenneth O and Oaknin D H 2005 Pushmepullyou: an efficient micro-swimmer New J. Phys. 7234

[293] Ogrin F Y, Petrov P G and Winlove C P 2008 Ferromagnetic microswimmers Phys. Rev. Lett. 100218102

[294] Koiller J, Ehlers K and Montgomery R 1996 Problems and progress in microswimming J. Nonlinear Sci. 6 507-41

[295] Alexander G P and Yeomans J M 2008 Dumb-bell swimmers Eur. Phys. Lett. 8334006

[296] Dreyfus R, Baudry J, Roper M L, Fermigier M, Stone H A and Bibette J 2005 Microscopic artificial swimmers Nature 437 862-5

[297] Yu T S, Lauga E and Hosoi A E Swimming using elastic tail propulsion, unpublished

[298] Cebers A 2005 Flexible magnetic filaments Curr. Opin. Colloid Interface Sci. 10 167-75

[299] Gauger E and Stark H 2006 Numerical study of a microscopic artificial swimmer Phys. Rev. E 74021907

[300] Roper M, Dreyfus R, Baudry J, Fermigier M, Bibette J and Stone H A 2006 On the dynamics of magnetically driven elastic filaments J. Fluid Mech. 554 167-90

[301] Keaveny E E and Maxey M R 2008 Spiral swimming of an artificial micro-swimmer J. Fluid Mechanics 598 293-319

[302] Roper M, Dreyfus R, Baudry J, Fermigier M, Bibette J and 
Stone H A 2008 Do magnetic micro-swimmers move like eukaryotic cells? Proc. R. Soc. A 464 877-904

[303] Thaokar R M, Schiessel H and Kulic I M 2007 Hydrodynamics of a rotating torus Eur. Phys. J. B 60 325-36

[304] Thaokara R M 2008 Hydrodynamic interaction between two rotating tori Eur. Phys. J. B $6147-58$

[305] Leshansky A M and Kenneth O 2008 Surface tank treading: propulsion of purcell's toroidal swimmer Phys. Fluids 20063104

[306] Leshansky A M, Kenneth O, Gat O and Avron J E 2007 A frictionless microswimmer New J. Phys. 9126

[307] Paxton W F, Kistler K C, Olmeda C C, Sen A, St Angelo S K, Cao Y Y, Mallouk T E, Lammert P E and Crespi V H 2004 Catalytic nanomotors: autonomous movement of striped nanorods J. Am. Chem. Soc. 126 13424-31

[308] Fournier-Bidoz S, Arsenault A C, Manners I and Ozin G A 2005 Synthetic self-propelled nanorotors Chem. Commun. $441-3$

[309] Kline T R, Paxton W F, Mallouk T E and Sen A 2005 Catalytic nanomotors: remote-controlled autonomous movement of striped metallic nanorods Angew. Chem. Int. Edit. 44 744-6

[310] Mano N and Heller A 2005 Bioelectrochemical propulsion $J$. Am. Chem. Soc. 127 11574-5

[311] Paxton W F, Sundararajan S, Mallouk T E and Sen A 2006 Chemical locomotion Angew. Chem. Int. Edit. 45 5420-9

[312] Ruckner G and Kapral R 2007 Chemically powered nanodimers Phys. Rev. Lett. 98150603

[313] Howse J R, Jones R A L, Ryan A J, Gough T, Vafabakhsh R and Golestanian R 2007 Self-motile colloidal particles: from directed propulsion to random walk Phys. Rev. Lett. 99048102

[314] Tao Y G and Kapral R 2008 Design of chemically propelled nanodimer motors J. Chem. Phys. 128164518

[315] Golestanian R, Liverpool T B and Ajdari A 2005 Propulsion of a molecular machine by asymmetric distribution of reaction products Phys. Rev. Lett. 94220801

[316] Golestanian R, Liverpool T B and Ajdari A 2007 Designing phoretic micro- and nano-swimmers New J. Phys. 9126

[317] Cordova-Figueroa U M and Brady J F 2008 Osmotic propulsion: the osmotic motor Phys. Rev. Lett. 100158303

[318] Jülicher F and Prost J 2009 Generic theory of colloidal transport Eur. Phys. J. E 28 27-36
[319] Anderson J 1989 Colloidal transport by interfacial forces Annu. Rev. Fluid Mech. 2161

[320] Kim M J and Breuer K S 2004 Enhanced diffusion due to motile bacteria Phys. Fluids 16 L78-L81

[321] Darnton N, Turner L, Breuer K and Berg H C 2004 Moving fluid with bacterial carpets Biophys. J. 86 1863-70

[322] Weibel D B, Garstecki P, Ryan D, Diluzio W R, Mayer M, Seto J E and Whitesides G M 2005 Microoxen: microorganisms to move microscale loads Proc. Natl Acad. Soc. USA $10211963-7$

[323] Raz O and Leshansky A M 2008 Efficiency of cargo towing by a microswimmer Phys. Rev. E 77055305

[324] Raz O and Avron J E 2007 Swimming, pumping and gliding at low Reynolds numbers New J. Phys. 9437

[325] Kim Y W and Netz R R 2006 Pumping fluids with periodically beating grafted elastic filaments Phys. Rev. Lett. 96158101

[326] Tabata O, Hirasawa H, Aoki S, Yoshida R and Kokufuta E 2002 Ciliary motion actuator using self-oscillating gel Sensors Actuators A 95 234-8

[327] Pironneau O and Katz D F 1974 Optimal swimming of flagellated microorganisms J. Fluid Mech. 66 391-415

[328] Pironneau O and Katz D F 1975 Optimal swimming of motion of flagella Swimming and Flying in Nature vol 1 ed T Y Wu et al (New York: Plenum) pp 161-72

[329] Higdon J J L 1979 Hydrodynamic analysis of flagellar propulsion J. Fluid Mech. 90 685-711

[330] Higdon J J L 1979 Hydrodynamics of flagellar propulsion-helical waves J. Fluid Mech. 94 331-51

[331] Lighthill J 1996 Helical distributions of stokeslets. J. Eng. Math. 30 35-78

[332] Fujita T and Kawai T 2001 Optimum shape of a flagellated microorganism JSME Int. J. Ser. C 44 952-7

[333] Higdon J J L 1979 The generation of feeding currents by flagellar motions J. Fluid Mech. 94305

[334] Alouges F, DeSimone A and Lefebvre A 2008 Optimal strokes for low Reynolds number swimmers: an example J. Nonlinear Sci. 18 277-302

[335] Alouges F, DeSimone A and Lefebvre A 2009 Optimal strokes for axisymmetric microswimmers Eur. Phys. J. E 28 279-84

[336] Avron J E, Gat O and Kenneth O 2004 Optimal swimming at low Reynolds numbers Phys. Rev. Lett. 93186001 